\title{
Synthesis of Colloidal Nanocrystal Heterostructures for High- Efficiency Light Emission
}

Yifei Lu

Follow this and additional works at: https://researchrepository.wvu.edu/etd

\section{Recommended Citation}

Lu, Yifei, "Synthesis of Colloidal Nanocrystal Heterostructures for High-Efficiency Light Emission" (2014). Graduate Theses, Dissertations, and Problem Reports. 6116.

https://researchrepository.wvu.edu/etd/6116

This Thesis is protected by copyright and/or related rights. It has been brought to you by the The Research Repository @ WVU with permission from the rights-holder(s). You are free to use this Thesis in any way that is permitted by the copyright and related rights legislation that applies to your use. For other uses you must obtain permission from the rights-holder(s) directly, unless additional rights are indicated by a Creative Commons license in the record and/ or on the work itself. This Thesis has been accepted for inclusion in WVU Graduate Theses, Dissertations, and Problem Reports collection by an authorized administrator of The Research Repository @ WVU. For more information, please contact researchrepository@mail.wvu.edu. 


\title{
Synthesis of Colloidal Nanocrystal Heterostructures for High-Efficiency Light Emission
}

\author{
by \\ Yifei Lu
}

Thesis submitted to the Benjamin M. Statler College of Engineering and Mineral Resources at West Virginia University in partial fulfillment of the requirements

for the Master degree

In

Electrical Engineering

Committee Members

Xian-An Cao, Ph.D., Committee Chairperson

Parviz Famouri, Ph.D.

Mark A. Jerabek, Ph.D.

Lane Department of Computer Science and Electrical Engineering

Morgantown, West Virginia 2014

Keywords: Quantum Dot; Light-Emitting Diode;

Copyright 2014 Yifei Lu 


\section{Abstract \\ Synthesis of Colloidal Nanocrystal Heterostructures for High-Efficiency Light \\ Emission}

By Yifei Lu

Group II-VI semiconductor nanocrystals, particularly those based on $\mathrm{ZnCdS}(\mathrm{Se})$, can be synthesized using well established chemical colloidal processes, and have been a subject of extensive research over the past decade. Their optical properties can be easily tuned through size and composition variations, making them very attractive for many optoelectronic applications including light-emitting diodes (LEDs) and solar cells. Incorporation of diverse internal heterostructures provides an additional means for tuning the optical and electronic properties of conventional $\mathrm{ZnCdS}(\mathrm{Se})$ nanocrystals. Extensive bandgap and strain engineering may be applied to the resultant nanocrystal heterostructures to achieve desirable properties and enhanced performance. Despite the high scientific and practical interests of this unique class of nanomaterials, limited efforts have been made to explore their synthesis and potential device applications.

This thesis focuses on the synthesis, engineering, characterization, and device demonstration of two types of CdSe-based nanocrystal heterostructures: core/multishell quantum dots (QDs) and QD quantum wells (QDQWs). Their optical properties have been tuned by bandgap and strain engineering to achieve efficient photoluminescence (PL) and electroluminescence (EL). Firstly, yellow light-emitting CdSe QDs with a strain-compensated $\mathrm{ZnS} / \mathrm{ZnCdS}$ bilayer shell were synthesized using the successive ion layer adsorption and reaction technique and the effects of the shell on the luminescent properties were investigated. The core/shell/shell QDs enjoyed the benefits of excellent exciton confinement by the $\mathrm{ZnS}$ intermediate shell and strain compensation by the $\mathrm{ZnCdS}$ outer shell, and exhibited $40 \%$ stronger PL and a smaller peak redshift upon shell growth compared to conventional $\mathrm{CdSe} / \mathrm{ZnCdS} / \mathrm{ZnS}$ core/shell/shell QDs with an intermediate lattice adaptor. $\mathrm{CdSe} / \mathrm{ZnS} / \mathrm{ZnCdS}$ QD-LEDs had a luminance of $558 \mathrm{~cd} / \mathrm{m}^{2}$ at $20 \mathrm{~mA} / \mathrm{cm}^{2}$, $28 \%$ higher than that of $\mathrm{CdSe} / \mathrm{ZnCdS} / \mathrm{ZnS}$ QD-LEDs.

Secondly, CdS/CdSe/ZnS QDQWs were synthesized and their luminescence was tuned in an effort to realize efficient blue light emission from CdSe nanocrystals. CdSe QWs with a well width of $1.05 \mathrm{~nm}$ emitted at $467 \mathrm{~nm}$ with a spectral full-width-at-half-maximum of $\sim 30 \mathrm{~nm}$. With a 3-monolayer $\mathrm{ZnS}$ cladding layer which also acts as a passivating and strain-compensating layer, the QDQWs acquired a $\sim 35 \%$ PL quantum yield (QY). Blue and green EL was obtained from QDQW-LEDs with 3-4.5 monolayers (MLs) QWs. It was found that as the well width and peak wavelength decreased, the overall EL was increasingly dominated by defect state emission, suggesting the device performance is mainly limited by poor charge injection into the QDQWs. 


\section{ACKNOWLEDGEMENTS}

First, I would like to thank my advisor, Prof. Xian-An Cao for his guidance, patience, support during the past three years and providing me the opportunity to work in this interesting research topic of quantum dot (QD) materials and QD-based optoelectronic devices. Also, I would like to thank Prof. Parviz Famouri and Prof. Mark A. Jerabek for their availability as my committee members and their invaluable suggestions.

Moreover, I want to thank Dr. Kolin Brown, Dr. Weiqiang Ding, Harley Hart of WVU Shared Research Facility (SRF) for all the technical and non-technical trainings and suggestions.

My special gratitude goes to my lab mates, Dr. Yiqiang Zhang and Rajeev Acharya, who introduced me to the handling and working of the equipments, and most recently Xiaomong $\mathrm{Li}$ for the discussions, lab assistance and support. I will also take this chance to thank fellow lab mates for providing a pleasant work environment in the shared research facilities.

Finally, I would like to thank my wife and parents, whose constant belief, love and support has given me all the strength and self-motivation; and to whom I dedicate this thesis. 


\section{TABLE OF CONTENTS}

\begin{tabular}{ll|l} 
ABSTRACT & II
\end{tabular}

ACKNOWLEDGEMENTS III

TABLE OF CONTENTS IV

CHAPTER 1 INTRODUCTION

1.1 Colloidal Nanocrystal (NC) QDs 1

1.1.1 Quantum Confinement Effects 2

1.1.2 Properties of Colloidal QDs 4

$\begin{array}{ll}1.2 \text { Nanocrystal Heterostructures } & 6\end{array}$

1.2.1 Colloidal Core/Shell (CS) QDs $\quad 7$

$\begin{array}{ll}\text { 1.2.2 Multiple Shell QDs } & 10\end{array}$

1.2.3 QD/QW NC Heterostructures $\quad 11$

$\begin{array}{ll}1.3 \text { Device Application of Colloidal QDs } & 13\end{array}$

1.3.1 QD-based LEDs 13

1.3.2 QD-based Solar Cells $\quad 18$

1.3.3 Challenges Facing QD-based Devices $\quad 20$

1.4 Research Focuses 21

CHAPTER 2 SYNTHESIS OF CDSE QUANTUM DOTS WITH STRAIN COMPENSATED

MULTISHELL FOR HIGH-EFFICIENCY LIGHT EMISSION 22

2.1 Synthesis of CdSe QDs with strain compensated multishell 22

2.1.1 Synthesis of CdSe Core QDs 22

2.1.2 Growth of Core/Shell (CS) QDs 24

2.1.3 Growth of Core/Shell/Shell (CSS) QDs 28

2.2 Demonstration of High-Efficiency LEDs based on strain-compensated QDs 34

2.2.1 Structural Optimization 34

2.2.2 Fabrication and Characterization of CSS QD-LEDs 37

2.3 Conclusions 41

CHAPTER 3 SYNTHESIS OF CDSE QUANTUM-DOT QUANTUM-WELL NANOCRYSTALS

FOR BLUE LIGHT EMISSION

3.1 Synthesis and Characterization of CdSe Quantum-Dot Quantum-Well (QDQW) nanocrysals (NCs) 43

3.1.1 Synthesis of CdS Core QDs $\quad 43$

3.1.2 Growth of QDQW Structure 46

3.2 Demonstration of Blue-Emitting QDQW-LEDs 51

$\begin{array}{ll}3.3 \text { Conclusions } & 57\end{array}$

CHAPTER 4 CONCLUSIONS AND FUTURE WORK

$\begin{array}{ll}4.1 \text { Conclusions } & 58\end{array}$

$\begin{array}{ll}4.2 \text { Future Work } & 59\end{array}$

APPENDIX

REFERENCES 


\section{Chapter 1 Introduction}

Nanostructured materials are of great interests to scientists these days, as they link the bulk and molecular levels, leading to a wide range of applications, especially in electronics, optoelectronics and biology. Among different nanomaterials, quantum dots (QDs) attract a great deal of attention due to their unique optical properties, such as size dependent emission wavelength, narrow emission peak and broad excitation range. Optoelectronic devices based on QDs, including light-emitting diodes (LEDs), solar cells, and sensitive photodetectors, with their high efficiency, low power consumption, and easy manufacturing, offer a good solution for various energy-conversion technologies, especially energy saving and harvesting.

\subsection{Colloidal Nanocrystal (NC) QDs}

Colloidal quantum dots (CQDs) are nanometer-sized crystalline semiconductor particles formulated by hundreds to thousands of atoms synthesized using colloidal chemical processes. The most extensively used technique is the hot injection method. ${ }^{[1]}$ Usually, the size of CQDs ranges from $1 \mathrm{~nm}$ to $20 \mathrm{~nm}$ in diameter. As synthesized CQDs are suspended in the solution phase in most situations, such nanoparticles have attracted great attention as an important new class of materials. The ever growing interest in these materials derives from the convenient fabrication of solids directly from the solution-phase, as well as their rationally-engineered, wide-ranging spectral tunability afforded by the quantum size effect. ${ }^{[2,3]}$ Figure 1.1 (a) and (b) showed the TEM images of arrays of $\sim 5 \mathrm{~nm}$ diameter red CdSe CQDs and blue CdS CQDs. ${ }^{[4]}$ As seen, the QDs are self-assembled into close-packed and ordered arrays with good monodispersity, demonstrating a narrow size distribution. Figure 1.1

(c) is a typical schematic view of the structure of core/shell QDs in the colloidal solution, whose surface is capped with long chain organic ligands. Due to the outer 
capping layer, the QDs are well defined and form a uniform distribution. Colloidal semiconductor QDs under intensive research include those based on group II-VI, IV-VI and III-V compound semiconductors.

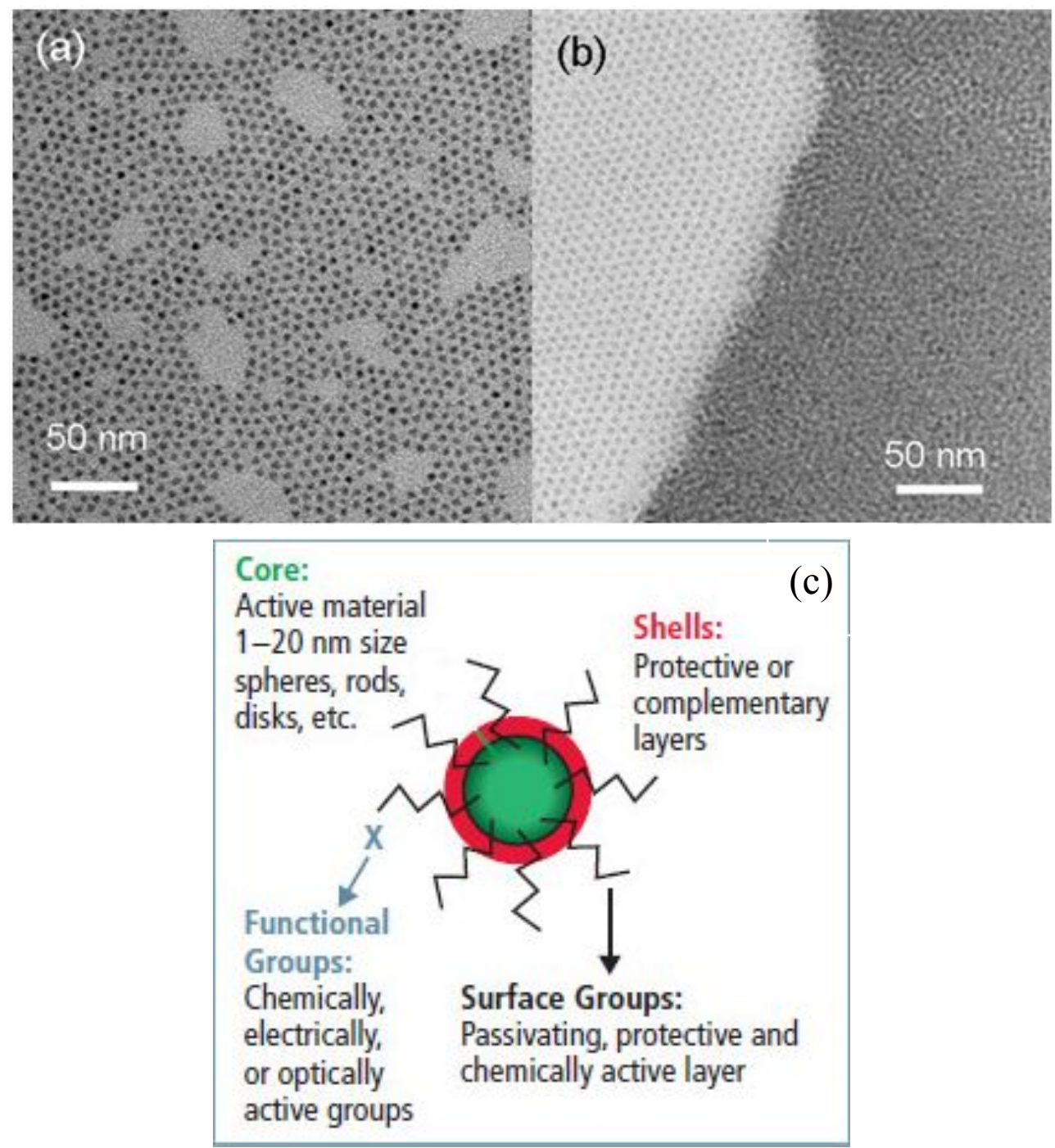

Fig. 1.1 Transmission electron microscopic image of samples of (a) CdSe QDs; (b) CdS QDs (c) Colloidal QDs composition (Courtesy of the Bawendi Group, MIT)

\subsubsection{Quantum Confinement Effects}

A semiconductor is a material which has electrical conductivity to a degree between that of a metal and an insulator. The so-called band gap between the conduction and valence bands, determines the emitting light wavelength by its width. In bulk materials, the width is a fixed parameter determined by the material itself. 
However, in the case of nanoscale semiconductor materials, the size is comparable with the spatial extent of the electronic wave function. As charge carriers are spatially confined, and the material dimensions are smaller than their Bohr exciton radius, their energy is quantized. This is called the quantum confinement effect, which may occur in 0-2 D nanostructures.

The quantum confinement effect in semiconductors was first seen in the 1970 's ${ }^{[5]}$, when molecular beam epitaxy was developed as a thin film growing technique of semiconductor materials at atomic precision thickness, known as quantum wells (QWs). Such structures are available to tune the energy of band-to-band transitions by adjusting the well thickness, and thus many optical properties can be tuned. Moreover, the one-dimension confinement leads to enhanced recombination rates of electrons and holes. These two advantages of QWs make them well suited for many optoelectronic applications. Studies of similar effects have been extended to lower-dimension nanostructures including quantum wires and QDs, which have even stronger quantum confinement and associated unique properties. Figure 1.2 illustrates the energy levels in different structures, from single atom, to QDs, to bulk material, as well as the associated DOS modified by the quantum confinement effects. $^{[6]}$

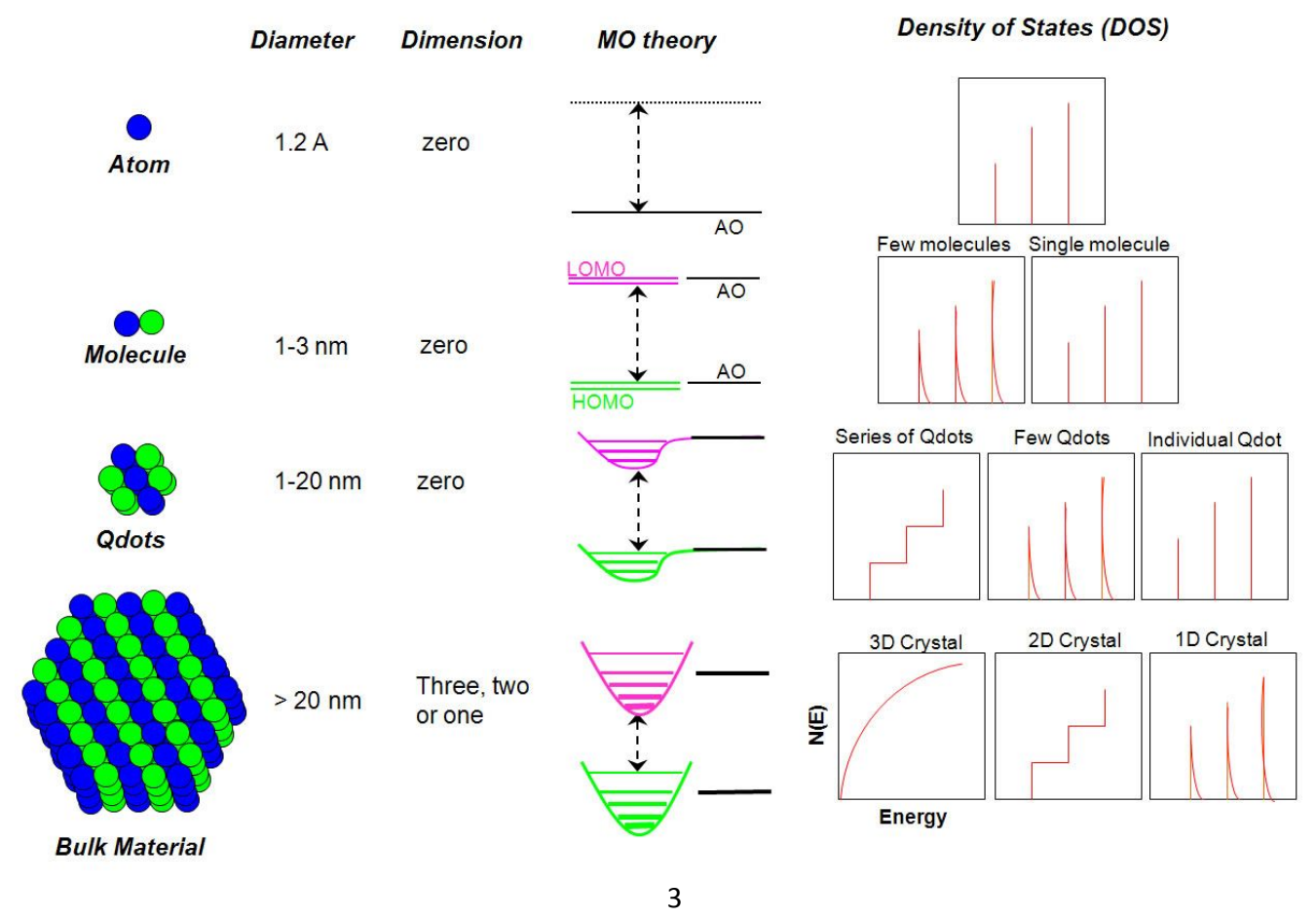


Fig. 1.2 Schematic illustration of the changes of the density of quantum states (DOS) with changes in the number of atoms in materials (MO: molecular orbital; HOMO: highest occupied MO; LUMO: lowest unoccupied MO; AO: atomic orbital)

\subsubsection{Properties of Colloidal QDs}

Charge confinement inside a tiny particle like a QD leads to a collapse of the continuous energy bands of bulk materials into discrete energy states (Figure 1.3). Within the simple "quantum box" model (Efros and Efros 1982), the size-dependent contribution to the energy gap is simply proportional to $1 / \mathrm{R}^{2}$ for a spherical QD with radius $\mathrm{R}$, indicating that the energy gap increases as the QD size decreases. This model directly explains that, within the quantum confinement regime, the optical and electronic properties of QDs are strongly dependent on their size. ${ }^{[7]}$

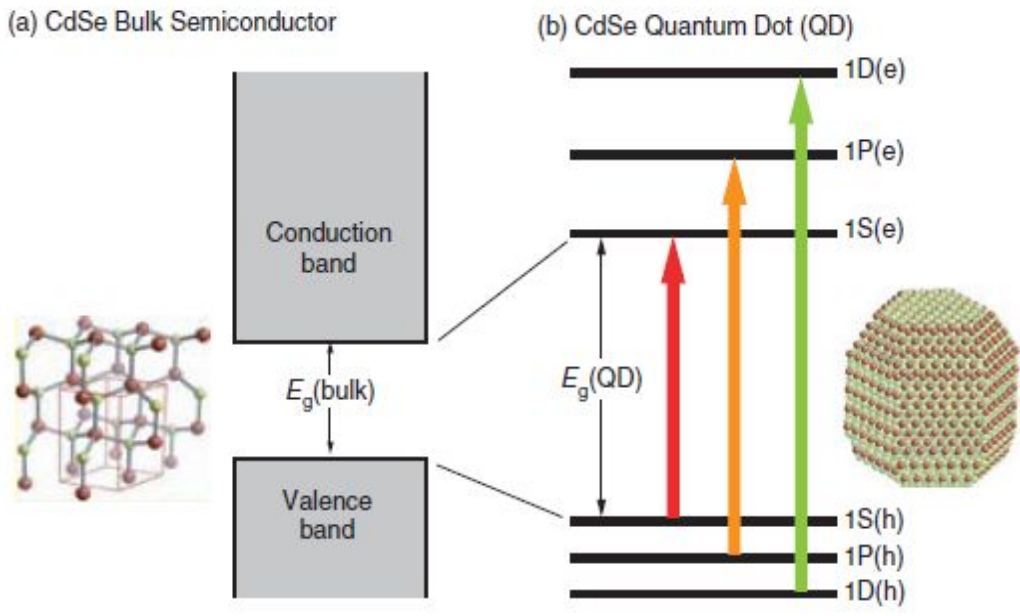

(c)

$$
\begin{gathered}
E_{\mathrm{g}}(\mathrm{QD})=E_{\mathrm{g} 0}+\frac{\hbar^{2} \pi^{2}}{2 m_{\mathrm{eh}} R^{2}} \\
m_{\mathrm{eh}}=\frac{m_{\mathrm{e}} m_{\mathrm{h}}}{m_{\mathrm{e}}+m_{\mathrm{h}}}
\end{gathered}
$$

$m_{e}=$ effective electron mass

$m_{\mathrm{h}}=$ effective hole mass

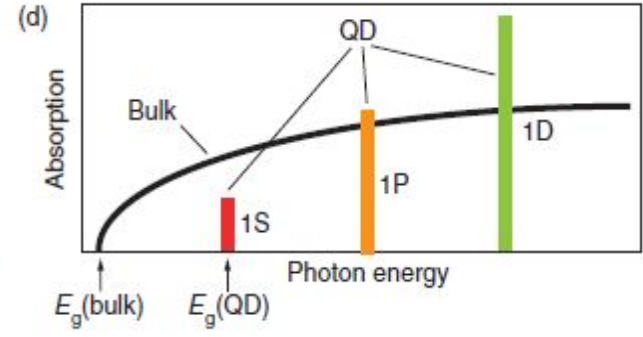

Fig. 1.3 (a) A bulk semiconductor such as CdSe has continuous conduction and valence energy bands separated by a "fixed" energy gap, Eg(bulk). Electrons normally occupy all states up to the edge of the valence band, whereas states in the conduction band are empty. (b) A QD is characterized by discrete atomic-like states with energies that are determined by the QD radius R. These well-separated QD 
states can be labeled with atomic-like notations, such as 1S, 1P, and 1D. (c) The expression for the size-dependent separation between the lowest electron and hole QD states-Eg(QD), the QD energy gap-was obtained with the spherical "quantum box" model. (d) This schematic represents the continuous absorption spectrum of a bulk semiconductor (black line) compared with a discrete absorption spectrum of a QD (colored bars). ${ }^{[7]}$

Among all of the properties of colloidal QDs, the most exciting one is their size-dependent optical property as a result of strong quantum confinement (Figure 1.4). As the size of QDs is reduced, the electronic excitations shift to higher energy, and the absorption and photoluminescence (PL) spectra display blue shifts. By changing the Colloidal CdSe QDs size, emitting light could be tuned to span the entire visible spectrum. As shown in the top plot of Figure 1.4, the CdSe QDs of different sizes emit light with a color ranging from blue to red. Also, the PL spectra of the CdSe QDs in the bottom show a narrow $(\sim 30 \mathrm{~nm})$ full width at half maximum (FWHM), which suggests all the QDs in an ensemble are highly uniform in size and shape, leading to color-saturated PL. The size-dependent, color-saturated emission of QDs across the entire visible spectrum is unsurpassed by any class of organic chromospheres, making them very attractive for both biological imaging ${ }^{[8]}$ and light emission $^{[9]}$.
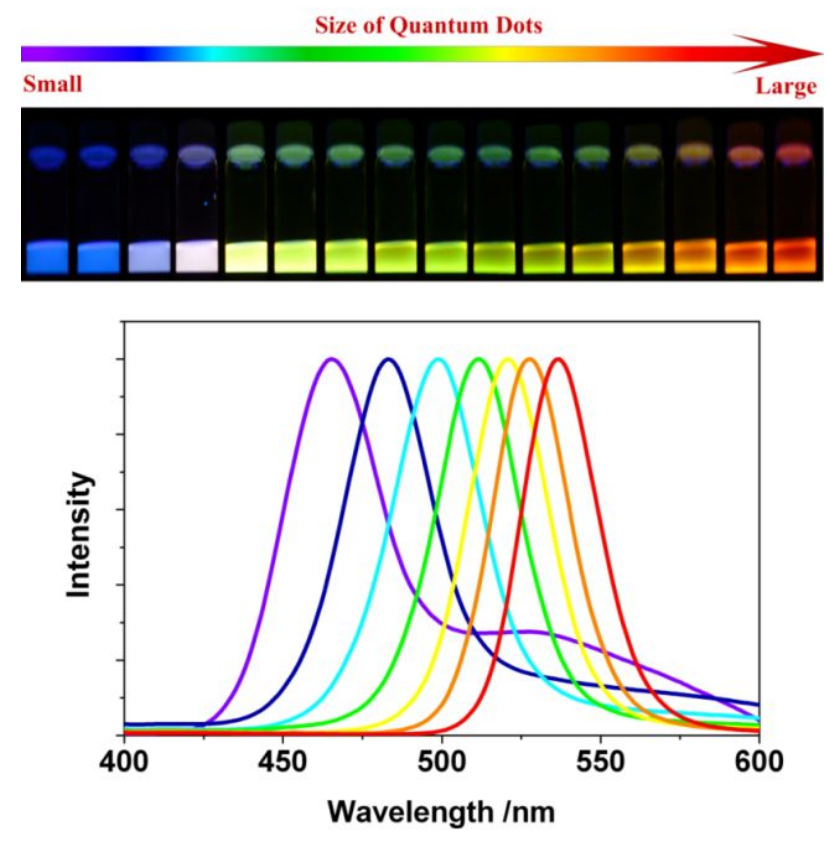
Fig. 1.4 Top: Sixteen emission colors from small (blue) to large (red) CdSe QDs excited by a near-ultraviolet lamp; size of QDs can be from $\sim 1 \mathrm{~nm}$ to $\sim 10 \mathrm{~nm}$ (depends on several parameters, see text for details). Bottom: Photoluminescence spectra of some of the CdSe QDs. ${ }^{[6]}$

Generally, monodipersed QDs are developed by introducing organic molecules that adsorb on the QD surface and act as capping agents, in a colloidal solution. Alternatively, QDs may be grown epitaxially on solid substrates. Many epitaxial QDs have been fabricated with a wide range of shapes and sizes by high-vacuum deposition techniques, such as molecular beam epitaxy or metallorganic vapor phase epitaxy, and directly buried into optoelectronic devices. Fabrication of expitaxial QDs requires complicated and expensive technological processes. In contrast, colloidal QDs with uniform size and shape can be synthesized using low-cost colloidal chemical processes, offering opportunities for inexpensive device fabrication on versatile substrates by solution processing such as spin coating, dip coating, or inkjet printing. Colloidal QDs are thus suitable for low-cost and large-area manufacturing of optoelectronic devices. ${ }^{[10]}$

\subsection{Nanocrystal (NC) Heterostructures}

Due to the high surface-to-volume ratio of QDs, electronic quantum states associated with the surface (called surface states) have significant effects on the optical properties of QDs. In general, surface states arise from unsatisfied bonds at the reconstructed surface, and may be affected by nonstoichiometry and voids. Therefore, they can trap charge carriers (electron or hole) and behave as recombination centers. They may also act as the reducing (electrons) or oxidizing (holes) agents. These electronic behaviors and electrochemical reactions at the surface can significantly affect the overall electronic and optical properties of QDs. As a result, surface states have a significant impact on the performance of optoelectronic devices based on QDs. Surface passivation of QDs is thus critical. A common strategy is to passivate the QD surface with a thin shell of a wider band gap semiconductor, forming a NC heterostructure called core/shell (CS) QDs. ${ }^{[1]}$ The shell reduces the number of surface 
dangling bonds and physically separates the optically active core from its surrounding medium. Consequently, CS QDs have much improved luminescence quantum yield (QY) and stability against photo-oxidation as compared to QD cores. However, such a shell layer may act as either an insulator or a barrier for the conduction of charge. ${ }^{[6]}$ Like CS QDs, many other types of NC heterostructures may be synthesized by the colloidal processes. Their layer structure can be easily tailored and energy bands can be engineered to achieve desirable optical properties. ${ }^{[12,13]}$

\subsubsection{Colloidal Core/Shell (CS) QDs}

An effective strategy to passivate the QDs surface is the use of inorganic layers, particularly a semiconductor material with a larger band-gap, forming a core/shell structure. In this manner, the fluorescence efficiency and stability against photo-oxidation of various types of QDs has seen significant improvement. Furthermore, by the appropriate choice of the core and shell materials, it is possible to tune the emission wavelength in a larger spectral window than with either the materials alone. ${ }^{[11]}$ After the pioneering work in the 1980 s and the development of powerful chemical synthesis routes at the end of the $1990 \mathrm{~s},{ }^{[14-16]}$ a strongly increasing number of articles have been devoted to CS QDs in the past several years.

Depending on the bandgaps and the relative position of electronic energy levels of the involved semiconductors, the core/shell NC heterostructures could be divided into three distinguished types, denominated type-I, reverse type-I, and type- II band alignment (Figure 1.5). For Type-I, the bandgap of the shell material is larger than that of the core and both electrons and holes are confined in the core. In reverse Type-I CS QDs, the bandgap of the shell material is smaller than that of the core and, depending of the thickness of the shell, the holes and electrons are partially or completely confined in the shell. In Type-II heterostructures, either the valence-band edge or the conduction band edge of the shell material is located in the bandgap of the core. Upon excitation of the QDs, the staggered band alignment leads to a spatial separation of electrons and holes in different regions of the CS structure. ${ }^{[11]}$ 
The shell shows quite different roles in these three types of CS structures. In type-I CS QDs, the shell is used to passivate the surface of the core with the goal to improve its optical properties. In reverse type-I systems, a material with narrower bandgap is overgrown onto the wider bandgap core, where charge carriers are at least partially delocalized in the shell and the emission wavelength can be tuned by the shell's thickness. In type-II systems, shell growth aims at a significant red shift of the emission wavelength of the QDs and in some cases, more efficient separation of photogenerated electron-hole pairs. The interest of these systems is the possibility to manipulate the shell thickness and thereby tune the optical properties, which are difficult to attain with conventional materials and structures.
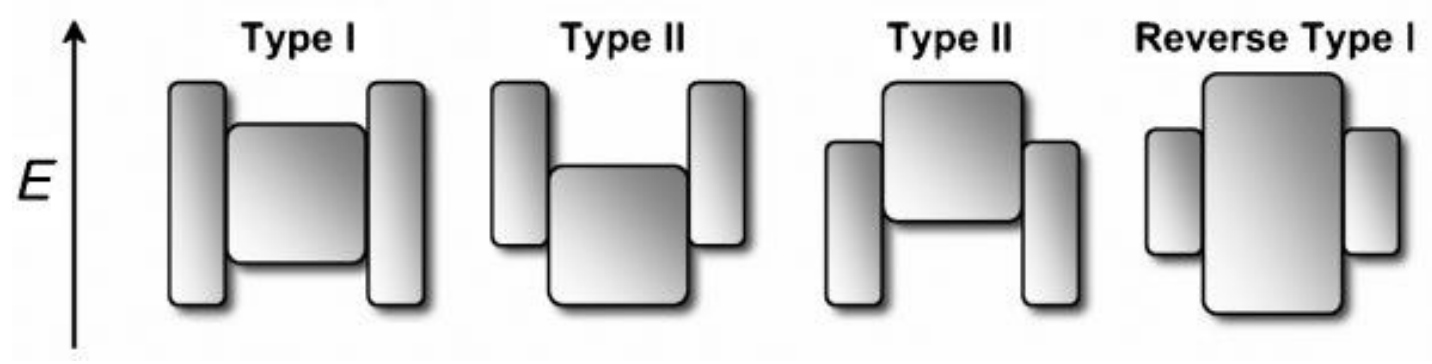

Fig. 1.5 Schematic representation of the energy-level alignment in different core/shell systems realized with semiconductor QDs to date. The upper and lower edges of the rectangles correspond to the positions of the conduction- and valence-band edge of the core (center) and shell materials, respectively..$^{[11]}$

The general requirement for the synthesis of CS QDs with satisfactory optical properties includes two parts: appropriate band alignment and crystalline match. In the opposite case, growth of the shell results in strain and the formation of defect states, which of course, will act as trap states for photogenerated charge carriers and decrease the fluorescence QY. ${ }^{[17]}$ Synthesis of CS colloidal QDs usually includes two steps: (i) initial core QD synthesis followed by purification, and (ii) the subsequent shell growth and purification. The setup and process are shown in Figure 1.6. The growth temperature is usually lower than the nucleation temperature of the core, in order to prevent the nucleation of the shell material and uncontrolled ripening of the core QDs. Such a process will eliminate the unreacted precursors and side products 
before the shell growth, in comparison of the so-called one-pot approach process without an intermediate purification step.

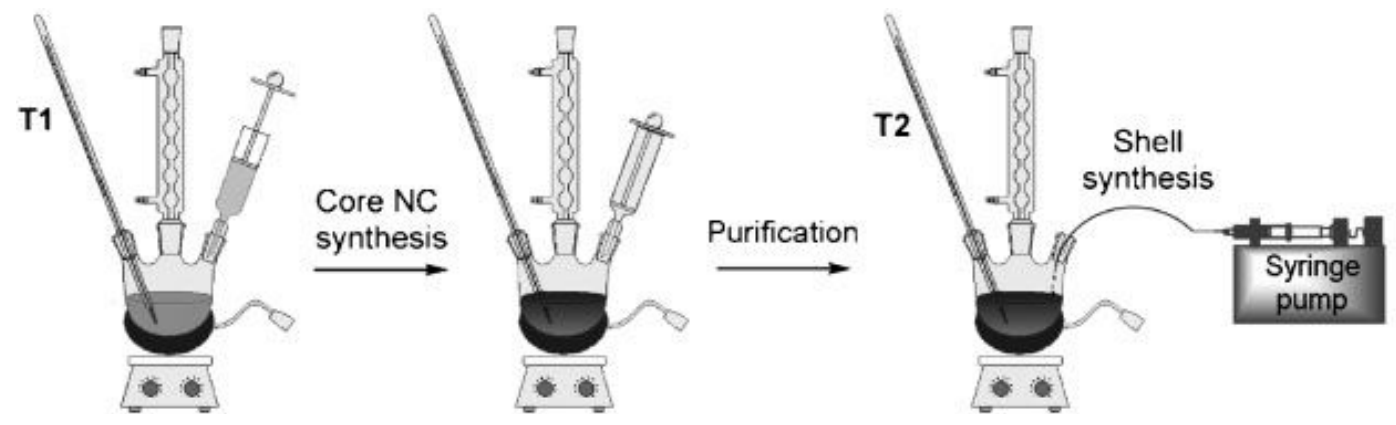

Fig.1.6 Two-step synthesis of core/shell QDs. ${ }^{[1]}$

A unique process called SILAR (successive ion layer adsorption and reaction) was recently developed for shell growth, ${ }^{[18]}$ which can result in monodisperse CS QDs with improved uniformity. In this process, only one monolayer is formed at a time by alternative injection of cationic and anionic precursors with appropriate quantity. The synthesis of CdSe/CdS CS QDs using SILAR is schematically illustrated in Figure 1.7.

\section{SILAR technique}

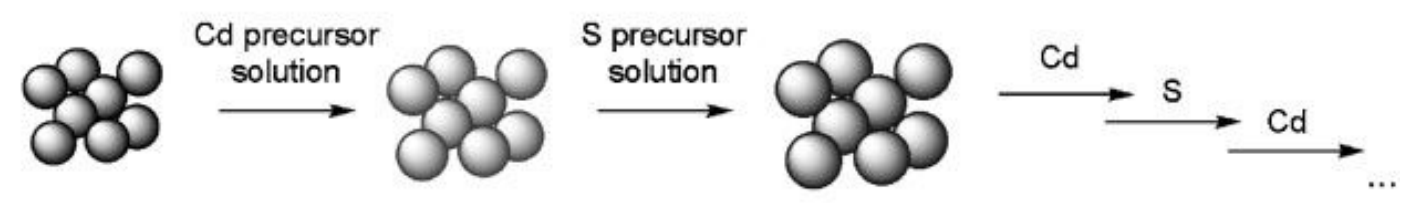

\section{CdSe core NCs}

Fig.1.7 Shell synthesis using the SILAR method, schematically illustrated for the CdSe/CdS core/shell system. ${ }^{[11]}$

A CdSe-based system exhibiting a different band alignment is $\mathrm{CdSe} / \mathrm{CdS}$. In this common cation heterostructure, a large band offset for the holes is combined with a relatively small one for the electrons. Epitaxial growth is favored by the comparably small lattice mismatch of around $4 \%$ between the core and shell material. Peng et al. reported the synthesis and detailed characterization of a series of CdSe/CdS QDs with core diameters ranging from 2.3 to $3.9 \mathrm{~nm}$ and QYs above $50 \% .^{[16]}$ While in this early report organometallic precursors (dimethylcadmium, bis(trimethylsilyl)sulfide) was 
used, the synthesis of this CS system was more recently carried out using air-stable precursors, i.e. cadmium oleate and elemental sulfur dissolved in octadecene. ${ }^{[18]}$ Figure 1.18 shows the TEM images and optical characterization of $\mathrm{CdSe} / \mathrm{CdS} \mathrm{CS}$ QDs with 1-5 monolayers of CdS shell.
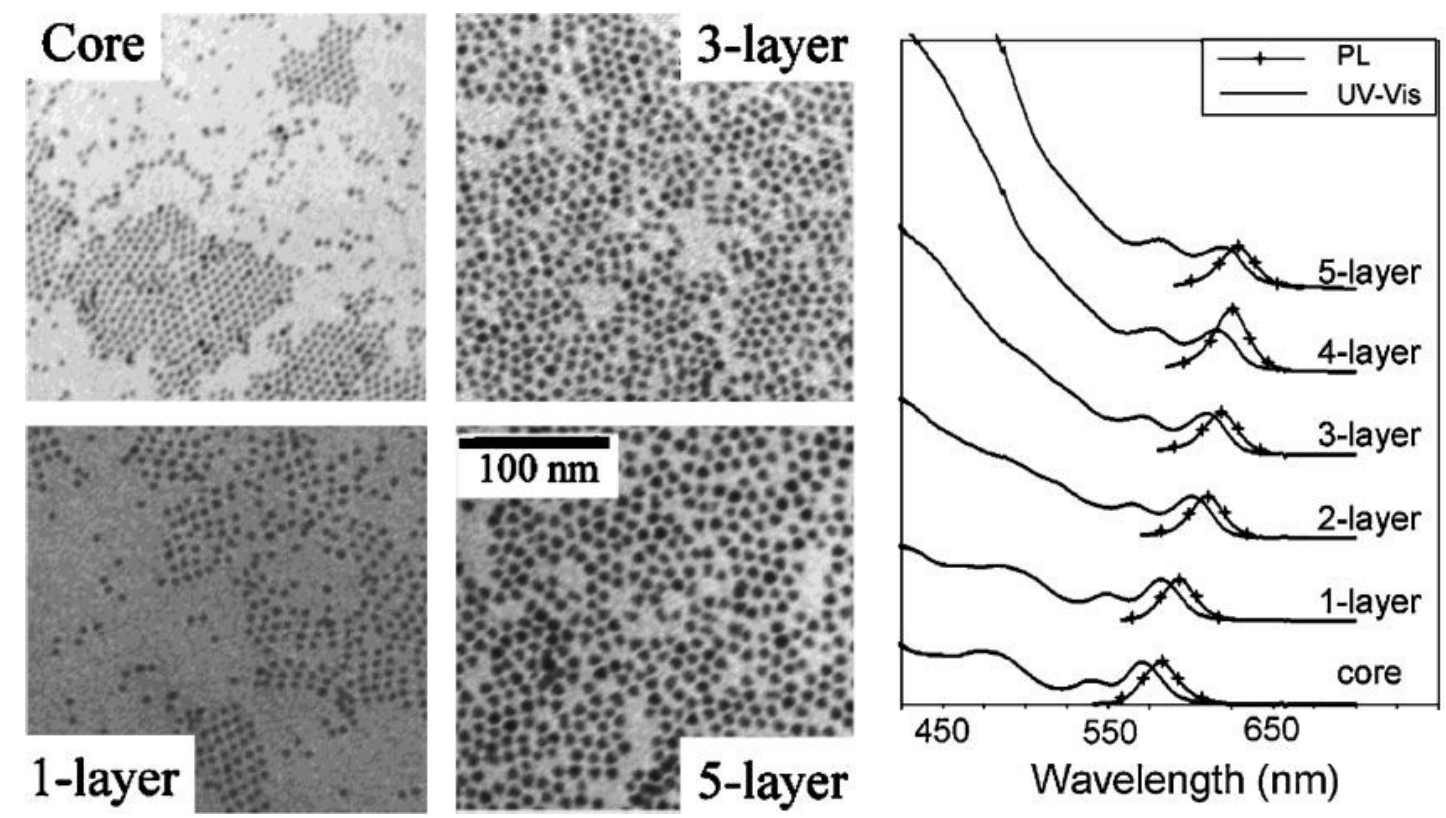

Fig.1.8 Left panel: TEM images of CdSe NCs depicting the increase in diameter upon growth of several monolayers of a CdS shell by means of the SILAR technique. Right panel: UV/Vis and PL spectra of samples with different shell thicknesses. ${ }^{[18]}$

\subsubsection{Multiple Shell QDs}

One of the motivations for the synthesis of QDs containing a multiple-layer shell was the difficulty to respond simultaneously to the requirements of appropriate electronic (bandgap, band alignment) and structural (lattice mismatch) parameters for most binary CS QDs. In particular the lattice mismatch between the core and shell materials strongly limits the possibility to grow a shell with significant thickness without deteriorating the photoluminescence properties. The energy band alignments of two possible types of core/shell/shell (CSS) QDs are depicted in Figure 1.9. Such multiple shell structures provide the combination of low strain, provided by the intermediate layer serving as a "lattice adapter" and efficient passivation and 
charge-carrier confinement assured by the outer shell. Furthermore, the CSS system offers higher oxidation stability than the CS system. CSS QDs with a CdSe core which have been investigated include an intermediate layer of CdS and an outer shell of $\mathrm{ZnS} .{ }^{[19]}$ Uniform shell coverage was observed only for graded shells (e.g., CdS/ZnS) and was found to be critical to achieving QYs close to unity. In this context, a multishell structure consisting of $\mathrm{CdSe}$ core $\mathrm{CdS} / \mathrm{Zn}_{0.5} \mathrm{Cd}_{0.5} \mathrm{~S} / \mathrm{ZnS}$ has been synthesized using the SILAR technique. ${ }^{[20]}$ The monodisperse samples obtained in this study exhibit QYs of 70-85\% and enhanced stability as compared to their binary CS counterparts.

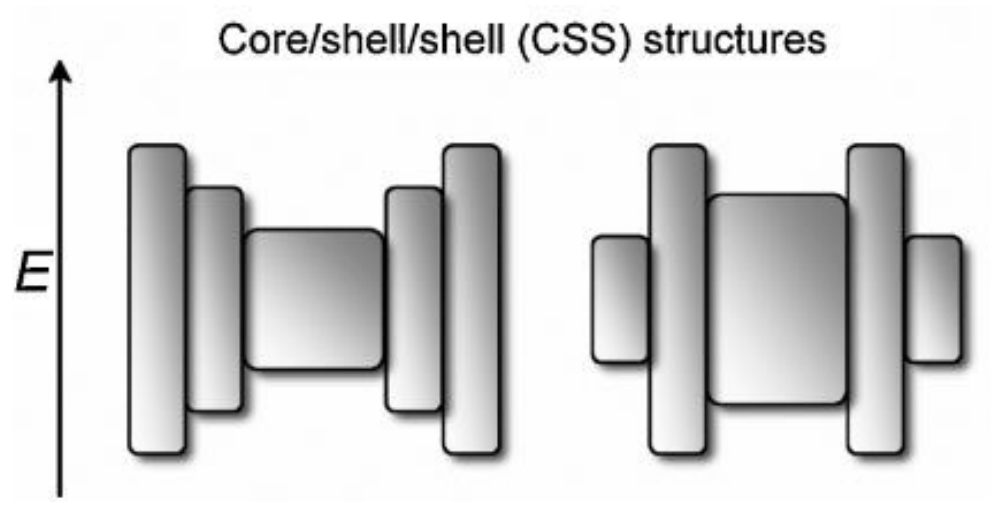

Fig. 1.9 Schematic representation of the energy-level alignment in different CSS structures. The height of the rectangles represents the bandgap energy and their upper and lower edges correspond to the positions of the conduction- and valence-band edge, respectively, of the core (center) and shell materials. ${ }^{[11]}$

\subsubsection{QD/QWNC Heterostructures}

Quantum-dot quantum-well (QDQW) heterostructures represent the special case of CSS QDs in which a lower bandgap layer is embedded between a higher-bandgap core and a higher-bandgap outer shell, and forms a type-I heterostructure with both semiconductors, acting as a QW. The optical properties of QDQWs can thus be easily tuned by changing the QW composition as well as its layer thickness. The latter can be precisely controlled in a SILAR process. Currently, such systems which have been studied extensively include $\mathrm{CdS} / \mathrm{HgS} / \mathrm{CdS},{ }^{[21,}{ }^{22]} \mathrm{ZnS} / \mathrm{CdS} / \mathrm{ZnS}^{[23]}$ and $\mathrm{CdS} / \mathrm{CdSe} / \mathrm{CdS}$. 
[18] SILAR technique is particularly well adapted for the fabrication of QDQW structures due to the need for precise control of the shell thickness. Figure 1.10 showed a schematic view and energy band diagram of a CdS/HgS QDQW structure with multiple HgS wells.
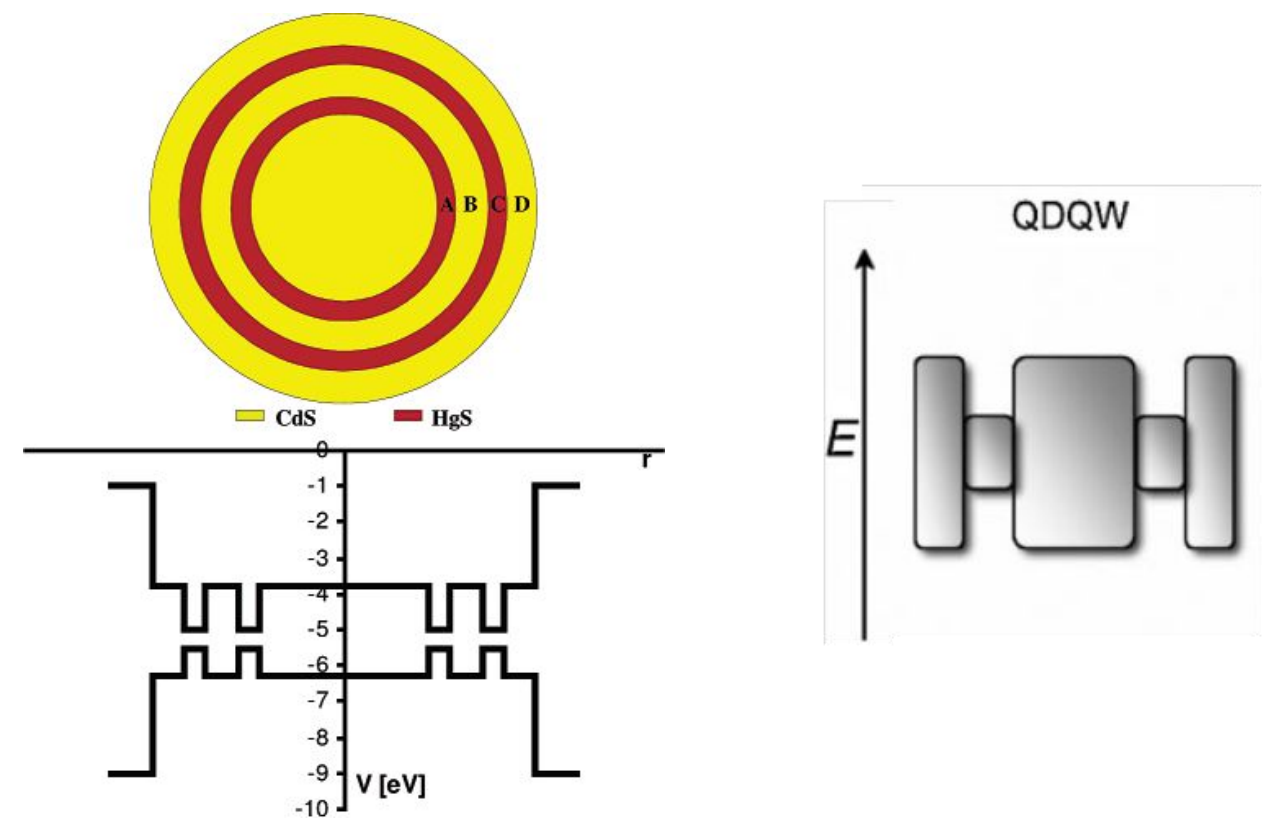

Fig. 1.10 (a) Idealized picture of the double well quantum dot structure with introduction of the $\mathrm{CdS} / \mathrm{HgS}-\mathrm{ABCD}$ nomenclature and the corresponding radial potential for electron and hole. ${ }^{[24]}$ (b) Schematic representation of the energy-level alignment in a QDQW system

A type of "inverse" QDQW NC heterostructure has also been realized with the synthesis of $\mathrm{CdSe} / \mathrm{ZnS} / \mathrm{CdSe} \mathrm{NCs}$, where the larger-bandgap material is embedded between two lower-bandgap ones (Figure 1.9). ${ }^{[25]}$ This heterostructure represents the first structure simultaneously exhibiting two distinct emission wavelengths from the CdSe core and the outer shell (Figure 1.11), a phenomenon that has subsequently been used for the generation of white light. ${ }^{[26]}$ In order to have electronic decoupling of the CdSe core and outer shell, the $\mathrm{ZnS}$ intermediate shell must have a minimum thickness of around three monolayers. ${ }^{[25]}$ Further photophysical studies concerning the energy transfer between the CdSe core and outer shell have recently been carried out on this system. ${ }^{[27]}$ 


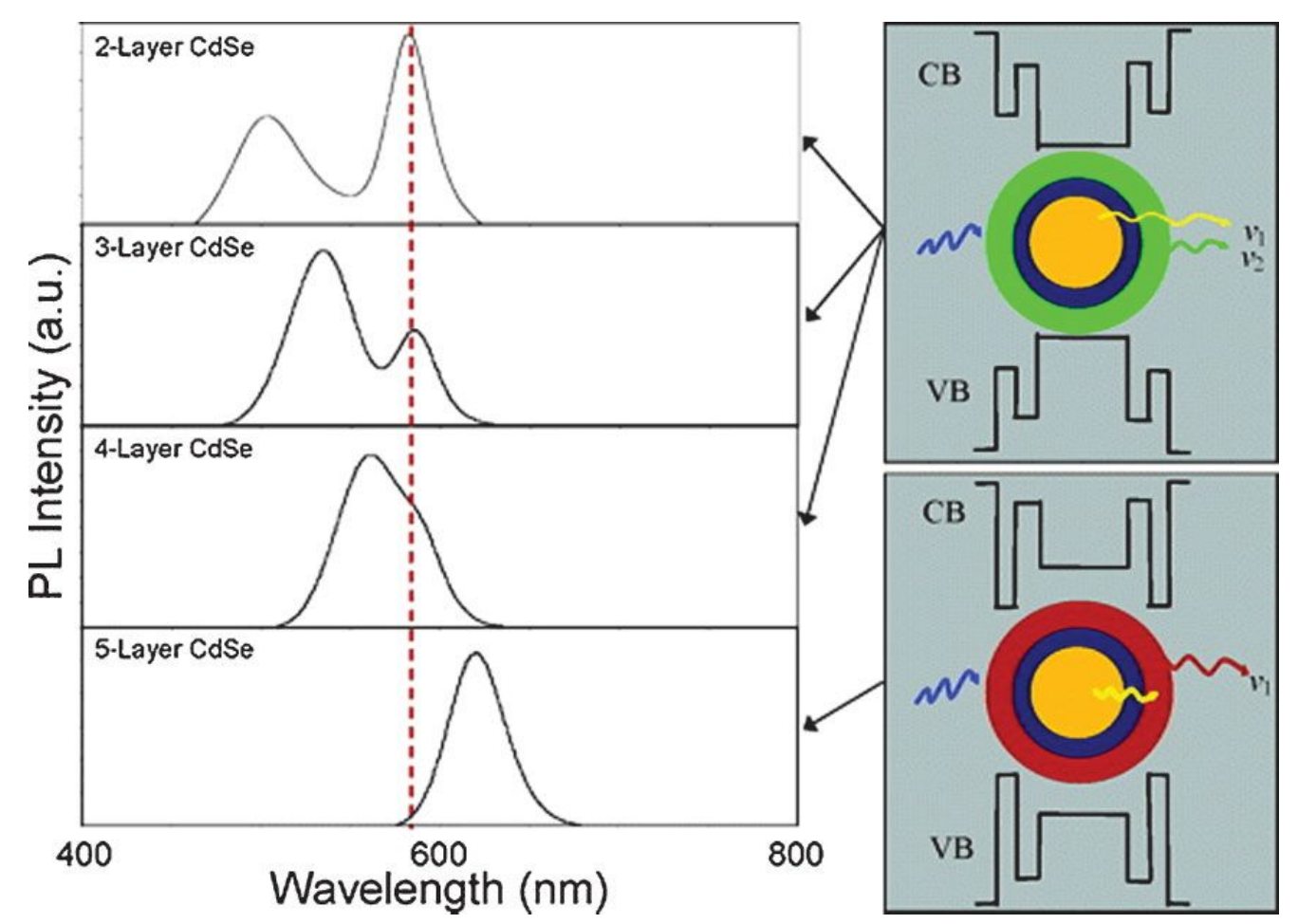

Fig. 1.11 CdSe/ZnS/CdSe CSS NCs. Left: PL spectra as a function of the outer CdSe shell thickness. Right: Corresponding energy-band structures and schemes displaying the different absorption and emission processes. ${ }^{[25]}$

\subsection{Device Application of Colloidal QDs}

Strong bandgap tunability through the quantum size effect, combined with high photoluminescence efficiency and favorable electronic properties, make colloidal QDs attractive for a diverse range of optoelectronic device application. Efforts to harness these properties have led to many successful applications in photovoltaics ${ }^{[28]}$, light-emission $^{[29]}$, photodetection ${ }^{[30]}$ and thermoelectrics. ${ }^{[31]}$ In this section, the progress of QD-based LEDs and solar cells is briefly reviewed.

\subsubsection{QD-based LEDs}

QDs-based light-emitting diodes (QLEDs) have attracted intense research and commercialization efforts over the last decade. ${ }^{[32]}$ They have several advantages compared to organic LEDs (OLEDs), such as highly tunable wavelength, smaller bandwidth of the emission peak, and better stability which results in longer life times. 
Typical QLEDs consist of a QD emitting layer (EML) sandwiched between an organic electron transport layer (ETL) and an organic hole transport layer (HTL). An example is seen in Figure 1.12, which shows the schematic layer structure of a $\mathrm{PbSe}$ QLED and the EL spectra (broad NIR emission) of the QLEDs with different sized PbSe QDs.

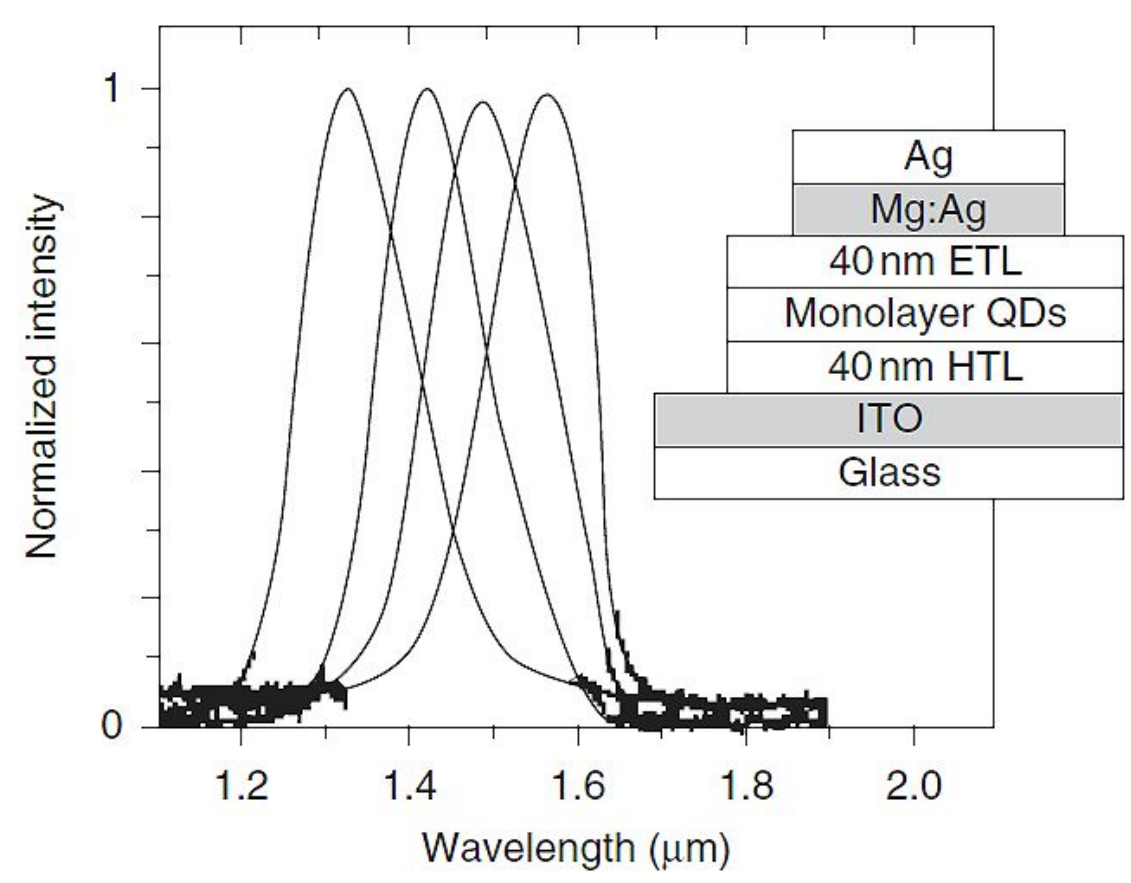

Fig. 1.12 PbSe QDs based QLED device structure and NIR-EL spectra using different sized QDs (ETL: electron transport layer; QDs: quantum dots, HTL: hole transport layer, ITO: indium tin oxide) ${ }^{[33]}$

Since the first QLED was reported by Colvin et al. in 1994[34], visible QLEDs emitting red, green, and blue light have been demonstrated, and their performance has been improved significantly over the last decade. The EQEs of typical QLEDs has evolved from the early demonstration of $0.001 \%$ in $1994^{[34]}$ to $7 \%$, a result reported by QD Vision in 2009 ${ }^{[31]}$ (Figure 1.13). Using core-shell QDs as the emitters allowed one to significantly increase the efficiency of radiative recombination and device internal quantum efficiency. ${ }^{[35]}$ To achieve higher injection currents, heavily doped electron transporting layers were introduced, whereas the thickness of the emitting layer was reduced down to one or two monolayers of highly luminescent semiconductor QDs. ${ }^{[36]}$ One important breakthrough was achieved in 2002 by Bawendi's group, who demonstrated efficient red QD-LEDs with an EML comprising 
a monolayer of $\mathrm{CdSe} / \mathrm{ZnS}$ QDs. The devices take advantage of efficient excitonic energy transfer from fluorescent organic materials, whereas the problem of slow charge transport within QDs is avoided. ${ }^{[36]}$

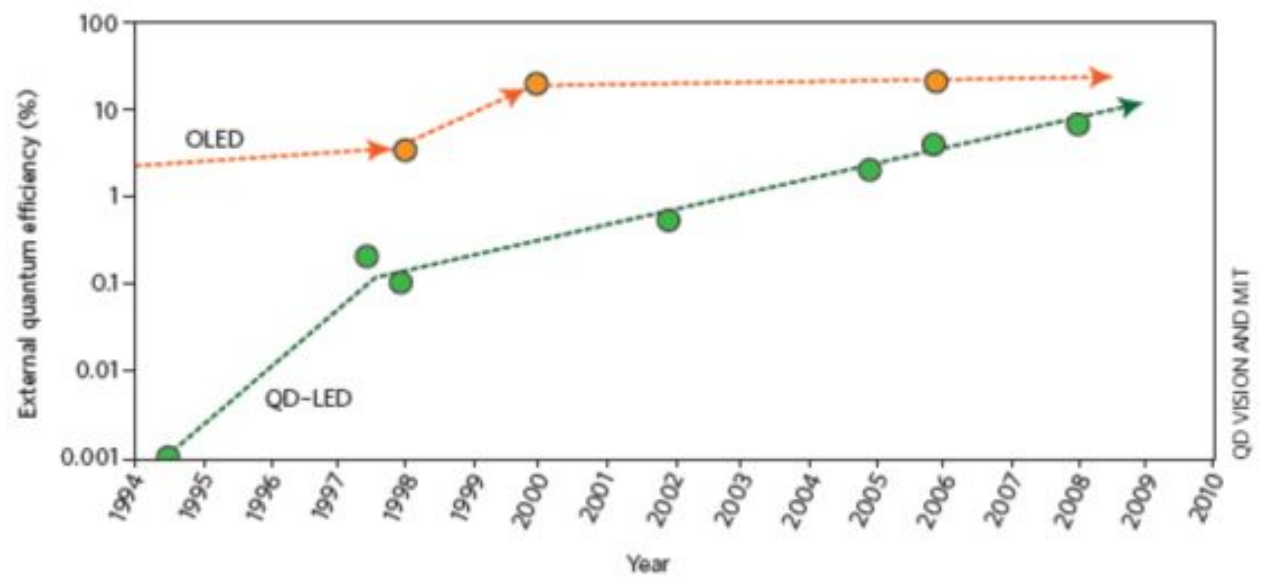

Fig. 1.13 Development of QLEDs (green circles) and OLEDs (orange circles) over the time ${ }^{[34]}$

Since then, QD-LEDs incorporating a monolayer of QDs between an organic electron transport layer (ETL) and an HTL, as shown schematically in Figure1.14, have continuously held the record for QD-LED efficiency until recently. Numerous studies have been devoted to improving their performance and understanding the mechanism behind their operation. ${ }^{[37-39]}$
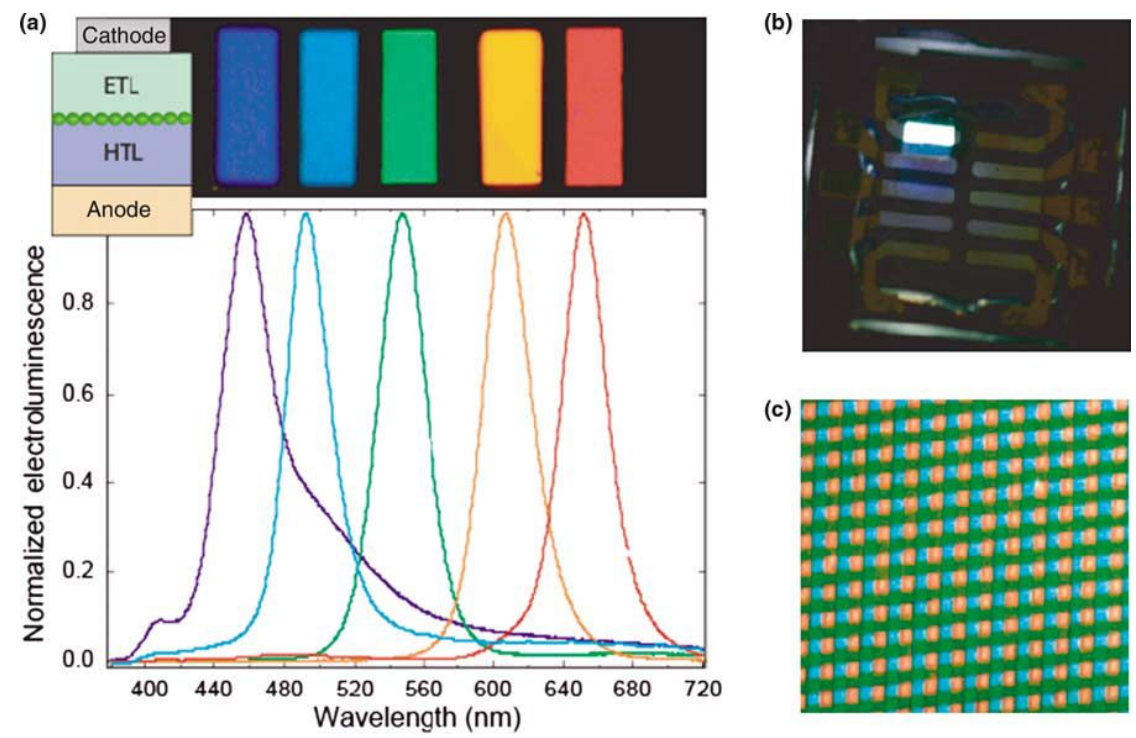

(c)

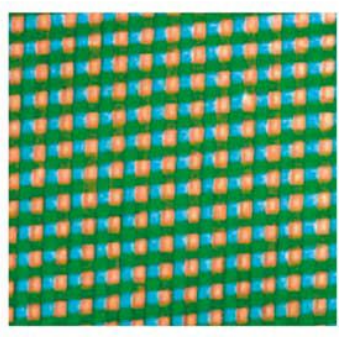

Fig. 1.14 (a) Photographs and spectra of multicolor QD-LEDs that sandwich the QD monolayer using the same organic charge transport layers on either side of the having the cross-sectional geometry 
shown in the inset. Micro contact printing of QDs enables mixing of red, green, and blue QDs within a single monolayer to create a single white light-emitting active layer (b) or side-by-side deposition of $25 \times 25 \mu \mathrm{m}$ red, green, and blue pixels (c). (Figure courtesy of Polina Anikeeva. $)^{[40]}$

As shown in Figure 1.14 a, careful material selection enabled EL from blue, cyan, green, orange, and red QD monolayers sandwiched between the same two organic layers [hole transporting spiro-N,N'-diphenyl-N,N-bis(3-methylphenyl)-(1,1'-biphenyl)-4,4'-diamine (spiroTPD) and electron-transporting 2,2',2”-(1,3,5-benzenetriyl)-tris(L-phenyll-H-benzimidazole) (TPBi)]. ${ }^{[37]}$ These high efficiency, multicolor QD-LEDs were made possible by the development of QD contact printing, in which single monolayers of QDs can be transferred, solvent free, from a parylene-C coated poly(dimethylsiloxane) (PDMS) stamp onto an organic charge transportlayer. ${ }^{[38]}$ Figure 1.14 c shows a display with side-by-side $25 \times 25 \mathrm{~mm}$ RGB pixels, which was created in only two contact printing steps. First, lines of red QDs at $25 \mu \mathrm{m}$ pitch were printed, followed by orthogonal lines of green QDs. The blue emission comes from the HTL, TPD. Unlike most white light sources that require stacking of different color pixels or down conversion schemes, Anikeeva et al. ${ }^{[39]}$ demonstrate that it is possible to fabricate a white LED by incorporating a monolayer of mixed RGB QDs between HTL and ETL. Figure $1.14 \mathrm{~b}$ depicts this white QD-LED, which has an EQE of $0.36 \%$ at $10 \mathrm{~V}$ applied bias and a CRI of 87.
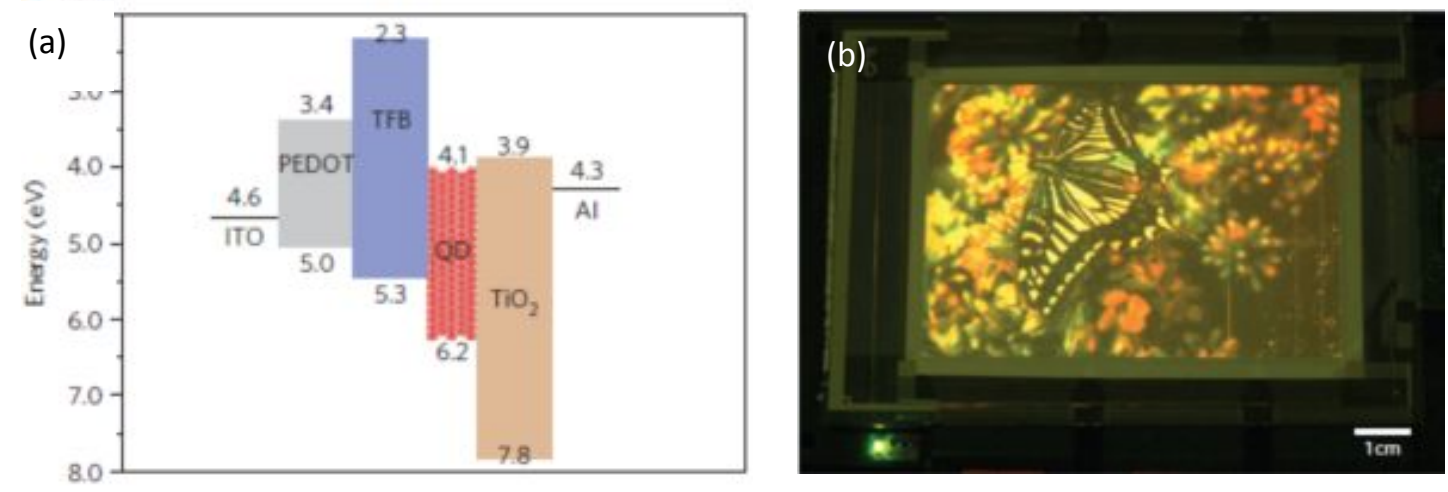

Fig. 1.15 Energy diagram of QD-LEDs and full-color QD display (a) Energy band diagram of QD-LEDs using $\mathrm{TiO}_{2}$ and polymer TFB as the ETL and HTL, as well as quantitative energy levels. (b) Electroluminescence image of a 4-inch full-color QD display using a HIZO TFT backplane with a 
$320 \times 240$ pixel array. ${ }^{[41]}$

Most recently, a team from the Frontier Research Laboratory at the Samsung Advanced Institute of Technology succeeded in developing the world's first full-color quantum dot display. ${ }^{[41]}$ Layers of blue-emitting $\mathrm{CdS} / \mathrm{ZnS}$ core/shell QDs, green-emitting $\mathrm{CdSeS} / \mathrm{ZnS}$ core/shell QDs, or red-emitting CdSe/CdS/ZnS core/shell/shell QDs were integrated into a ITO/PEDOT:PSS/polymer $\mathrm{TFB} / \mathrm{QDs} / \mathrm{TiO}_{2} / \mathrm{Al}$ device structure as the emitters (Figure 1.15). The wavelengths at the EL peak for the red, green and blue QD-LED were 615, 530, and $480 \mathrm{~nm}$, respectively. The respective maximum brightness values were 16,380, 6,425 and 423 $\mathrm{cd} / \mathrm{m}^{2}$. The maximum power efficiency of the red QD-LED was $4.25 \mathrm{~lm} / \mathrm{W}$. They have developed an elegant solvent-free transfer printing method to deposit the QDs multilayer onto the TFB HTL. The adoption of this method enabled large-scale quantum dot displays to be produced and moreover, the luminous efficiency was improved as QDs can be rearranged tightly in a process of transferring quantum dots to the substrates. Similar displays were made not only on glass but also on plastic substrates, predicting well for producing flexible QD displays in the future.

QD Vision Inc. recently reported a color-saturated, red QD-LED using an unique inverted organic-inorganic hybrid device structure and colloidal CdSe-CdS (CS) QD emitters. The strong electronic coupling of QDs to an adjacent layer of $\mathrm{ZnO}$ nanocrystals (which form the electron transport layer) facilitates charge transfer, which is responsible for both injecting electrons and maintaining an optimal charge balance. Devices were reported with a luminous efficiency of $19 \mathrm{~cd} / \mathrm{A}$, corresponding to an external quantum efficiency of $18 \%$ (which is close to the theoretical maximum of $20 \%$ ) and an internal quantum efficiency of $90 \%{ }^{[42]}$

Although the underlying device physics of QD-LEDs have been sufficiently addressed, the EQE of typical QD-LEDs is still relatively low, especially in the short-wavelength regime. To further improve the performance of QD-LEDs emitting green and blue light, the following improvements are needed: better energy alignment between QDs and HTL/ETL, higher stability of HTL/ETL, better surface design of QDs, and more efficient excitonic energy transfer and more facile carrier injection from HTL/ETL into the QDs. 


\subsubsection{QD-based Solar Cells}

The growing energy market is calling demand for clean and renewable energy these days, such as solar energy. Semiconductor QDs are considered as light-absorbing materials well suited for photovoltaic applications provided their potential to achieve high efficiency and the benefits of low-cost materials and processing. QD-based solar cells have undergone considerable progress over the recent years and may make a significant technological impact in the near future.

Several types of QD-based solar cell structure which enable efficient light absorption and separation of positive and negative charge carriers have been explored, including QD sensitized solar cells ${ }^{[43]}$, depleted heterojunction QD solar cells ${ }^{[44]}$, Schottky QD solar cells ${ }^{[45]}$, and quantum junction solar cells ${ }^{[46]}$. In this section, we mainly review the recent work on solar cells based on QD solids, which have yielded the best conversion efficiency so far.

In a report published in 2008, Luther et al. used the QD/metal Schottky structure to correlate the size-tuned band positions of the QD film with the known work functions of various metals (Figure $1.16 \mathrm{a}, \mathrm{b}$ ) and further quantified the equilibrium free carrier density within the QD film through Mott-Schottky analysis (Figure $1.16 \mathrm{c}){ }^{[47]}$ Remarkably, the simple Schottky structure can be optimized to achieve a solar conversion efficiency> 4.5\% through tuning the QDs size. ${ }^{[4]}$ Unfortunately, Schottky barriers impose a low upper bound on the built-in voltage and thus the $\mathrm{V}_{\mathrm{OC}}{ }^{[49]}$ For this reason, all reported Schottky devices showed small open-circuit voltages. ${ }^{[50]}$ Figure $1.16 \mathrm{~d}$ shows the $\mathrm{J}-\mathrm{V}$ characteristics for a high-performance Schottky QD solar cell, with a $\mathrm{V}_{\mathrm{OC}}$ of $0.47 \mathrm{~V}$, which is low considering that the active QDs layer have a banggap of $1.6 \mathrm{eV} .^{[51]}$ 

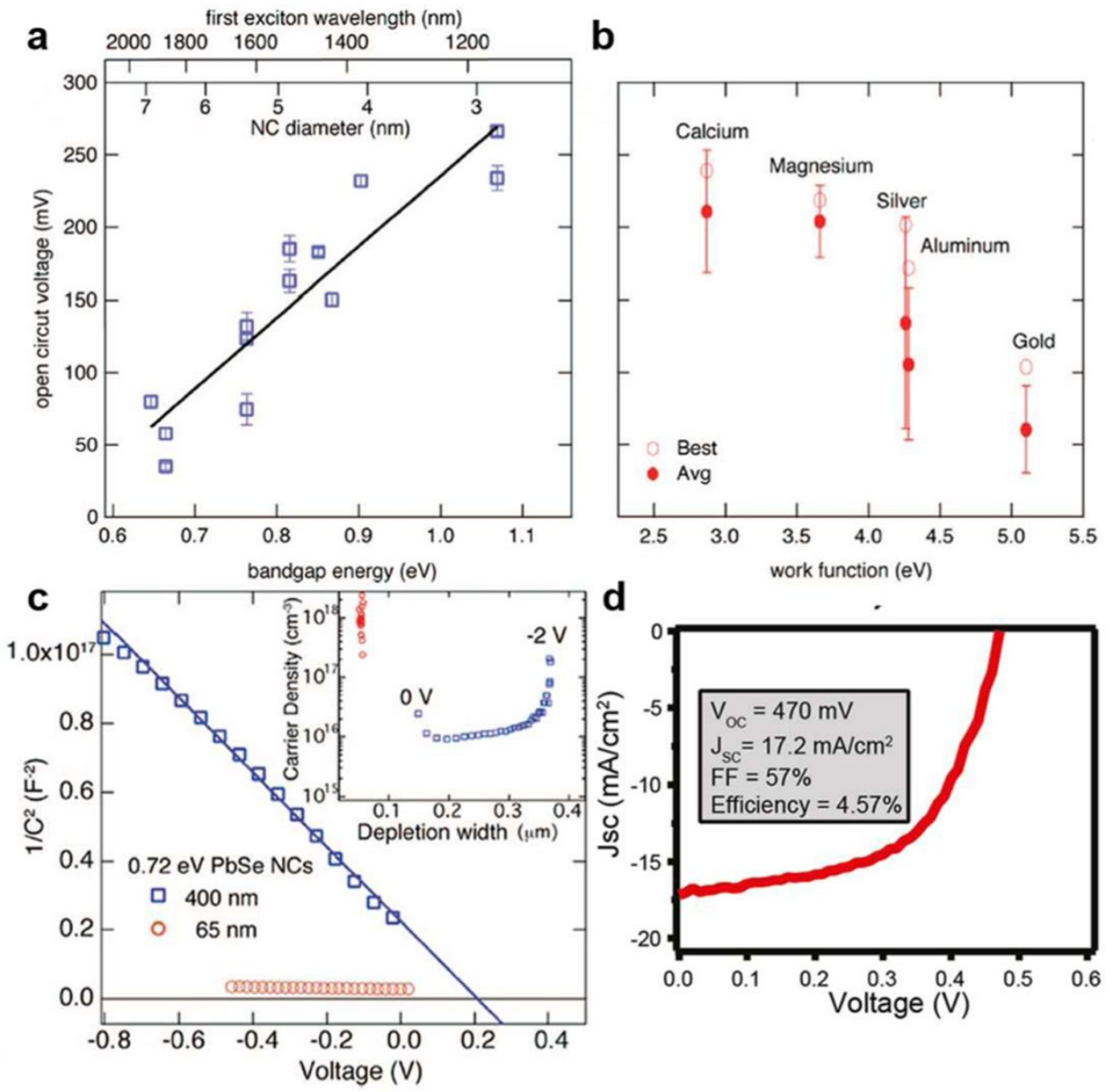

Fig. 1.16 VOC as a function of (a) QD size (and thus bandgap energy and first excitonic wavelength) and (b) Schottky contact work function. (c) Mott-Schottky analysis of PbSe CQD films of 65 and 400 $\mathrm{nm}$ thicknesses. Reprinted with permission from ref 47.Copyright 2008 American Chemical Society. (d) $\mathrm{J}-\mathrm{V}$ curves of champion Schottky QD solar cell exhibiting $\mathrm{V}_{\mathrm{OC}}$ of $0.47 \mathrm{~V}$ and $\eta$ of $4.57 \%$. Reprinted with permission from ref 47.Copyright 2011 American Chemical Society. ${ }^{[1]}$

Sargent's groups recently reported the fabrication and characterization of depleted heterojunction QD solar cells using PbS QD thin films. ${ }^{[52]}$ A schematic illustrating the layer structure of a standard device is shown in Figure 1.17 a. Two classes of fluorine-doped tin oxide (FTO) substrates were used in this case, one having an appreciably deeper work function $(\Phi)$ than the other. $\mathrm{TiO}_{2}$ films with different thicknesses were deposited by atomic layer deposition (ALD) on top of the two classes of FTO. A PbS QD film was then deposited via a layer-by-layer spin-casting process using 3-mercaptopropionic acid (MPA) for the solid-state exchange. The quantum dots benefited from a hybrid passivation technique, which 
included exposure to halides in the final stages of their synthesis. ${ }^{[52]}$ The standard thickness of the QD films was $380 \mathrm{~nm}$ and a $\mathrm{MoO}_{3} / \mathrm{Au} / \mathrm{Ag}$ stack was evaporated as the top electrode. The cross-sectional SEM images of the completed devices (with 10 $\mathrm{nm} \mathrm{TiO}_{2}$ films) are shown in Figure $1.17 \mathrm{~b}$ (shallow- $\Phi$ FTO) and Figure $1.17 \mathrm{c}$ (deep- $\Phi$ FTO).The shallow- $\Phi$ FTO device has achieved a conversion efficiency of over $8.5 \%$ under AM1.5 solar illumination, which is the best reported performance of QD solar cells so far.
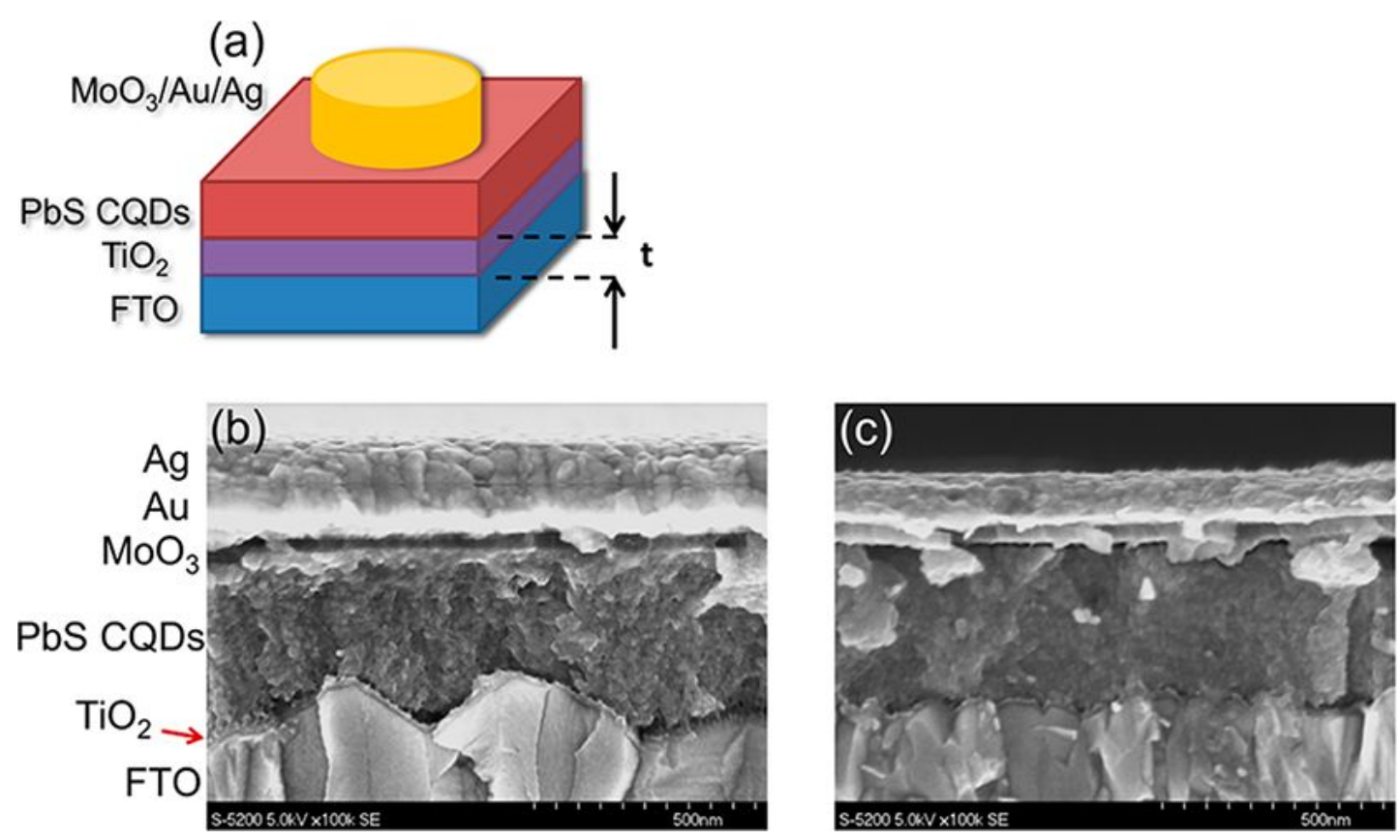

Fig 1.17 QD solar celldevice architecture. (a) Schematic of a standard QD device. The thickness of the $\mathrm{TiO}_{2}$ layer $(\mathrm{t})$ was varied in the devices. The films shown are prepared on a glass substrate. (b) Cross-sectional SEM image of a QD device on shallow-work function FTO with a $10 \mathrm{~nm}$ ALD $\mathrm{TiO}_{2}$ film. (c) Cross-sectional SEM image of a QD device on deep work function FTO with a $10 \mathrm{~nm} \mathrm{TiO}_{2}$ film.

\subsubsection{Challenges Facing QD-based Devices}

Despite the recent rapid progress, the performance of QD-based devices is still generally poor. Both the EQEs of QD-LEDs and the power conversion efficiencies of 
QD-based solar cells reported in the literature are limited to a few percent. Common efficiency-limiting factors in current QD-based devices include charge trapping and recombination at QD surfaces, poor charge injection into QDs and slow transport in QD solids, and unbalanced charge injection in QD device structures. Performance enhancement of these devices requires extensive further work in two aspects: (i) improvement of the optical and electronic properties of colloidal QDs by optimizing their synthesis recipes, manipulating their surface chemistry, and tailoring their heterostructures; (ii) exploration of new device architectures, alternative charge transport materials, and more effective processing of QD thin films.

\subsection{Research Focus}

This thesis aims to achieve desirable optical properties of colloidal CdSe NC QDs by strain and energy band engineering in core/multishell NC heterostructures and explore their optoelectronic applications by demonstration of working LEDs based on these NC heterostructures. The research focuses on two types of CdSe NC heterostructures, as briefly described below.

Chapter 2 presents the synthesis and characterization of CdSe QDs with a strain compensated bilayer shell. The core/multishell QDs enjoy the benefits of excellent exciton confinement by a $\mathrm{ZnS}$ intermediate shell and strain compensation by a $\mathrm{ZnCdS}$ outer shell, leading to high luminescence efficiencies and great color stability.

Chapter 3 describes the synthesis and characterization of novel CdS/CdSe/ZnS QDQW nanoparticles. By tailoring the CdSe QW width as well as the core/shell sizes, high-efficiency blue light emission at $\sim 470 \mathrm{~nm}$ is achieved. The QDQWs are incorporated into LED structures with different organic hole transport layers, and blue EL from QDQWs was successfully obtained. 


\section{Chapter 2 Synthesis of CdSe QDs with strain compensated multishell for high-efficiency light emission}

The exploration for QD device applications have been made based on the recent rapid progress in the synthesis of high-quality colloidal QDs. As discussed in section 1.2, low-cost and controllable synthetic protocols have been developed for synthesis of core QDs as well as core/shell structures. Colloidal II-VI semiconductor QDs has attracted scientists' interest due to their unique optical properties and potential uses for high-efficiency energy conversions. For example, CdSe and CdS QDs are chosen as efficient emitters for developing QD-LEDs with high color purity (narrow excitonic peak) and tunable emission wavelength over the entire visible range.

In this chapter, we synthesized CdSe core QDs using a modified hot-injection method published by Murray ${ }^{[1]}$ and Peng. ${ }^{[53]}$ Two different multishell structures $\left(\mathrm{ZnS} / \mathrm{Cd}_{0.5} \mathrm{Zn}_{0.5} \mathrm{~S}\right.$ and $\left.\mathrm{Cd}_{0.5} \mathrm{Zn}_{0.5} \mathrm{~S} / \mathrm{ZnS}\right)$ were designed and synthesized via the SILAR method. ${ }^{[18]}$ Structural and optical characterization of the two different QDs are conducted. Finally, QD-LEDs with an emitting layer comprising the core/multishell QDs were fabricated and their characteristics were compared.

\subsection{Synthesis of CdSe QDs with strain compensated multishell}

\subsubsection{Synthesis of CdSe core QDs}

TOPO/ODA-capped CdSe core QDs were synthesized using standard hot injection method. For a typical reaction, the mixture of $1 \mathrm{mmol} \mathrm{CdO}$ powder, $1.27 \mathrm{~mL}$ oleic acid (OA) and $12.5 \mathrm{~mL}$ 1-octadecene (ODE) in a three-necked flask was evacuated for $20 \mathrm{~min}$ at room temperature and $10 \mathrm{~min}$ at $100^{\circ} \mathrm{C}$, and then heated to about $250{ }^{\circ} \mathrm{C}$ under $\mathrm{N}_{2}$ atmosphere flow to obtain an optically clear solution. After that, the solution was allowed to cool to room temperature, and octadecylamine (ODA) 
(7.5 g) and trioctylphosphine oxide (TOPO) (2.5 g) were added and the solution was reheated to $250^{\circ} \mathrm{C}$. At this temperature, a selenium solution containing $0.6 \mathrm{~g}$ of $\mathrm{Se}$ dissolved in a mixture of $8 \mathrm{~mL}$ of TOP and $2 \mathrm{~mL}$ of ODE was quickly injected into the reaction flask. The following equation (1) shows the reaction mechanism during the injection.

After the injection, the temperature was maintained at $250^{\circ} \mathrm{C}$ for about $10 \mathrm{~s}$ for the growth of CdSe QDs. After the growth step, the heating mantle was removed to cool the solution to room temperature. An extraction procedure was then used to purify the QDs by removing the side products and unreacted precursors. ${ }^{[53]}$ Figure 2.1 illustrates the process of hot-injection method.

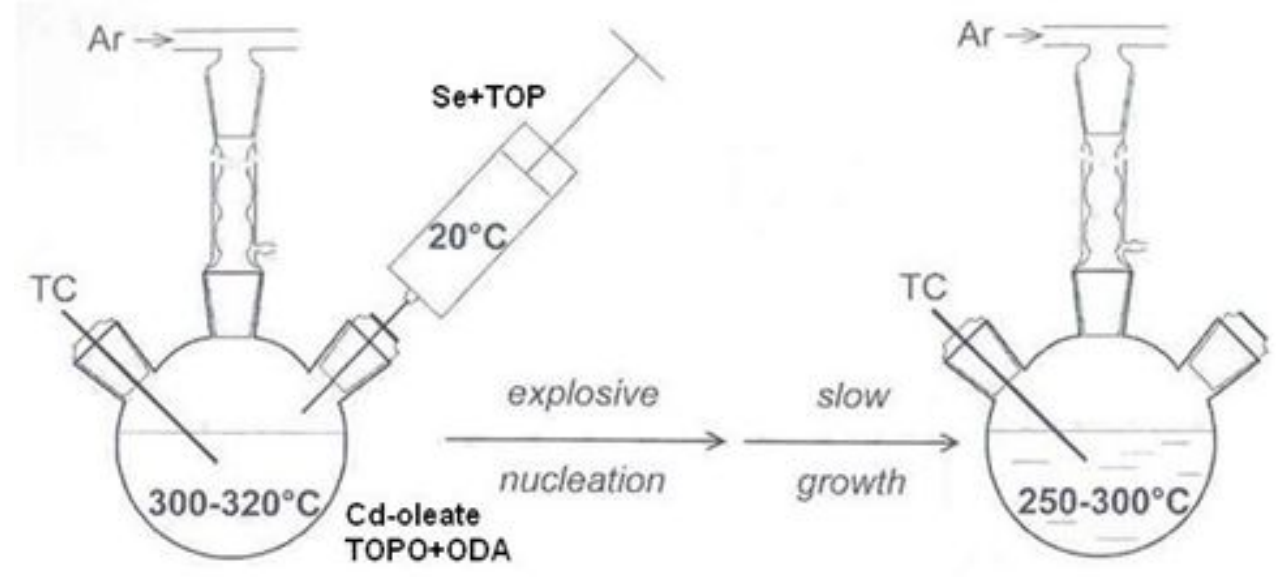

Fig. 2.1 General hot-injection method for synthesis of CdSe core QDs. This picture was taken from Y. Q. Zhang's Dissertation at West Virginia University, 2012.

The absorption and photoluminescence (PL) spectra of CdSe core QDs dissolved in hexane were measured, using a Hitachi U-3900H UV-Vis spectrophotometer and a Hitachi F-7000 fluorescence spectrophotometer, respectively. Figure 2.2 displays the temporal evolution of the absorption and PL spectra of CdSe core QDs grown at 250 ${ }^{\circ} \mathrm{C}$ for $10 \mathrm{~s}$. The first excitonic peak of the obtained CdSe QDs was about $528 \mathrm{~nm}$, whereas the corresponding PL peak showed a red shift known as Stoke shift. The observed $13 \mathrm{~nm}$ Stokes shift is consistent with the data reported in the literature. ${ }^{[54]}$ The existence of multiple excitonic absorption peaks and the narrow full width at half maximum (FWHM) of the band edge PL spectra (25 nm of FWHM) of the QDs 
suggests that the resulting QDs dissolved in Hexane are nearly monodispersed and have narrow size distribution. Based on absorption and PL measurements, the PL QY of the CdSe core QDs in hexane was determined to be $\sim 10 \%$, suggesting good passivation of the core QDs by the ODA-TOPO ligands.

The sizes of the QDs can be calculated based on their first excitonic absorption peak positions using the following empirical equation, derived by Peng and co-workers. ${ }^{[53]}$

$\mathrm{D}=\left(1.6122 \times 10^{-9}\right) \lambda^{4}-\left(2.6575 \times 10^{-6}\right) \lambda^{3}+\left(1.6242 \times 10^{-3}\right) \lambda^{2}-(0.4277) \lambda+(41.57)$

In the above equation, $\mathrm{D}(\mathrm{nm})$ is the diameter of a given QD sample, and $\lambda(\mathrm{nm})$ is the wavelength of the first excitonic absorption peak of the sample. The evolution of the particle size during growth of the CdSe QDs at $250{ }^{\circ} \mathrm{C}$ was calculated to be approximately $2.64 \mathrm{~nm}$.

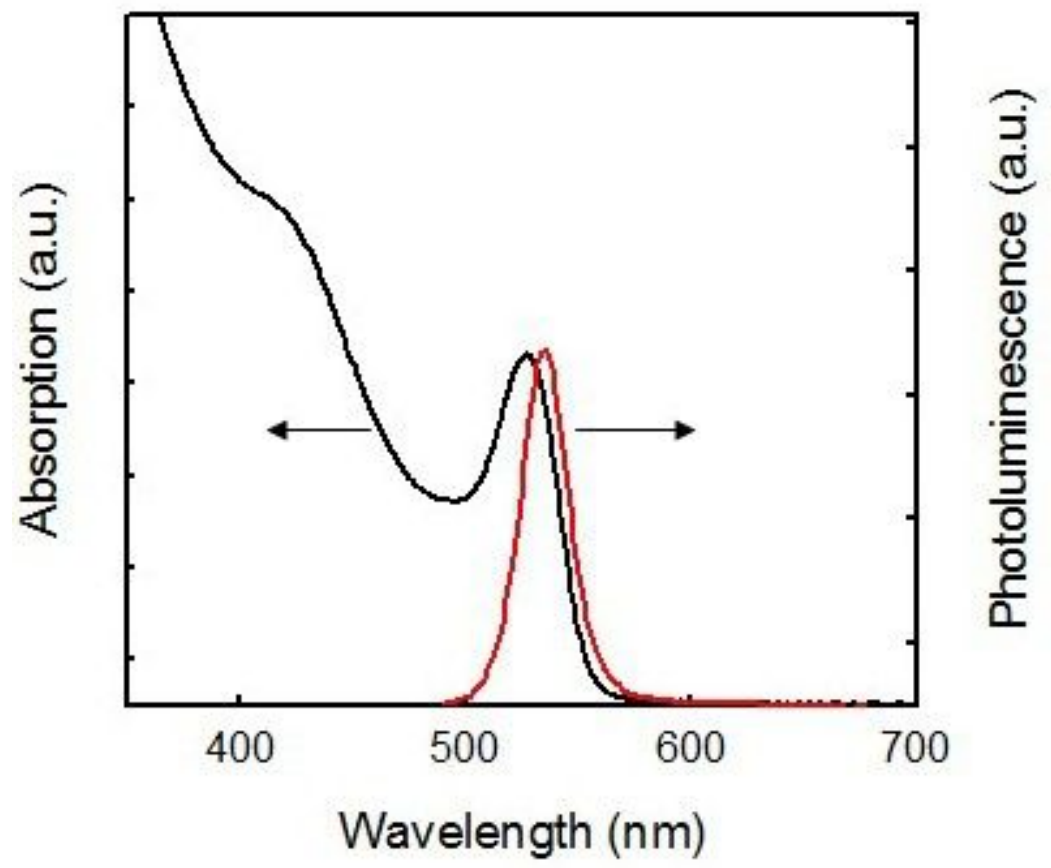

Fig. 2.2 Absorption and PL spectra of CdSe QDs emitting green light (528 nm)

\subsubsection{Growth of core/shell (CS) QDs}

Growth of shell outside the core QDs will lead to better passivation, and therefore, result in enhanced luminescence QYs, and greater stability against the harsh processing conditions. ${ }^{[9]}$ This task aims to grow and compare two different structures 
of high-quality core/shell QDs by the SILAR method. ${ }^{[18]}$ To obtain the best structures, 1 6 monolayers of $\mathrm{ZnS}$ and $\mathrm{Cd}_{0.5} \mathrm{Zn}_{0.5} \mathrm{~S}$ shell materials were first grown on the CdSe core and then measured in Hexane. During the synthesis process, both the $\mathrm{ZnS}$ shell and the $\mathrm{Cd}_{0.5} \mathrm{Zn}_{0.5} \mathrm{~S}$ alloying shell are grown one monolayer at a time by alternatively injecting air-stable and inexpensive cationic ( $\mathrm{Zn-}$ or $\left.\mathrm{Cd}_{0.5} \mathrm{Zn}_{0.5^{-}}\right)$and anionic(S-) precursors into the reaction mixture with CdSe core QDs.

Figure 2.3 shows the cross-section and energy band diagrams of the two kinds of CS QDs. The bandgap of the $\mathrm{ZnS}(3.7 \mathrm{eV})$ and $\mathrm{Cd}_{0.5} \mathrm{Zn}_{0.5} \mathrm{~S}(3.0 \mathrm{eV})$ alloying shell materials (calculated as the average bandgap of $\mathrm{CdS}$ and $\mathrm{ZnS}$ ) are larger than that of the CdSe core $(1.96 \mathrm{eV} 2.64 \mathrm{eV})$. Also, the conduction band of the $\mathrm{ZnS}$ shell is of higher energy than that of the CdSe core, while the valence band of the $\mathrm{ZnS}$ shell is of lower energy than that of the CdSe core. This core/shell structure thus has type-I band alignment and enable effective confinement of electrons and holes in the core material. Consequently, a significant improvement in QDs emission QYs can be achieved. ${ }^{[11]}$ In comparison, although the conduction band of the $\mathrm{Cd}_{0.5} \mathrm{Zn}_{0.5} \mathrm{~S}$ shell is of higher energy than that of the CdSe core, the valence band is of lower energy than that of the CdSe core, and therefore forms a type-II band alignment. ${ }^{[1]}$ The staggered band alignment leads to a smaller effective bandgap overlap and a significant red-shift of the emission wavelength.
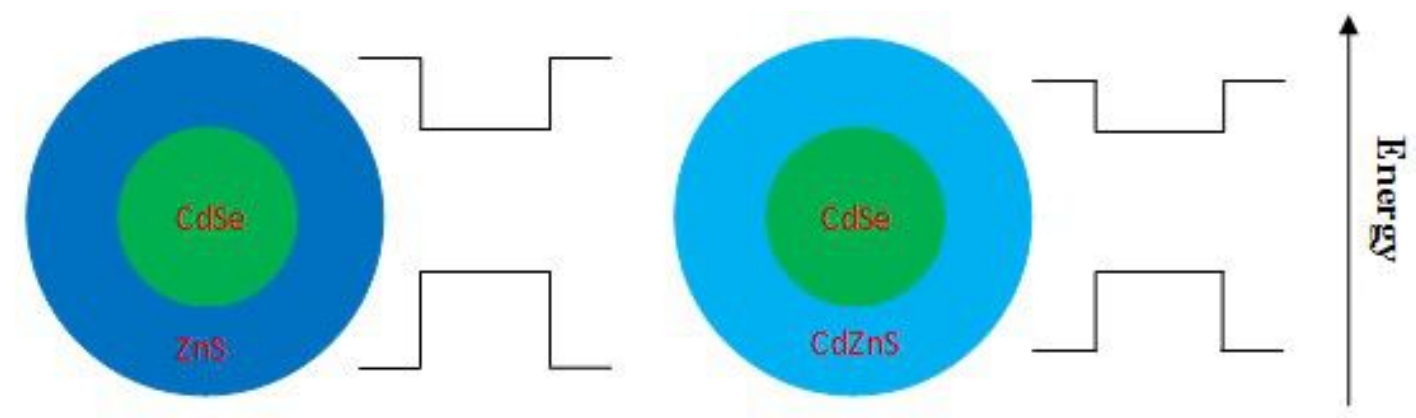

Fig. 2.3 Schematic cross-sections and energy band diagrams of two different CS QDs

In a typical synthesis of the above two types of CS QDs, CdSe core QDs (2.64 $\mathrm{nm}$ in diameter, ) were prepared using the recipe described earlier and mixed with 18 $\mathrm{mL}$ of ODE and $6 \mathrm{~g}$ of ODA in a three-necked flask. The mixture was evacuated similar as the previous core synthesis and then heated to $240{ }^{\circ} \mathrm{C}$ in $\mathrm{N}_{2}$ atmosphere, and $\mathrm{Zn} / \mathrm{Cd}_{0.5} \mathrm{Zn}_{0.5}$ precursor and $\mathrm{S}$ precursor were injected alternatively into the flask for the shell layer growth in the order of $\mathrm{Zn} / \mathrm{Cd}_{0.5} \mathrm{Zn}_{0.5}-\mathrm{S}$ with an interval of $10 \mathrm{~min}$ 
between two successive injections (see details in Appendix 1). The reaction mixture was allowed to cool to room temperature, and followed by QD extraction procedure. Further purification of CS QDs was done by precipitating them with acetone or methanol. ${ }^{[53]}$ Highly pure QDs were obtained for device fabrication by repeating above purification procedure for 2-3 times and followed by filtering. During the shell growth, aliquots of the solution were removed after each monolayer of shell growth with a syringe and tested for optical spectra.
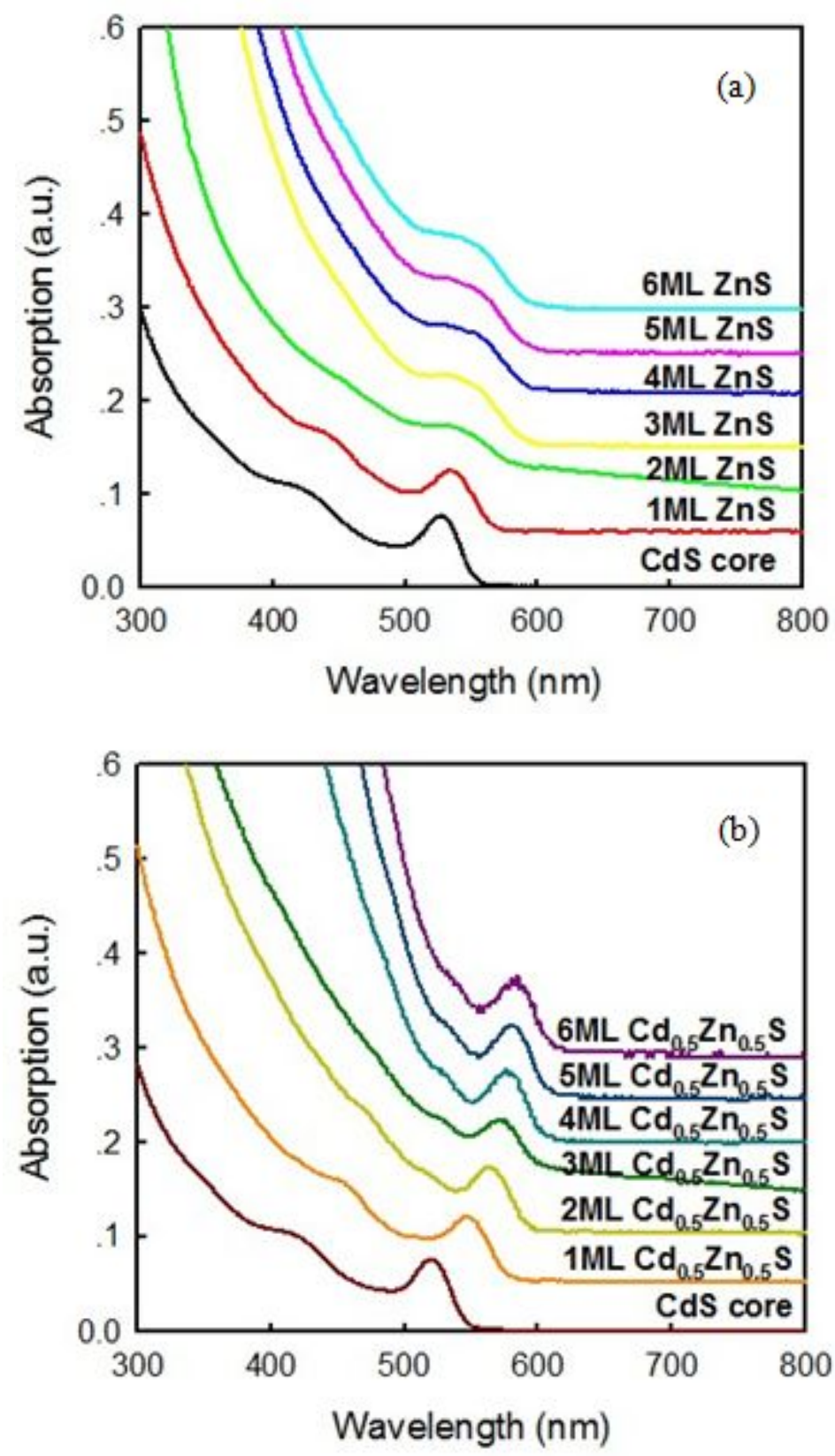

Fig. 2.4 Absorption spectra of CdSe core and (a) CdSe/ZnS CS QDs (b) $\mathrm{Cd}_{0.5} \mathrm{Zn}_{0.5} \mathrm{~S}$ CS QDs with different numbers of monolayers (MLs) 
Figure 2.4 displays the evolution of the absorption spectra upon consecutive growth of the passivating shell. The first excitonic absorption peak of $\mathrm{CdSe} / \mathrm{ZnS}$ core/shell QDs gradually shifts to the longer wavelength from $528 \mathrm{~nm}$ to $550 \mathrm{~nm}$, whereas the $\mathrm{CdSe} / \mathrm{Cd}_{0.5} \mathrm{Zn}_{0.5} \mathrm{~S}$ core/shell QDs exhibits more red shift from $528 \mathrm{~nm}$ to $583 \mathrm{~nm}$. This phenomenon can be explained based on the band structure of both the CS QDs: the wave function of light electrons tunnels into the $\mathrm{ZnS} / \mathrm{Cd}_{0.5} \mathrm{Zn}_{0.5} \mathrm{~S}$ shell, while the wave function of heavy holes has a negligible probability of spreading into the shell. The increased delocalization of the electrons, in the core/shell structure, lowers the confinement energy and therefore the energy of the excited state, leading to a red shift in the optical spectra. As we discussed above, the staggered band alignment of $\mathrm{CdSe} / \mathrm{Cd}_{0.5} \mathrm{Zn}_{0.5} \mathrm{~S}$ core/shell QDs leads to a smaller effective bandgap overlap and much lower energy of the excited state which results in a more significant red-shift than $\mathrm{CdSe} / \mathrm{ZnS}$ core/shell QDs.

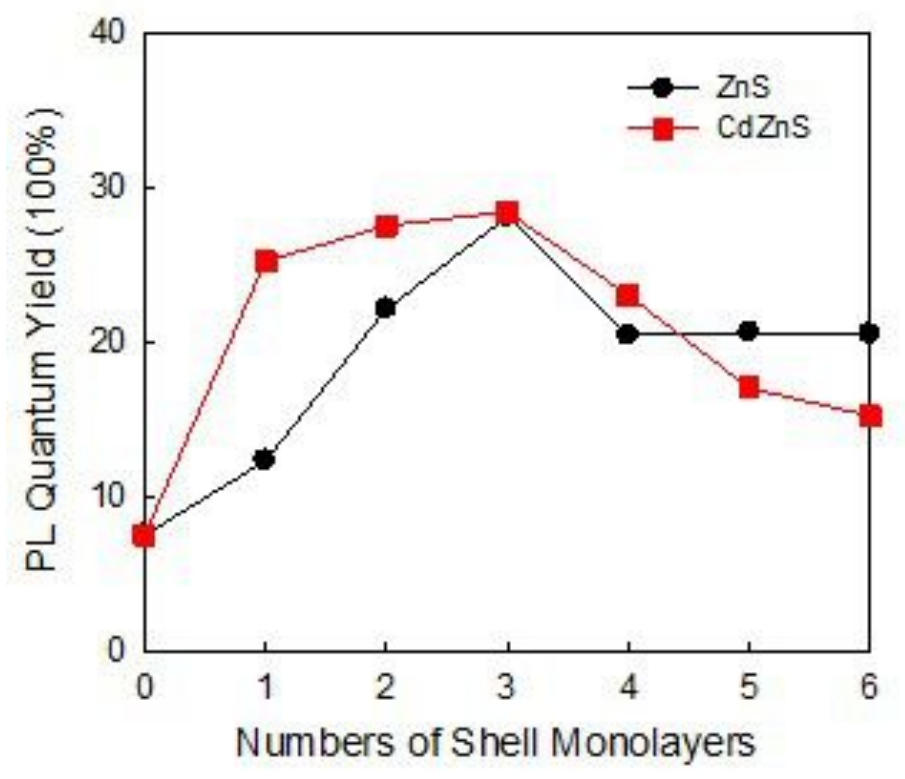

Fig. 2.5 Evolution of the PL quantum yield of CdSe/ $\mathrm{ZnS}$ and $\mathrm{CdSe} / \mathrm{Cd}_{0.5} \mathrm{Zn}_{0.5} \mathrm{~S}$ CS QDs

The evolution of the PL QY of the two core/shell QDs are shown in Figure 2.5. The QY of the CdSe core QDs is on the order of 10\%. The QY of CdSe/ZnS CS QDs is increased from $\sim 10 \%$ to $\sim 30 \%$ after coating with 3 MLs of $\mathrm{ZnS}$ shell, while the QY of $\mathrm{CdSe} / \mathrm{Cd}_{0.5} \mathrm{Zn}_{0.5} \mathrm{~S}$ CS QDs is increased from $\sim 10 \%$ to $\sim 30 \%$. Additional growth of both $\mathrm{ZnS}$ and $\mathrm{Cd}_{0.5} \mathrm{Zn}_{0.5} \mathrm{~S}$ shells leads to an evident decrease in the $\mathrm{QY}$, due to the formation of defects at the core/shell interface. ${ }^{[15]}$ Because of the large lattice mismatch between the core and shell materials (11.6\% for CdSe/ZnS QDs and 7.5\% 
for $\mathrm{CdSe} / \mathrm{Cd}_{0.5} \mathrm{Zn}_{0.5} \mathrm{~S}$ QDs), lattice strain increases as the shell grows thicker. ${ }^{[15]} \mathrm{A}$ partial relaxation of the strain may lead to misfit defect formation, and thus $2 \sim 3$ intermediate shell layers could be chosen for further multishell structure growth.

\subsubsection{Growth of core/shell/shell (CSS) QDs}

Even though $\mathrm{ZnS}$ provides effective electronic passivation of CdSe core QDs, it is not an ideal shell material because its lattice constant is $\sim 12 \%$ smaller than that of CdSe. Tensile strain in the $\mathrm{ZnS}$ shell increases as the shell grows thicker, and eventually relaxes through misfit defect formation, degrading the optical properties of the CS QDs. To mitigate the problem, a thin layer of a semiconductor whose lattice constant falls between those of $\mathrm{CdSe}$ and $\mathrm{ZnS}$, such as $\mathrm{CdS}$, ZnSe, CdS or ZnCdS, is often inserted between the CdSe core and $\mathrm{ZnS}$ shell, forming a core/shell/shell (CSS) structure. ${ }^{[19-20,54-55]}$ The intermediate shell acts as a lattice adapter and a strain-reducing layer, giving rise to a significant improvement of the structural and optical properties of the QDs. In this work, we propose an alternative strategy to reduce strain in CdSe/ZnS QDs and prove its effectiveness in defect reduction and luminescence enhancement. A layer of a material with a lattice constant smaller than that of $\mathrm{ZnS}$, i.e. $\mathrm{ZnCdS}$, is grown outside the $\mathrm{ZnS}$ shell as a strain-compensating layer. The resulting CSS QDs with a strain-compensated shell are expected to have advantages over conventional CSS QDs since the wide bandgap ZnS shell directly covers the core, and provides better passivation and carrier confinement than a strain-reducing intermediate shell in conventional CSS QDs, which typically has a smaller band gap than ZnS. To validate this new strategy, two types of CSS QDs, as shown in Figure 2.6, CdSe/ZnCdS/ZnS QDs and CdSe/ZnS/ZnCdS QDs, denoted as CSS I QDs and CSS II QDs, were synthesized and characterized, respectively. 


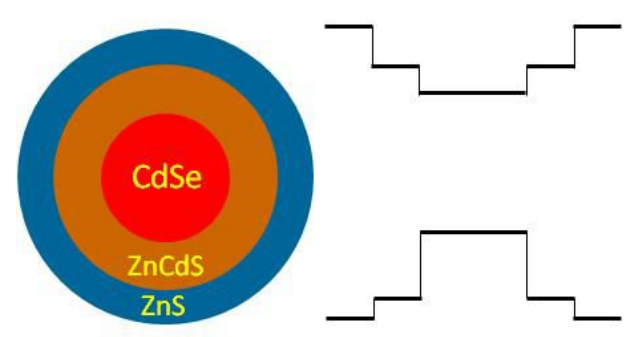

(a) CSS IQD

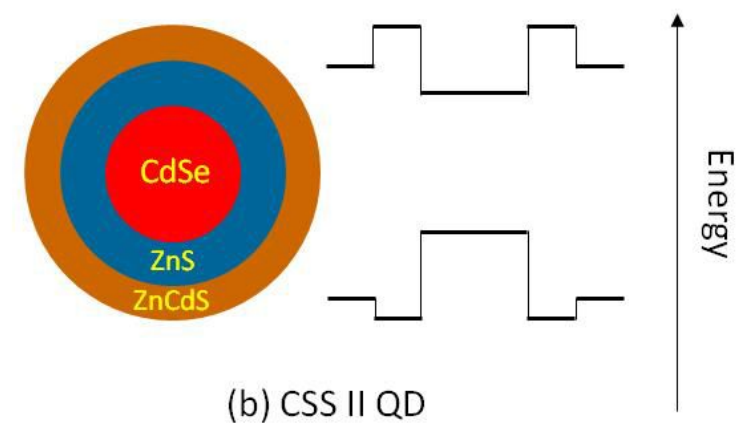

(b) CSS II QD

Fig. 2.6 Schematic cross-sections and energy band diagrams of two different CSS QDs

Similar to the CS QD synthesis, we used a modified SILAR method for the growth of two different kinds of $\mathrm{ZnCdS} / \mathrm{ZnS}$ and $\mathrm{ZnS} / \mathrm{ZnCdS}$ multishell on CdSe core QDs. CdSe core QDs (2.64 $\mathrm{nm}$ in diameter,) mixed with $18 \mathrm{~mL}$ of ODE and $6 \mathrm{~g}$ of ODA in a three-necked flask was evacuated similar as the core synthesis and then heated to $240{ }^{\circ} \mathrm{C}$ in $\mathrm{N}_{2}$ atmosphere. The zinc precursor solution $(0.1 \mathrm{M})$ was prepared by dissolving $\mathrm{ZnO}$ in $\mathrm{OA}(9.1 \mathrm{~mL})$ and $\mathrm{ODE}(25.9 \mathrm{~mL})$ at $310{ }^{\circ} \mathrm{C}$. The cadmium precursor solution $(0.1 \mathrm{M})$ was prepared by dissolving $\mathrm{CdO}(0.1541 \mathrm{~g})$ in $\mathrm{OA}(3 \mathrm{~mL})$ and ODE $(9 \mathrm{~mL})$ at $250{ }^{\circ} \mathrm{C}$. The $\mathrm{Cd}_{0.5} \mathrm{Zn}_{0.5^{-}}$precursor solution $(0.1 \mathrm{M})$ was prepared by dissolving $\mathrm{ZnO}(0.1221 \mathrm{~g})$ as well as $\mathrm{CdO}(0.1926 \mathrm{~g})$ in OA $(7.8 \mathrm{~mL})$ and ODE $(22.2 \mathrm{~mL})$ at $300{ }^{\circ} \mathrm{C}$. The sulfur precursor solution $(0.1 \mathrm{M})$ was prepared by dissolving sulfur in $\mathrm{ODE}$ at $180{ }^{\circ} \mathrm{C}$. All of the solutions were freshly prepared under $\mathrm{N}_{2}$ atmosphere. The $\mathrm{Cd}$-, $\mathrm{Zn}$-, and $\mathrm{Cd}_{0.5} \mathrm{Zn}_{0.5}$-precursor solutions were kept at about 80 ${ }^{\circ} \mathrm{C}$, while the sulfur injection solution was allowed to cool to room temperature. A similar calculation method as used in $\mathrm{CdSe} / \mathrm{ZnS}$ core/shell QDs synthesis was used to calculate the amounts of precursor solutions. The average thicknesses of one ML of $\mathrm{CdS}$ and one $\mathrm{ML}$ of $\mathrm{ZnS}$ are $0.35 \mathrm{~nm}$ and $0.31 \mathrm{~nm}$, respectively. Therefore, the thickness of one monolayer of $\mathrm{Cd}_{0.5} \mathrm{Zn}_{0.5} \mathrm{~S}$ is assumed to be $0.33 \mathrm{~nm}$. For each shell growth, a calculated amount of a given precursor solution was injected with a syringe using standard air-free procedures with an interval of $10 \mathrm{~min}$, and after the shell growth the reaction mixture was allowed to cool to room temperature. This was followed by the QD extraction step. ${ }^{[53]}$ Further purification of QDs was done by precipitating them with acetone or methanol. Highly pure QDs were obtained by repeating above purification procedure for 2-3 times. Aliquots of the solution were removed after each monolayer of shell growth with a syringe and tested for optical spectra. 

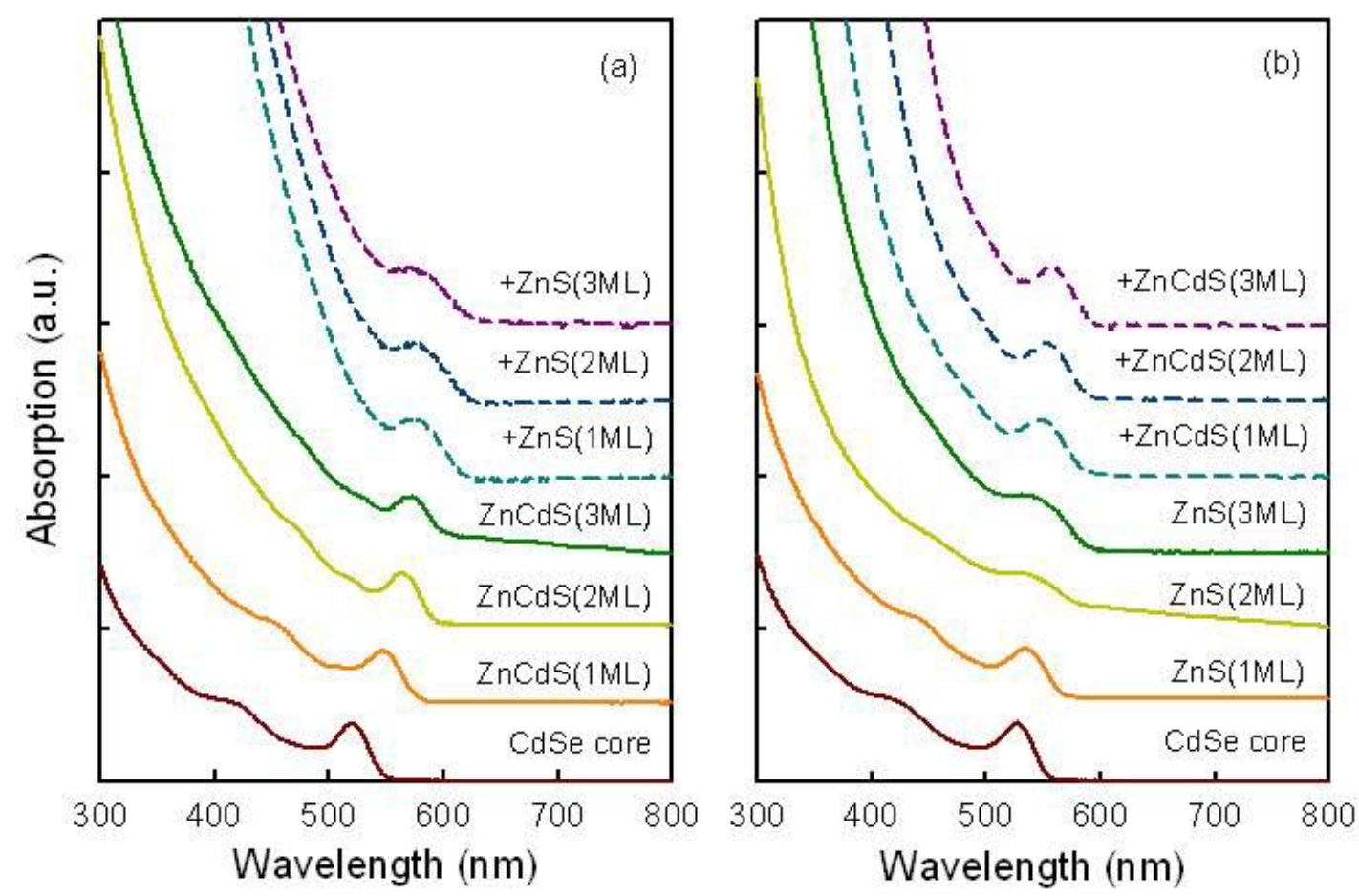

Fig. 2.7 Evolution of the absorption spectra of (a) CdSe/ZnCdS/ZnS CSS I QDs and (b)

CdSe/ZnS/ZnCdS CSS II QDs during the shell growth. The solid lines are spectra of CS QDs.

Figure 2.7 shows the evolution of the absorption spectra of the CS QDs (solid lines) and CSS QDs (dashed lines) during the shell growth. The CdSe core QDs has a first excitonic absorption peak at $521 \mathrm{~nm}$. The full width at the half maximum of the peak is $\sim 30 \mathrm{~nm}$, suggesting a narrow size distribution. As the shell growth proceeds, the peak exhibits red shift and broadening due to the penetration of the electron and/or hole wave functions into the shell layers. ${ }^{[11]}$ The red shift is more pronounced in the $\mathrm{CdSe} / \mathrm{ZnCdS}$ CS QDs than in the CdSe/ZnS CS QDs. The peak shifts by as much as $\sim 53 \mathrm{~nm}$ in the CdSe/ZnCdS (3 MLs) QDs, as compared to $22 \mathrm{~nm}$ in CdSe/ZnS (3 MLs) QDs, since ZnCdS alloying shell cannot provide potential barriers large enough to prevent the leakage of excitons into the shell as we showed in the last chapter, whereas the $\mathrm{ZnS}$ shell, whose bulk band gap is $0.6 \mathrm{eV}$ larger, can confine carriers inside the cores more efficiently. However, the CdSe/ZnS CS QDs show more significant peak broadening, especially after the growth of 2-3 MLs of ZnS. This can be attributed to a larger lattice mismatch between $\mathrm{CdSe}$ and $\mathrm{ZnS}$ (as compared to 
$\mathrm{CdSe} / \mathrm{ZnCdS}$ ), which may cause geometry irregularity or size variations. ${ }^{[54]}$ As expected, growing a ZnS outer shell around the CdSe/3 MLs ZnCdS CS QDs results in minimal further red shift, whereas growing a $\mathrm{ZnCdS}$ outer shell around the $\mathrm{CdSe} / 3$ MLs ZnS CS QDs causes a small additional red shift $(\sim 11 \mathrm{~nm})$. Interestingly, the former display significant peak broadening, indicative of degraded QD quality, whereas the latter show reduced peak broadening, evidencing the effect of strain compensation by the ZnCdS outer shell. Overall, the CSS II QDs exhibit more controllable peak wavelength and better quality than the CSS I QDs, due to more effective carrier confinement by the $\mathrm{ZnS}$ intermediate shell and more effective strain reduction by the compensating outer shell.

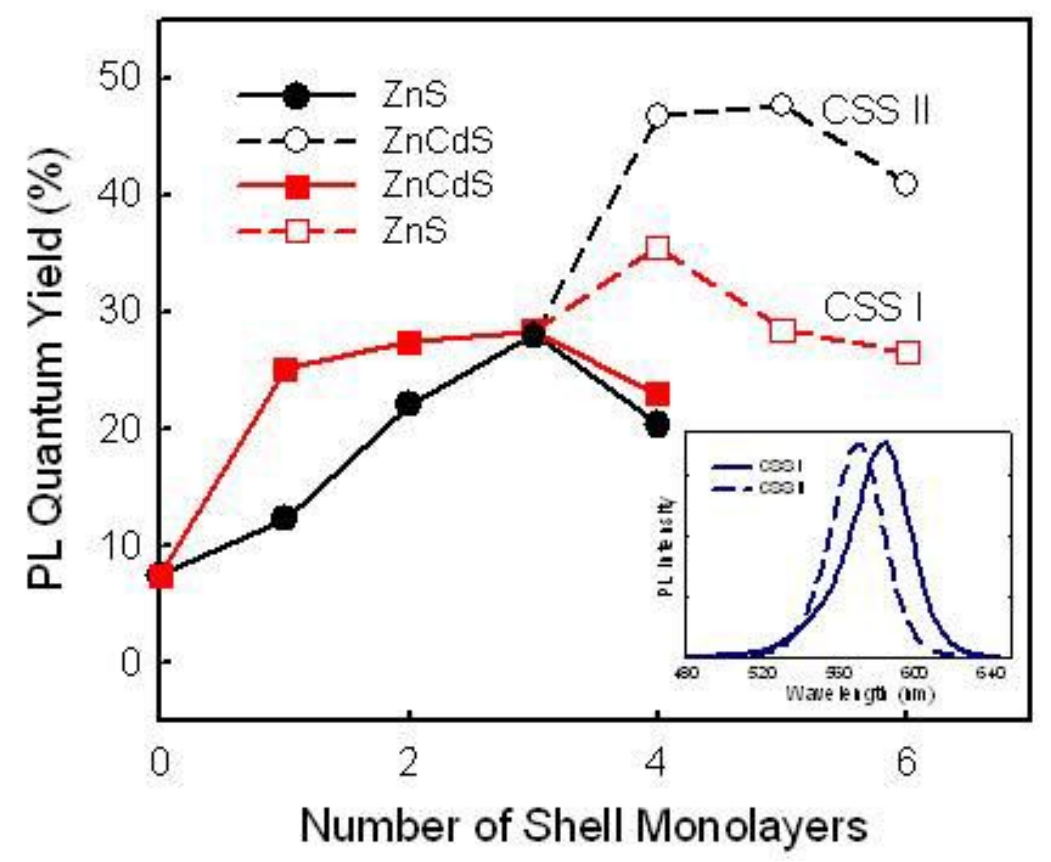

Fig. 2.8 Evolution of the PL QY of CdSe CS and CSS QDs during the shell growth. The inset shows the PL spectra of two types of CSS QDs with a normalized peak intensity

The evolution of the PL QY of the CSS QDs in solutions is shown in Figure 2.8. The typical PL spectra are shown in the inset. The QY of the CdSe core QDs is 7.5\%. The overgrowth of both $\mathrm{ZnS}$ and $\mathrm{ZnCdS}$ passivating shells leads to a dramatic improvement in the QY. It has been found that the QY of both $\mathrm{CdSe} / \mathrm{Cd}_{0.5} \mathrm{Zn}_{0.5} \mathrm{~S}$ CS QDs and CdSe/ZnS CS QDs attains a maximum value of $~ 28 \%$ after the growth of 3 
MLs of the shell material, and then decreases as the shell grows thicker. This behavior has been observed in many other CS QDs with a significant lattice mismatch between the core and shell. ${ }^{[1]}$ The initial shell growth is coherent and strain builds up in the shell as it grows thicker. When a critical thickness is reached, strain is relieved through the formation of misfit dislocations at the interface, which act as nonradiative recombination sites, deteriorating the luminescent properties of the QDs. ${ }^{[15,19]}$ As seen in Figure 2.8, further growth of a $\mathrm{ZnS}$ outer shell around the $\mathrm{CdSe} / \mathrm{Cd}_{0.5} \mathrm{Zn}_{0.5} \mathrm{~S}$ (3 MLs) CS QDs and a $\mathrm{Cd}_{0.5} \mathrm{Zn}_{0.5} \mathrm{~S}$ outer shell around the CdSe/ZnS (3 MLs) CS QDs leads to a further improvement of the PL QY. The CdSe/3 ML ZnCdS/ZnS QDs (CSS I QDs) acquire the highest QY of 36\% after adding one ML of ZnS. A thicker ZnS outer shell, however, degrades the PL QYs. The PL QY of the CdSe/3 ML ZnS/ZnCdS QDs (CSS II QDs) achieves a more remarkable improvement and peaks at $48 \%$ with a 2 ML ZnCdS outer shell. $\mathrm{Cd}_{0.5} \mathrm{Zn}_{0.5} \mathrm{~S}$ has a smaller lattice mismatch $(\sim 8 \%)$ with $\mathrm{CdSe}$ compared to $\mathrm{ZnS}$. In the CSS I structure, it functions as a lattice adaptor and strain-reducing layer, ${ }^{[20]}$ whereas in the CSS II structure, it partially compensates the strain in the $\mathrm{ZnS}$ shell, giving rise to a strain-compensated bilayer shell structure. So strain relief can be achieved in both CSS QDs, but by different mechanisms. The results in Figure 2.8 suggest that the strain compensation strategy is more effective in improving the quality of the QDs. In this special case, the CSS II QDs take the benefits of excellent exciton confinement by a $\mathrm{ZnS}$ intermediate shell and strain compensation by a ZnCdS outer shell. As seen in Figure 2.7, another advantage of the CSS II structure is a much smaller peak red shift resulting from the shell growth.

The crystallinity of the CSS QDs was examined by powder X-ray diffraction (XRD) on a Bruker D8 Discover X-Ray Diffractometer using $\mathrm{Cu}$ a radiation ( $\mathrm{nm}$ ). A representative XRD pattern for both CSS I and CSS II QDs are shown in Figure 2.9(a) and (b). Three distinct diffraction peaks were observed at $2 \mathrm{~h}$ values of 25.8 , 43.3, and 51.2 degree, corresponding to the (111), (220), and (311) crystalline planes of cubic CdSe, respectively. The broadening of the diffraction peaks is expected due to the nanocrystalline nature of the core and shell. 

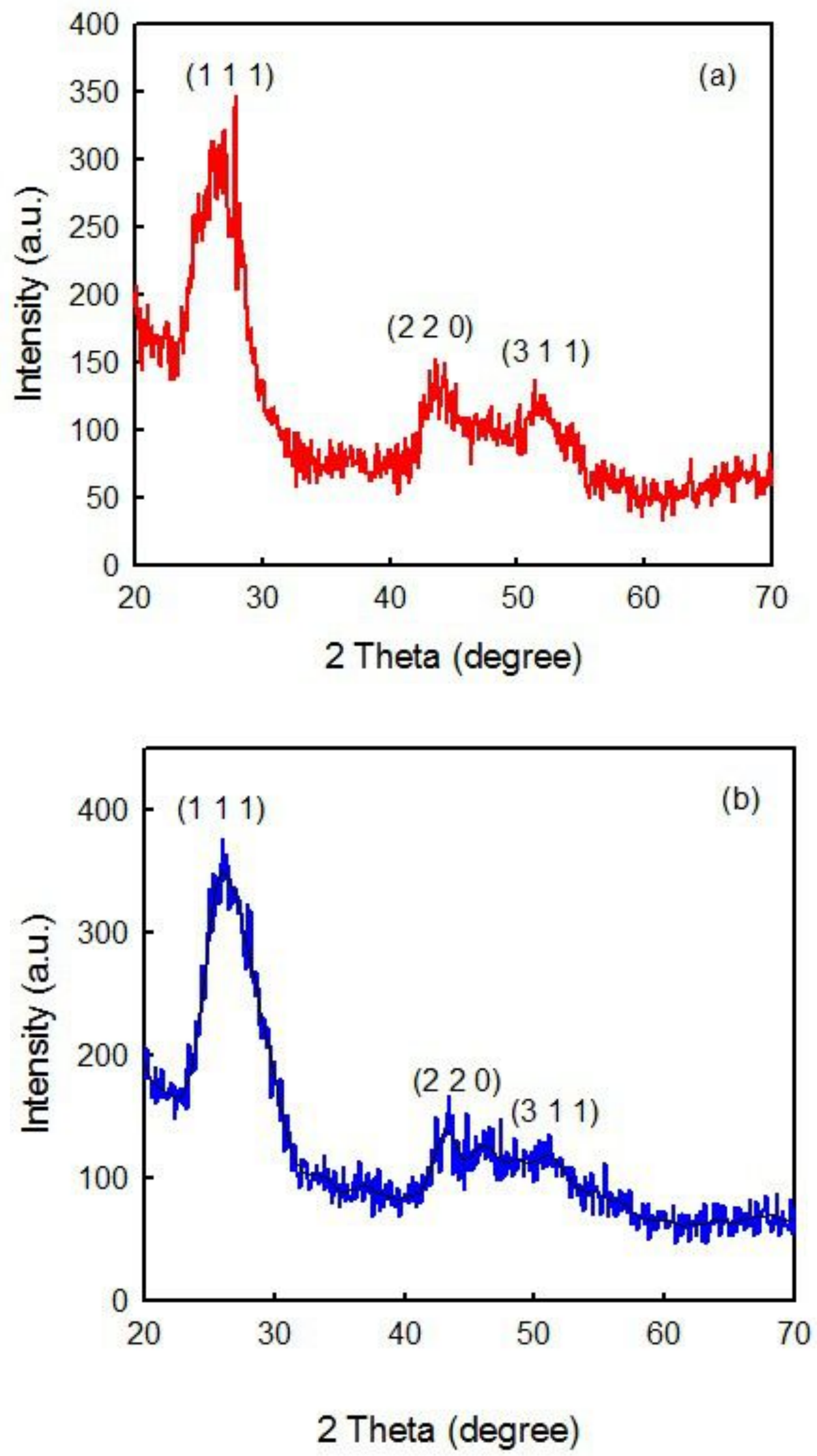

Fig. 2.9 Powder XRD Spectra of (a) CdSe/ZnS/ZnCdS QDs (b) CdSe/ZnCdS/ZnS QDs 


\subsection{Demonstration of High-Efficiency LEDs based on strain- compensated QDs}

\subsubsection{Structure Optimization}

The QD-LEDs were fabricated on glass substrates with pre-patterned ITO (sheet resistance $\sim 15 \Omega / \square$ ). The substrates were sequentially cleaned in acetone, ethanol, and de-ionized water, and exposed to oxygen plasma for 5 min. A $40 \mathrm{~nm}$ poly(3,4-ethylenedioxythiophene)poly(styrenesulfonate) (PEDOT:PSS) (Aldrich 560596) layer was first spin-coated at a spin rate of $5000 \mathrm{rpm}$ for $30 \mathrm{~s}$ and dried at $150{ }^{\circ} \mathrm{C}$ under $\mathrm{N}_{2}$ for $10 \mathrm{~min}$. A $40 \mathrm{~nm}$ poly-(N,N'-bis(4-butylphenyl)N,N'-bis(phenyl)benzidine) (poly-TPD) (ADS 254BE) layer was then spin-coated from a chloroform solution $(6 \mathrm{mg} / \mathrm{mL})$ on top of the PEDOT:PSS layer at $2500 \mathrm{rpm}$ for $30 \mathrm{~s}$, followed by annealing at $110^{\circ} \mathrm{C}$ in $\mathrm{N}_{2}$ for $30 \mathrm{~min}$. CSSQDs were then deposited on the surface of poly-TPD by spin-coating from a hexane solution at a rate of $3000 \mathrm{rpm}$ for $1 \mathrm{~min}$. The samples were then transferred from a glovebox to a high-vacuum thermal evaporation system. A multilayer structure was deposited onto the QD layer through a shadow mask by evaporation deposition at rates of $\sim 0.1 \mathrm{~nm} / \mathrm{s}$ under $3 \times 10^{-7}$ Torr. The structure consisted of a $40 \mathrm{~nm}$ TPBI electron injection layer (ETL), a $0.5 \mathrm{~nm} \mathrm{LiF}$ cathode buffer layer and $120 \mathrm{~nm}$, and an Al cathode. To optimize the QD layer thickness, the QD emitting layers prepared from solutions of different QD concentrations from 1-8 $\mathrm{mg} / \mathrm{mL}$. For 1, 3, 5, $8 \mathrm{mg} / \mathrm{ml}$ solutions, the corresponding QD layer thickness was estimated to be 0.5 MLs, 1.6 MLs, 3 MLs and 5 MLs, respectively, based on the measured optical properties of the QD films. ${ }^{[56]}$ All the QD-LEDs had an emitting area of $\sim 0.1 \mathrm{~cm}^{2}$. Figure 2.11 shows the Trovato evaporation system integrated with MBraun glovebox for fabrication process of QD-LEDs.

The QD-LEDs were then encapsulated with glass lids in $\mathrm{N}_{2}$ and characterized in air at room temperature. The electrical characteristics were measured using an Agilent 4156C semiconductor analyzer. The EL spectra were recorded as a function of 
injection current density in the range of $1-100 \mathrm{~mA} / \mathrm{cm}^{2}$ using a silicon photodiode-array fiber-optic spectrometer. To calculate EQE, the QD-LEDs were placed directly on the surface of a calibrated silicon photodetector and all emitted photons from the glass side were captured.

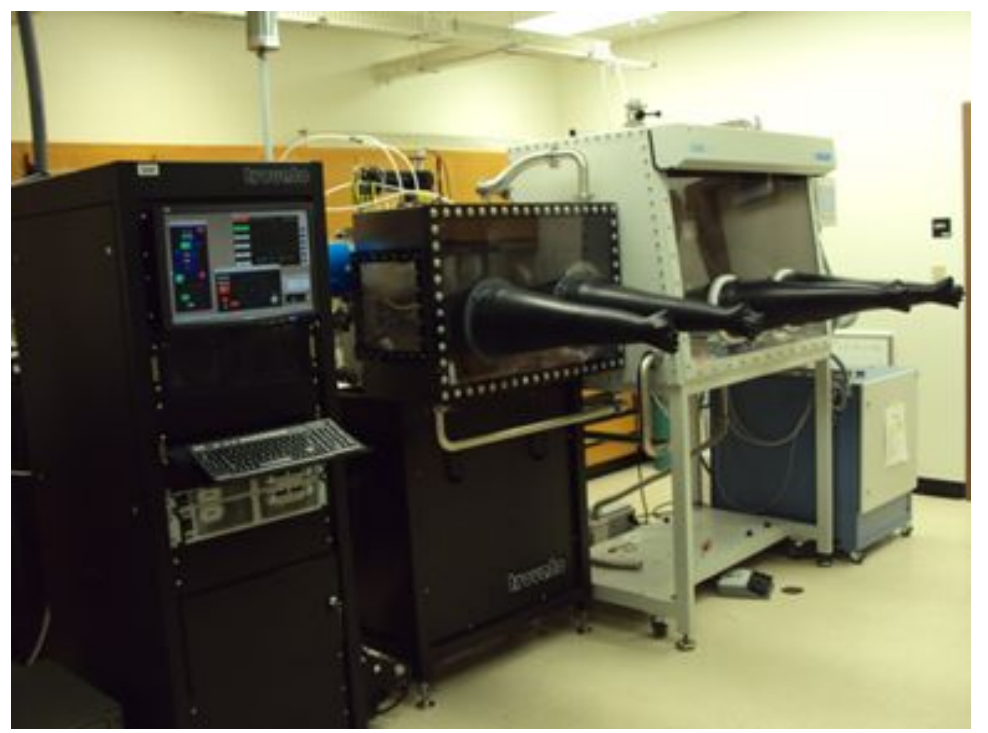

Fig.2.11 Trovato evaporation system integrated with MBraun glovebox for fabrication of

\section{QD-LEDs}

The proposed CSS QD-LEDs structure and energy diagram are schematically shown in Figure 2.12. PEDOT:PSS layer was used as hole injection layer (HIL) and the poly-TPD was chosen as the HTL because it possesses an intrinsic resistance to nonpolar organic solvents and a high hole mobility with effective electron blocking properties. Two different kinds of CSS QDs were used as the emission layer, followed by TPBI ETL deposition.
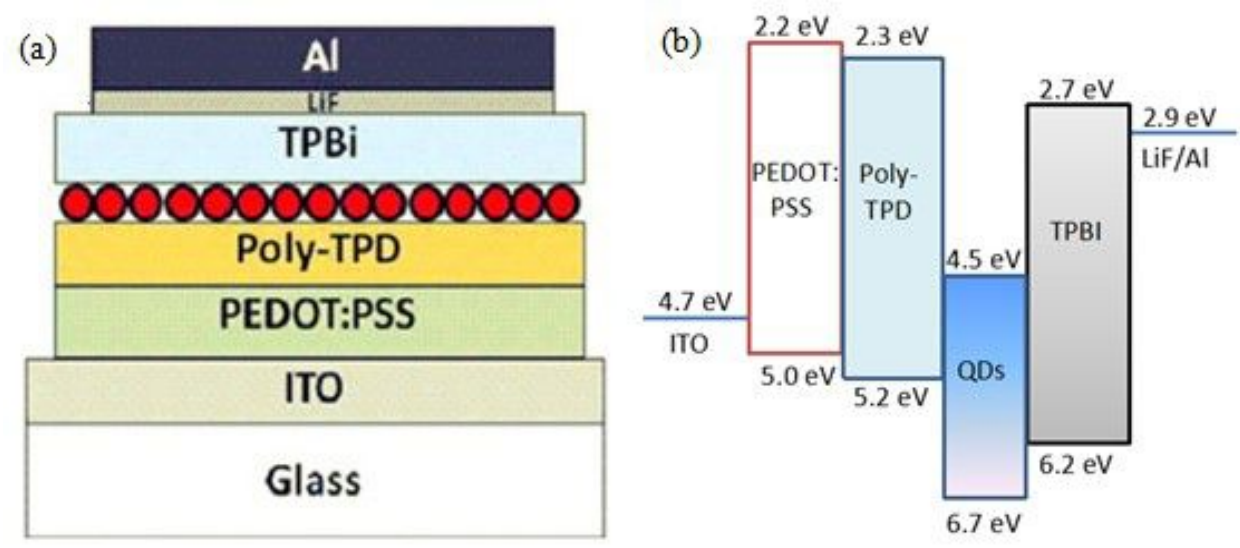

Fig. 2.12 (a) Layer structure of a CSS QD-LED (b) The energy diagram of the QD-LED 
To optimize the QD layer thickness, CSS IIQDs solutions with different concentrations (1-8 mg/mL) were prepared and spin coated on the poly-TPD layer, while all other experimental conditions in the test devices were kept constant. Figure 2.13 (a) shows the EL spectra of yellow QD-LEDs prepared from 1, 3, 5 and 8 $\mathrm{mg} / \mathrm{mL}$ QD dispersions in hexane. The QD-LED prepared from a $3 \mathrm{mg} / \mathrm{ml}$ solution exhibits the highest light intensity at the same current density. The corresponding layer thickness was estimated to be 1.6 MLs based on the measured optical properties of the QD films. Figure 2.13 (b) demonstrates the normalized EL spectra of green QD-LEDs prepared from $3 \mathrm{mg} / \mathrm{mL}$ QD solution. Pure QDs emitting peak without any organic emitting peaks is realized from $0.1 \mathrm{~mA}$ to $10 \mathrm{~mA}$.
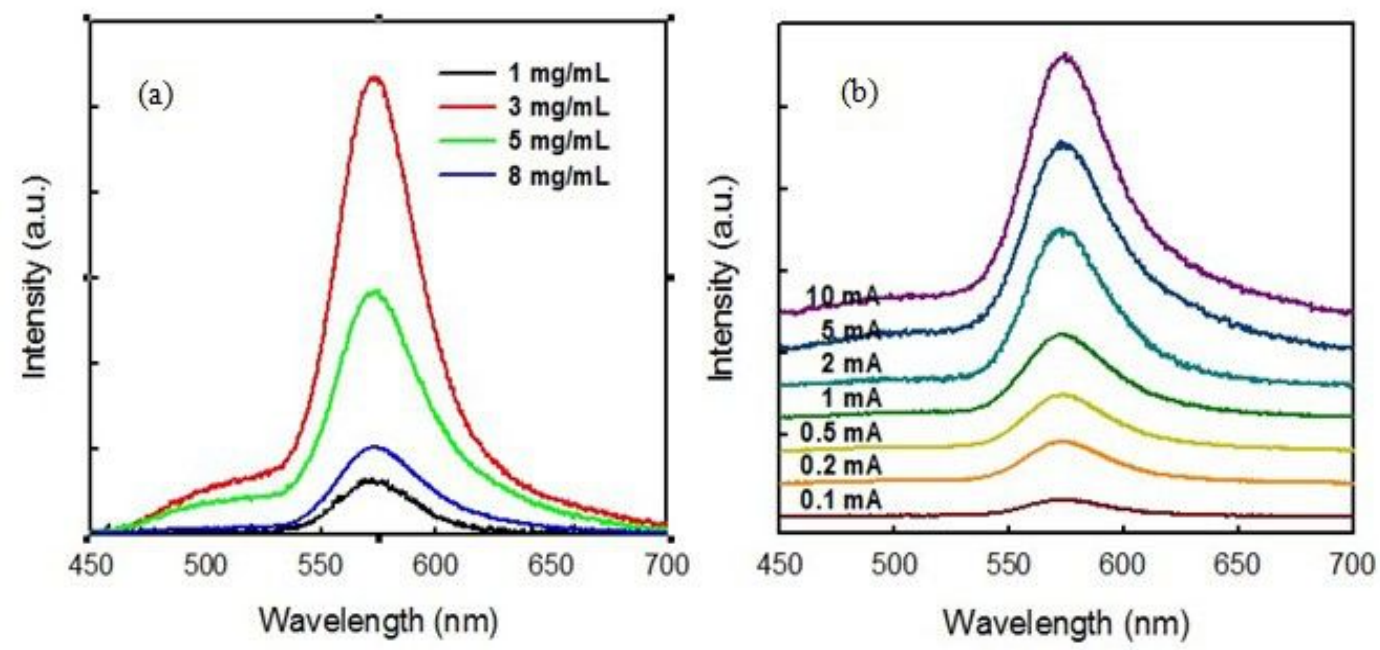

Fig. 2.13 (a) EL spectra of CSS II QD-LEDs prepared from 1-8 mg/mL QD solutions recording at 1 mA. (b) Normalized EL spectra of CSS II QD-LEDs prepared from $3 \mathrm{mg} / \mathrm{mL}$ QD solution

The QD-LED prepared from a $3 \mathrm{mg} / \mathrm{mL}$ solution shows a moderate turn-on voltage of $\sim 4 \mathrm{~V}$ (Figure 2.14). Both the turn-on voltage and series resistance of the QD-LEDs increase as the thickness of the QD layer increases. The QD-LED with QDs spin coated from a $1 \mathrm{mg} / \mathrm{mL}$ solution has the lowest forward voltage, but its EL is 5 times lower, presumably due to a partial surface coverage by less than one monolayer of QDs. 


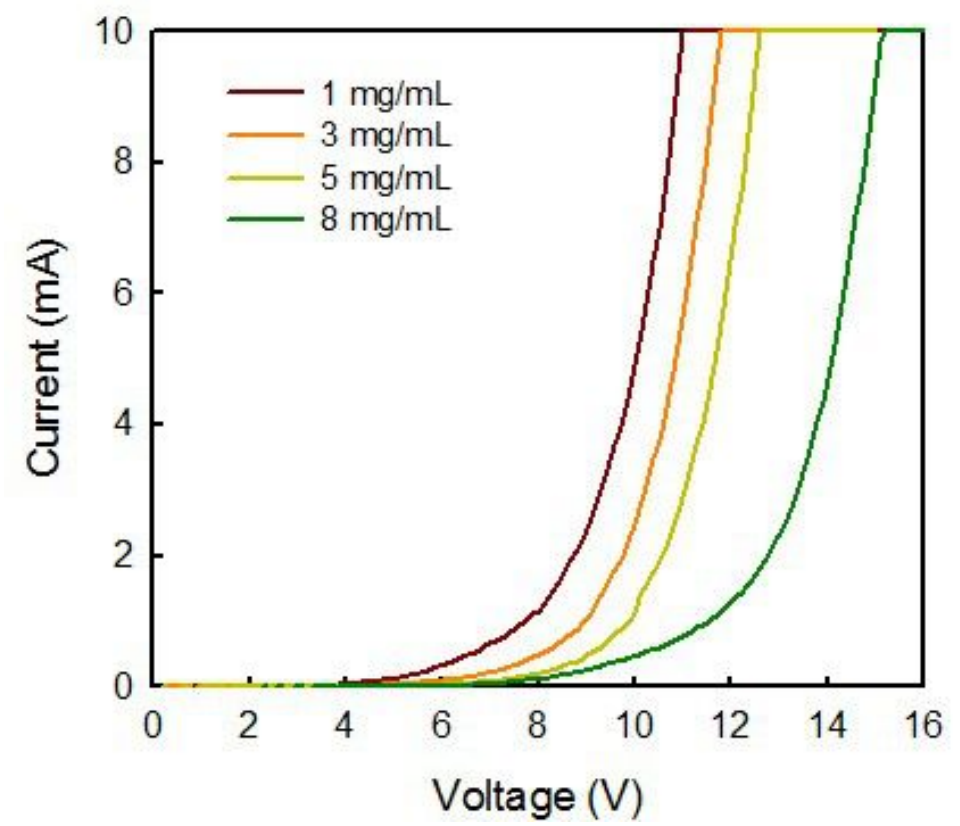

Fig. 2.14 I-V Characteristics of CSS II QD-LEDs with different concentration of QD solutions

\subsubsection{Fabrication and Characterization of CSS QD-LEDs}

For the fabrication of QD-LEDs, CdSe/2 MLs ZnCdS/2 MLs ZnS (CSS I) and CdSe/2 MLs ZnS /2 MLs ZnCdS (CSS II) QDs were synthesized using the same procedure as we discussed in section 2.1.3, and subjected to a multistep precipitation and redissolvation process of purification, respectively. The devices were fabricated by spin-coating of polymer and QD layers on indium-tin-oxide/glass substrates, followed by evaporation deposition of organic and metal films. The QD-LED structure (Figure 2.15) consisted of a 40nm PEDOT:PSS HIL, a $40 \mathrm{~nm}$ poly-TPD HTL, a CSS QD emitting layer (EML), and a 40nm TPBi ETL. LiF/Al (0.5/120 nm) was deposited on top of the TPBi layer as the cathode, whose overlap with the ITO anode defines an emitting area of $0.1 \mathrm{~cm}^{2}$. To optimize the EML thickness, the QDs were deposited by spin coating from 1-4 mg/mL QD/hexane solutions, which resulted in estimated QD coverages of 0.5-2.1 MLs as deduced from measurements of the optical density of the QD films. The QD-LEDs were then encapsulated with glass lids in $\mathrm{N}_{2}$ and characterized in air at room temperature. 


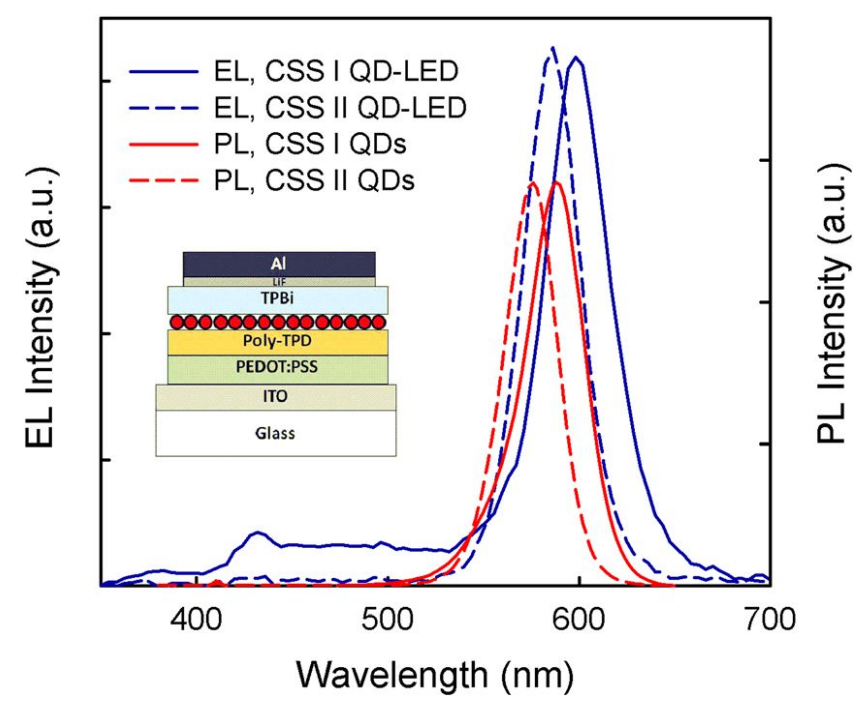

Fig. 2.15 EL spectra of CSS I and CSS II QD-LEDs at $20 \mathrm{~mA} / \mathrm{cm}^{2}$, along with the PL spectra of the QDs measured in hexane solutions. The EL and PL peak intensities are normalized.

The LED containing QDs ( 1.2 MLs) prepared from $2 \mathrm{mg} / \mathrm{mL}$ solutions had the best performance. Figure 2.16 shows their EL spectra recorded at $0.2-20 \mathrm{~mA} / \mathrm{cm}^{2}$. In both LEDs, the EL originates from QDs only and no parasitic emission from the organic layers is observed. While the CSS II QD-LED emits purely yellow light with a peak at $583 \mathrm{~nm}$, the EL spectrum of the CSS I QD-LED comprises weak broad emission in the blue-green wavelength region on the high-energy side of the main 595 $\mathrm{nm}$ peak. Such side emission, which appears to be weaker in the low injection regime, is absent in the PL of purified CSS I QDs. We believe that it arises from exciton recombination in the $\mathrm{ZnCdS}$ intermediate shell. The discrepancy between the EL and PL may be explained as follows. The optical excitation intensity of PL by a broadband lamp through a monochromator is low, so only a small number of excitons are generated, which are mainly confined and recombine in the CdSe core. However, under current injection, a much higher density of excitons may be created in the core through direct injection and energy transfer, ${ }^{[57]}$ which cannot be well confined by the $\mathrm{ZnCdS}$ intermediate shell. A small portion of the excitons may overflow into $\mathrm{ZnCdS}$ and undergo radiative decay. The broad band emission suggests that the $\mathrm{ZnCdS}$ shell may consist of Zn-rich and Cd-rich phases. Such compositional in homogeneity proves that it is difficult to form a completely random $\mathrm{ZnCdS}$ alloy with a uniform composition due a large lattice mismatch between $\mathrm{ZnS}$ and $\mathrm{CdS}$. 

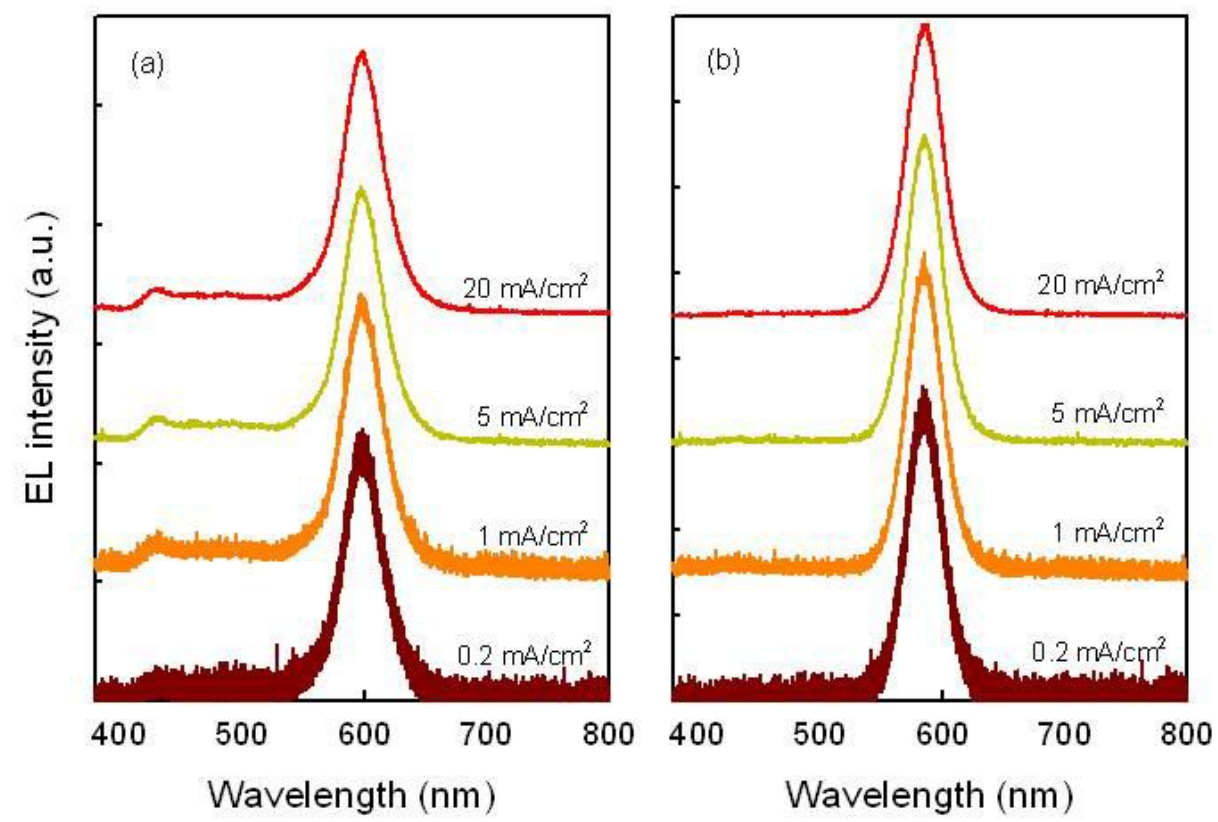

Fig. 2.16 EL spectra of (a) CSS I and (b) CSS II QD-LEDs at increasing current density. The EL peak intensities are normalized, and the spectra are shifted in the y-direction for clarity.

Figure 2.17 shows the forward current-voltage (I-V) characteristics of the two QD-LEDs. Both devices turn on at $4 \mathrm{~V}$. Their operational voltages at $20 \mathrm{~mA} / \mathrm{cm}^{2}$ and series resistances are also comparable. Also displayed in Figure 2.17 are the I-V curves of LEDs with QDs prepared from $4 \mathrm{mg} / \mathrm{mL}$ solutions ( 2.1 ML QDs). At 20 $\mathrm{mA} / \mathrm{cm}^{2}$, both LEDs have a higher voltage than their counterparts with QDs prepared from $2 \mathrm{mg} / \mathrm{ml}$ solutions, by $2.6 \mathrm{~V}$ and $2.1 \mathrm{~V}$, respectively. This is expected since LEDs with a thick QD EML suffer more from poor charge transport between QDs capped with insulating organic ligands. ${ }^{[58]}$ 


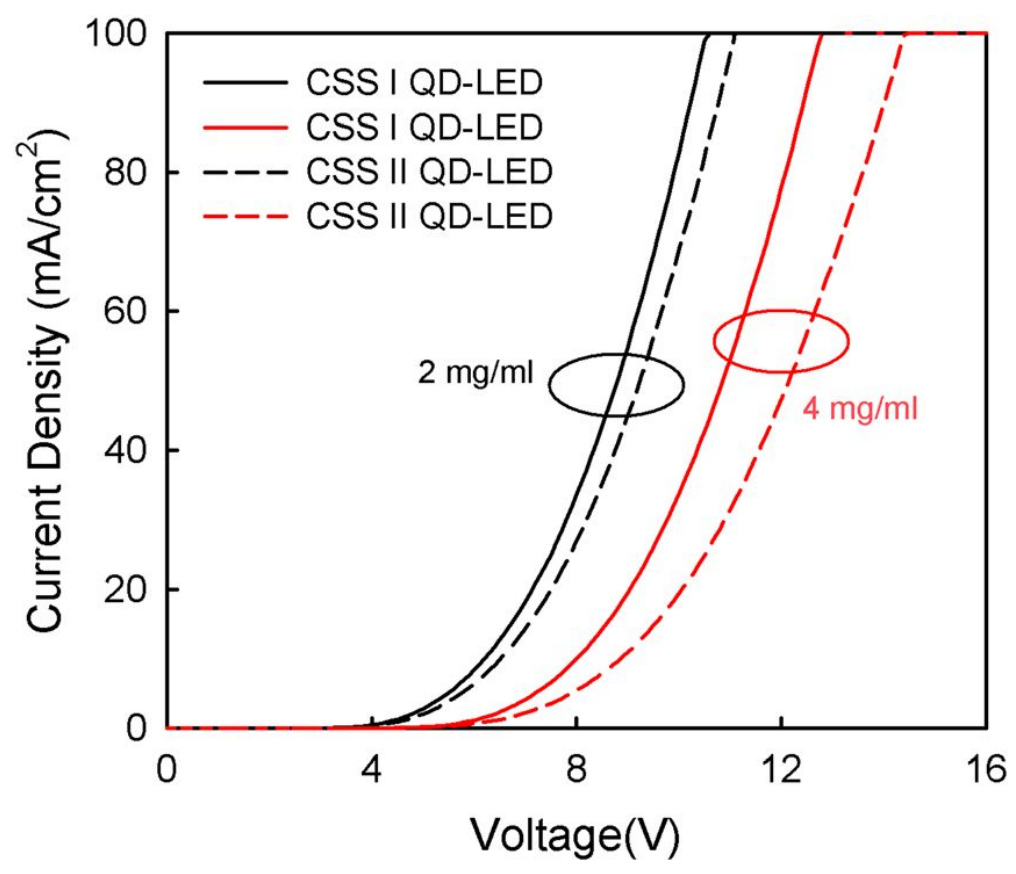

Fig. 2.17 Forward I-V characteristics of CSS I and CSS II QD-LEDs with the QD EML prepared from $2 \mathrm{mg} / \mathrm{ml}$ and $4 \mathrm{mg} / \mathrm{ml}$ QD solutions.

Figure 2.18 compares the luminance-current (L-I) characteristics of the LEDs based on CSS I and CSS II QDs deposited from $2 \mathrm{mg} / \mathrm{ml}$ solutions. The CSS II QD-LED is $28 \%$ brighter than the CSS I QD-LED at $20 \mathrm{~mA} / \mathrm{cm}^{2}$. This result is consistent with the optical characterization of the QDs conducted prior to device fabrication, which showed the PL QY of the CSS II QDs was $40 \%$ higher than that of CSS I QDs. Meanwhile, the correlation between the PL and EL also suggests that the rates of exciton generation, by charge injection or energy transfer, in the EML of both LEDs are similar despite the different shell structures of the QDs. At $20 \mathrm{~mA} / \mathrm{cm}^{2}$, the CSS II QD-LED has a luminance of $556 \mathrm{~cd} / \mathrm{m}^{2}$, a current efficiency of $2.8 \mathrm{~cd} / \mathrm{A}$, and an EQE of $0.65 \%$. Its brightness level reaches $2112 \mathrm{~cd} / \mathrm{m}^{2}$ at $100 \mathrm{~mA} / \mathrm{cm}^{2}$. As seen from Figure 2.18, compared to LEDs fabricated using $4 \mathrm{mg} / \mathrm{ml}$ QD solutions, the LEDs with QDs prepared from $2 \mathrm{mg} / \mathrm{ml}$ solutions are $32 \%$ brighter at $20 \mathrm{~mA} / \mathrm{cm}^{2}$. 


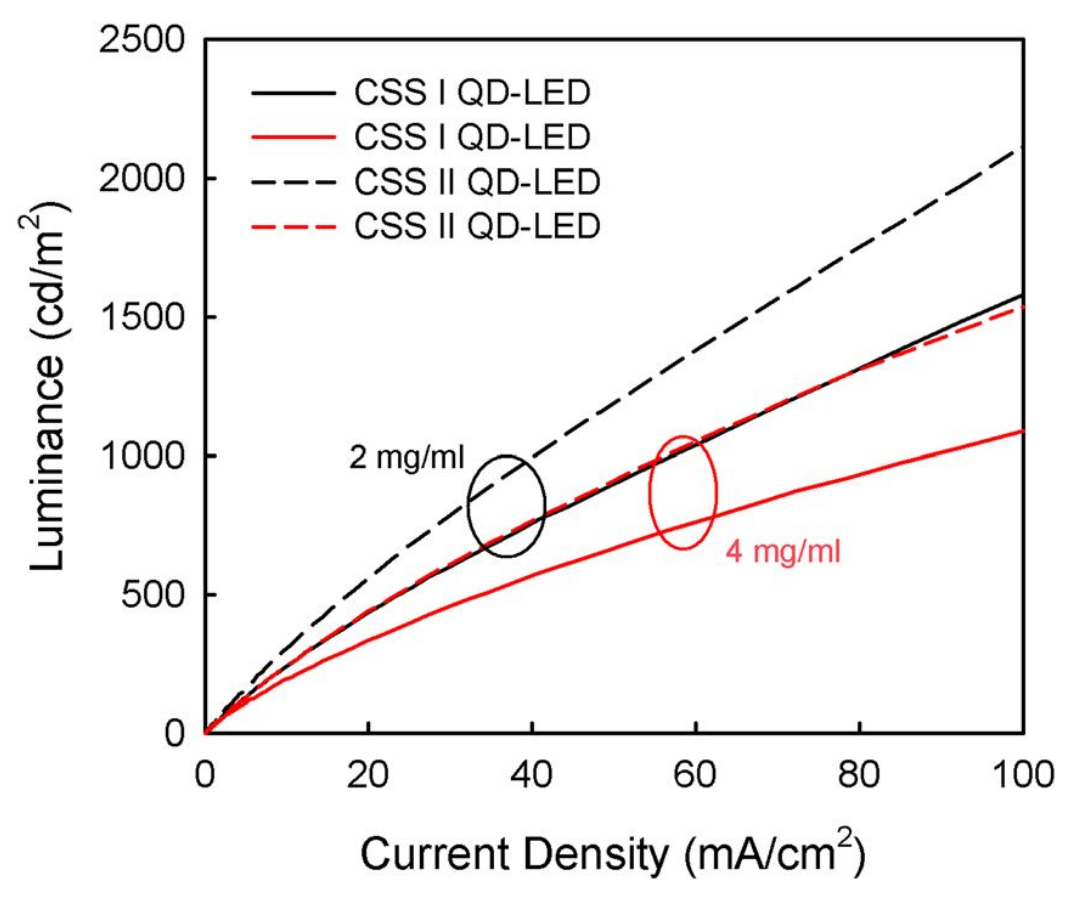

Fig. 2.18 L-I characteristics of CSS I and CSS II QD-LEDs with the QD EML prepared from $2 \mathrm{mg} / \mathrm{ml}$ and $4 \mathrm{mg} / \mathrm{ml}$ QD solutions.

\subsection{Conclusions}

In summary, we have investigated the effects of two different strain-relief bilayer shell structures on the luminescence of colloidal CdSe QDs. A ZnCdS strain-compensating outer shell has proven to be more effective than a $\mathrm{ZnCdS}$ strain-reducing intermediate shell in improving the PL QY of CdSe/ZnS QDs. The resulting CdSe/ZnS/ZnCdS CSS QDs were $40 \%$ more efficient than the $\mathrm{CdSe} / \mathrm{ZnCdS} / \mathrm{ZnS}$ QDs, and have a more controllable peak wavelength. LEDs based on the former QDs exhibited 28\% higher brightness and purer EL spectra. These results suggest that nanocrystal QDs of high crystalline and optical quality can be obtained through strain-engineering in their nanoshell structures. 


\section{Chapter 3 Synthesis of CdSe Quantum-Dot}

\section{Quantum-Well nanocrystals for blue light emission}

So far, efficient red and green LEDs have been realized based on CdSe QDs with various dot sizes, but QD-LEDs emitting efficient blue light have yet to be developed due to a lack of appropriate QD materials. ${ }^{[59,}{ }^{60]}$ In principle, blue light emission $\sim 470$ $\mathrm{nm}$ needed for flat panel display applications may be obtained from CdSe QDs smaller than $2 \mathrm{~nm} \cdot{ }^{[53]}$ However, such small QDs with narrow size distributions and good quantum efficiencies are difficult to synthesize. Overcoating such small QDs with a wider bandgap passivating shell, which is necessary to improve the QD luminescent efficiency and stability, is also difficult. In addition, small QDs have a small absorption cross section, which leads to slow excitonic energy transfer into the QDs. All these factors make CdSe core QDs a poor choice for achieving efficient blue luminescence.

The recent development of colloidal nanocrystals with diverse internal heterostructures provides an alternative means for tuning the optical properties of nanoparticles based on one particular material. ${ }^{[61,26]}$ Among those unique nanocrystal heterostructures are quantum dot quantum wells (QDQWs), which are synthesized with a core of a large bandgap material followed by an intermediate shell of a smaller bandgap material and an outer shell of the same large bandgap material as the core. In analogy with planar QW structures, the intermediate shell acts as a QW, whereas the core and outer shell function as two cladding layers. These nanocrystal heterostructures are attractive since they allow independent control of the bandgap of the emitting layer and the overall nanocrystal size. For example, narrow blue light-emitting QWs may be incorporated into nanocrystals with a relatively large size which facilitates synthesis and processing. ${ }^{[26]}$ Unlike expensive vacuum epitaxy techniques used to produce conventional planar semiconductor QWs, the chemical colloidal synthesis provides a low-cost pathway to preparing unique 2Dnanostructures for various optical applications. 
In this chapter, we discuss the synthesis and characterization of $\mathrm{CdS} / \mathrm{CdSe} / \mathrm{ZnS}$ QDQWs, in an effort to realize efficient blue light emission from CdSe nanocrystals. The CdS core is used instead of a $\mathrm{ZnS}$ core because it has a smaller lattice mismatch with $\mathrm{CdSe}(\sim 3.7 \%$ compared to $12 \%$ for $\mathrm{ZnS} / \mathrm{CdSe})$, thus providing a more desirable template for the QW growth. Meanwhile, a ZnS outer cladding layer is used since it provides effective passivation and carrier confinement due to its wide bandgap. LEDs based on QDQW structures are developed and demonstrated as well.

\subsection{Synthesis and Characterization of CdSe Quantum-Dot Quantum-Well (QDQW) nanocrystals (NCs)}

\subsubsection{Synthesis of CdS core QDs}

TOPO/ODA-capped CdS core QDs were synthesized using standard hot injection method similar to that for CdSe core QDs. For a typical reaction, a mixture of $1 \mathrm{mmol}$ of $\mathrm{CdO}$ powder, $1.27 \mathrm{~mL} \mathrm{OA}$, and $15 \mathrm{~mL}$ ODE in a $100 \mathrm{~mL}$ three-neck flask was evacuated for $20 \mathrm{~min}$ at room temperature and $10 \mathrm{~min}$ at $100^{\circ} \mathrm{C}$, and then heated to $250^{\circ}$ Cunder $\mathrm{N}_{2}$-flow to form an optically clear solution. A mixture of 1 mmol of S powder, and $5 \mathrm{~mL}$ ODE in a $100 \mathrm{~mL}$ three-neck flask was evacuated for 20 min at room temperature and $10 \mathrm{~min}$ at $100^{\circ} \mathrm{C}$, and heated to $150^{\circ} \mathrm{C}$ under $\mathrm{N}_{2}$-flow to form an optically clear solution. The solution was then allowed to cool to room temperature, and a mixture of $3.6 \mathrm{~g}$ ODA and $1.2 \mathrm{~g}$ TOPO was added to the Cd solution. The mixture was then evacuated for $20 \mathrm{~min}$ at room temperature and $10 \mathrm{~min}$ at $100^{\circ} \mathrm{C}$, and reheated to $260^{\circ} \mathrm{C}$ under $\mathrm{N}_{2}$-flow. At this temperature, a solution containing $0.032 \mathrm{~g}$ of $\mathrm{S}$ dissolved in $5 \mathrm{~mL}$ of ODE was injected into the reaction flask. The following equation (2) shows the reaction mechanism following the injection.

The mixture was then kept at $260^{\circ} \mathrm{C}$ for $\mathrm{CdS}$ core QDs nucleation, and aliquots of the solution were removed after certain time of growth with a syringe and tested for optical spectra. The heating mantle was then removed and the mixture was quickly 
cooled by DI water bath to $100^{\circ} \mathrm{C}$, followed by a precipitation of the nanocrystals. After centrifugation and decantation, the particles were dispersed in hexane for further processing.

Figure 3.1 displays the temporal evolution of the absorption spectra of the CdS QDs, which were produced at $260{ }^{\circ} \mathrm{C}$ through the reaction of $\mathrm{CdO}-\mathrm{OA}$ in ODA-TOPO-ODE with the injected sulfur dissolved in ODE. During a $1200 \mathrm{sec}$ growth progress at the reaction temperature, the first excitonic peak of the obtained CdSe QDs gradually shift to longer wavelength from $406 \mathrm{~nm}$ to $416 \mathrm{~nm}$ due to the increased particle size. After $1200 \mathrm{sec}$ growth, the original sharp excitonic absorption peak is still retained. The sharp feature of the multiple excitonic absorption peaks demonstrates that the size of the resulting CdS QDs is nearly monodispersed with a narrow size distribution.

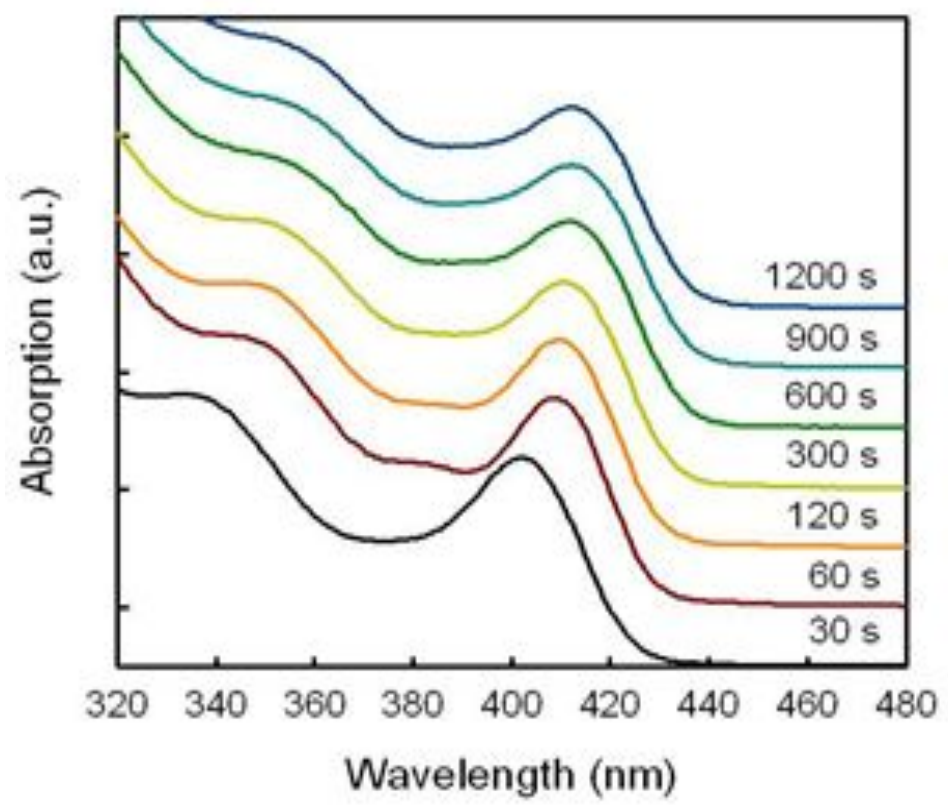

Fig. 3.1 Temporal evolution of absorption spectra of CdS QDs samples taken during the growth reaction at $260{ }^{\circ} \mathrm{C}$

An empirical function has been proposed to correlate the size of CdS QDs with the position of the first excitonic peak in its absorption spectra as shown below. ${ }^{[53]}$ $\mathrm{D}=\left(-6.6521 \times 10^{-8}\right) \lambda^{3}+\left(1.9557 \times 10^{-4}\right) \lambda^{2}-\left(9.2352 \times 10^{-2}\right) \lambda+(13.29)$ In the above function, $\mathrm{D}(\mathrm{nm})$ is the diameter of CdS QDs, and $\lambda(\mathrm{nm})$ is the wavelength of the first excitonic absorption peak. Based on this function, the evolution of the particle size during growth of the $\mathrm{CdS}$ QDs at $260{ }^{\circ} \mathrm{C}$ can be 
calculated as shown in Figure.3.2. The growth rate decreased gradually and the growth almost terminated when the particles diameter reached $\sim 3.9 \mathrm{~nm}$.

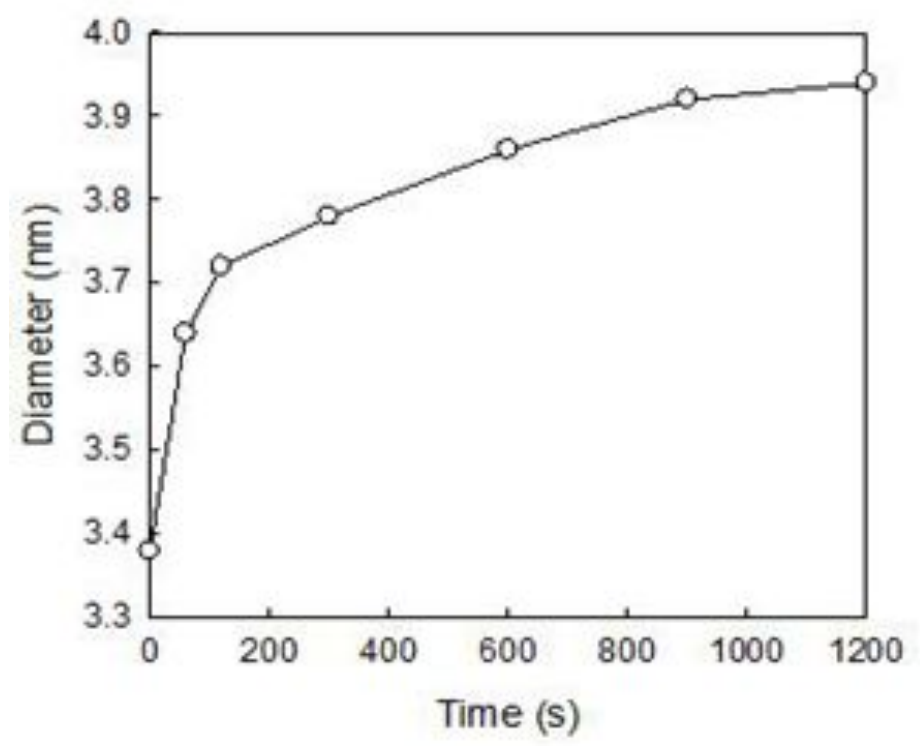

Fig. 3.2 Temporal dependence of the particle size during the growth of CdS QDs at $260^{\circ} \mathrm{C}$

The absorption spectrum as well as the PL spectrum of CdS QDs with $\sim 3.6$ $\mathrm{nm}$ in diameter is shown in Figure 3.3. The first excitonic absorption peak occurs at $406 \mathrm{~nm}$ with a corresponding PL emission at $428 \mathrm{~nm}$. The presence of a higher-order excitonic absorption peak and the narrow FWHM $(\sim 20 \mathrm{~nm})$ of the PL peak corroborates that the as-prepared CdS core QDs have a very narrow size distribution and uniform size. The PL spectrum also comprise a strong and broad band centered around $540 \mathrm{~nm}$, which can be attributed to excitonic recombination at surface states of the CdS QDs.

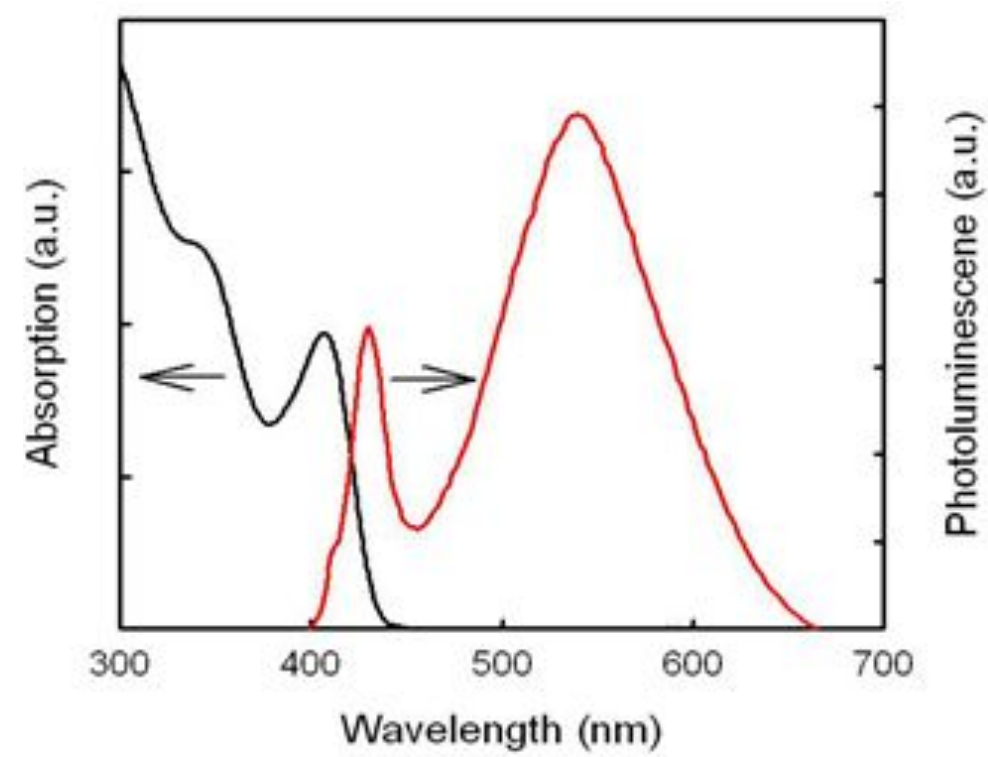


Fig. 3.3 Temporal dependence of the particle size during the growth of CdS QDs at $260{ }^{\circ} \mathrm{C}$

\subsubsection{Growth of QDQW structure}

To synthesize QDQW nanocrystals as shown in Figure 3.4, we used a modified SILAR method similar to that used previously for CSS QDs synthesis, to grow a $\mathrm{CdSe} / \mathrm{ZnS}$ bilayer shell around the CdS core QDs. The CdS core QDs () were prepared using the similar recipe described earlier and mixed with $18 \mathrm{~mL}$ of ODE and $6 \mathrm{~g}$ of ODA in a three-necked flask. The zinc precursor solution $(0.05 \mathrm{M})$ was prepared by dissolving $\mathrm{ZnO}(0.1017 \mathrm{~g})$ in oleic acid $(3.20 \mathrm{~mL})$ and $\mathrm{ODE}(21.8 \mathrm{~mL})$ at $320^{\circ} \mathrm{C}$. The cadmium precursor solution $(0.1 \mathrm{M})$ was prepared by dissolving $\mathrm{CdO}$ $(0.1602 \mathrm{~g})$ in oleic acid $(3.20 \mathrm{~mL})$ and $\mathrm{ODE}(21.8 \mathrm{~mL})$ at $300{ }^{\circ} \mathrm{C}$. The sulfur precursor solution $(0.05 \mathrm{M})$ was prepared by dissolving sulfur $(0.080 \mathrm{~g})$ in ODE (43.6 $\mathrm{mL})$ at $150{ }^{\circ} \mathrm{C}$. The selenium precursor solution $(0.05 \mathrm{M})$ was prepared by dissolving selenium $(0.1975 \mathrm{~g})$ in $\mathrm{ODE}(43.6 \mathrm{~mL})$ at $150{ }^{\circ} \mathrm{C}$. All of the solutions were freshly prepared under $\mathrm{N}_{2}$-atmosphere. The $\mathrm{Cd}$ - and $\mathrm{Zn}$ - precursor solutions were kept at about $100{ }^{\circ} \mathrm{C}$, while the sulfur and selenium injection solution was allowed to cool to room temperature. For each shell growth, a calculated amount of a given precursor solution was injected with a syringe using standard air-free procedures. This was followed by the QD extraction step. ${ }^{[41]}$ Further purification of QDs was done by precipitating them with acetone or methanol. Highly pure QDs were obtained by repeating above purification procedure for 2-3 times. Aliquots of the solution were removed after each monolayer of shell growth with a syringe and tested for optical spectra. Three different QDQWs with increasing QW thickness were synthesized: CdS/1.5 MLs CdSe/3 MLs ZnS, CdS/3 MLs CdSe/3 MLs ZnS and CdS/4.5 MLs CdSe/4 MLs ZnS. The thickness of QWs ranges from $0.35 \mathrm{~nm}$ to $1.6 \mathrm{~nm}$. 

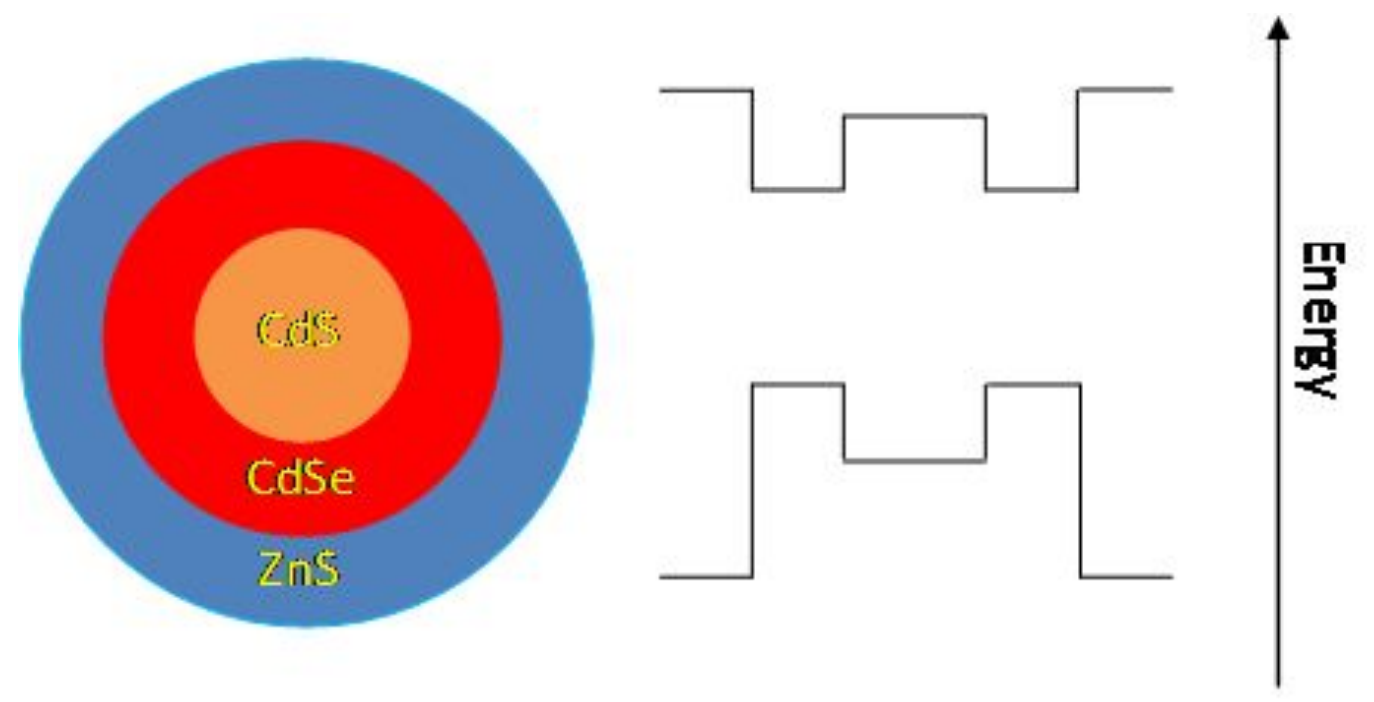

Fig. 3.4 Schematic cross-sections and energy band diagrams of QDQW structures

Figure 3.5 shows the evolution of the absorption spectra of the three different QDQW structures during the shell growth. As the thickness of QW increases, the excitonic peak exhibits red shift and broadening due to the penetration of the electron and/or hole wave functions into the shell layers. The secondary excitonic peak becomes dominant when the CdSe QW grow thicker, indicating that CdSe QW absorption may dominate the absorption process. 

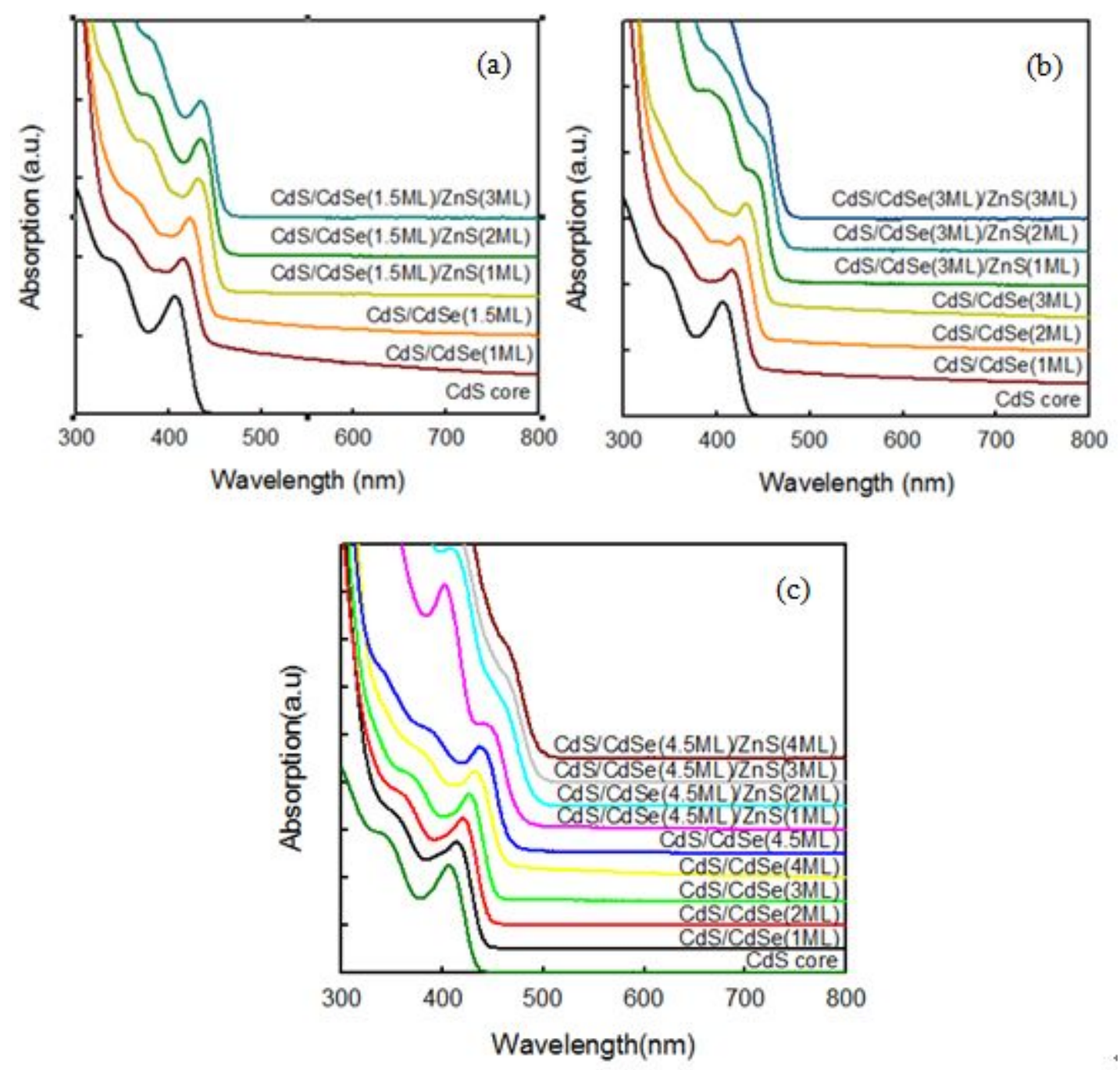

Fig. 3.5 Evolution of the absorption spectra of (a) CdS/CdSe(1.5 MLs)/ZnS(3 MLs) (b) CdS/CdSe(3 MLs)/ZnS(3 MLs) (c) CdS/CdSe(4.5 MLs)/ZnS(4 MLs) QDs during the shell growth.

Figure 3.6 shows the photoluminescence (PL) spectra of the three different QDQW structures during the shell growth. The broad emission in CdS core QDs were largely suppressed after $1 \mathrm{ML}$ of CdSe growth. Only CdS core emission is seen when 1.5 MLs CdSe QW is grown on CdS core QD. However, as the CdSe QW grows thicker, the PL spectra show quite different phenomenon from the 1.5 MLs CdSe. After the overgrowth of $\mathrm{ZnS}$ layers, both of their PL spectra split into two peaks, corresponding to the CdS core peak CdSe QW peak, respectively. The outer ZnS shell and inner CdS core act as cladding layers leading to the formation of a CdSe QW. The QW emission peaks at $467 \mathrm{~nm}$ (3 MLs) and $500 \mathrm{~nm}$ (4.5 MLs), with a FWHM of $~ 30$ $\mathrm{nm}$, whereas the $433 \mathrm{~nm}$ peak arises from the CdS core. 

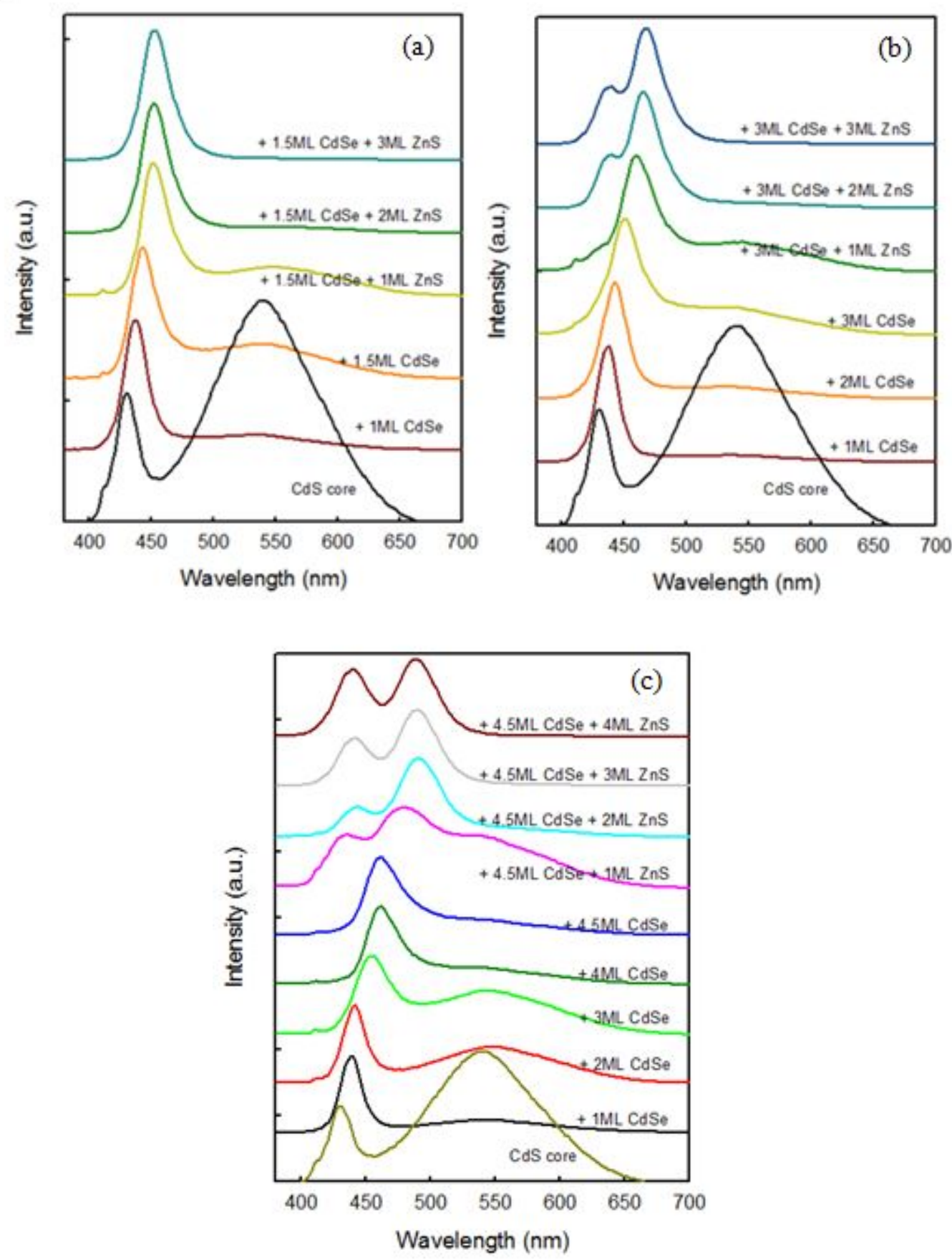

Fig. 3.6 Evolution of the PL spectra of (a) CdS/CdSe(1.5 MLs)/ZnS(3 MLs) (b) $\mathrm{CdS} / \mathrm{CdSe}(3$ MLs)/ZnS(3 MLs) (c) CdS/CdSe(4.5 MLs)/ZnS(4 MLs) QDs during the shell growth.

The evolution of the PL QY of the QDQWs in solutions has been investigated (Figure. 3.7). The QY of the CdS core QDs was only 2.1\%, while overgrowth of 1 ML of CdSe shell raised the QY to $14.6 \%$, but decreased as the CdSe shell grew thicker. This behavior has been observed in many core/shell QDs with a significant lattice mismatch between the core and shell. ${ }^{[54]}$ The initial shell growth is coherent and 
strain builds up in the shell as it grows thicker. When a critical thickness is reached, strain is relieved through the formation of misfit dislocations at the interface, which act as nonradiative recombination sites. The QY was remarkably improved after overcoating the QDs with a ZnS shell. Clearly, the wide bandgap ZnS shell effectively confines carriers in the QW and passivates the nanocrystal surface. A maximum value of $\sim 46 \%$ after the growth of $3 \mathrm{MLs}$ of $\mathrm{ZnS}$ was obtained. Given that the CdS core emission accounts for $\sim 25 \%$ of the total luminescence, the QY of the CdSe QW PL is $\sim 35 \%$. Such high efficiency blue light emission from CdSe nanocrystals has not been reported before.

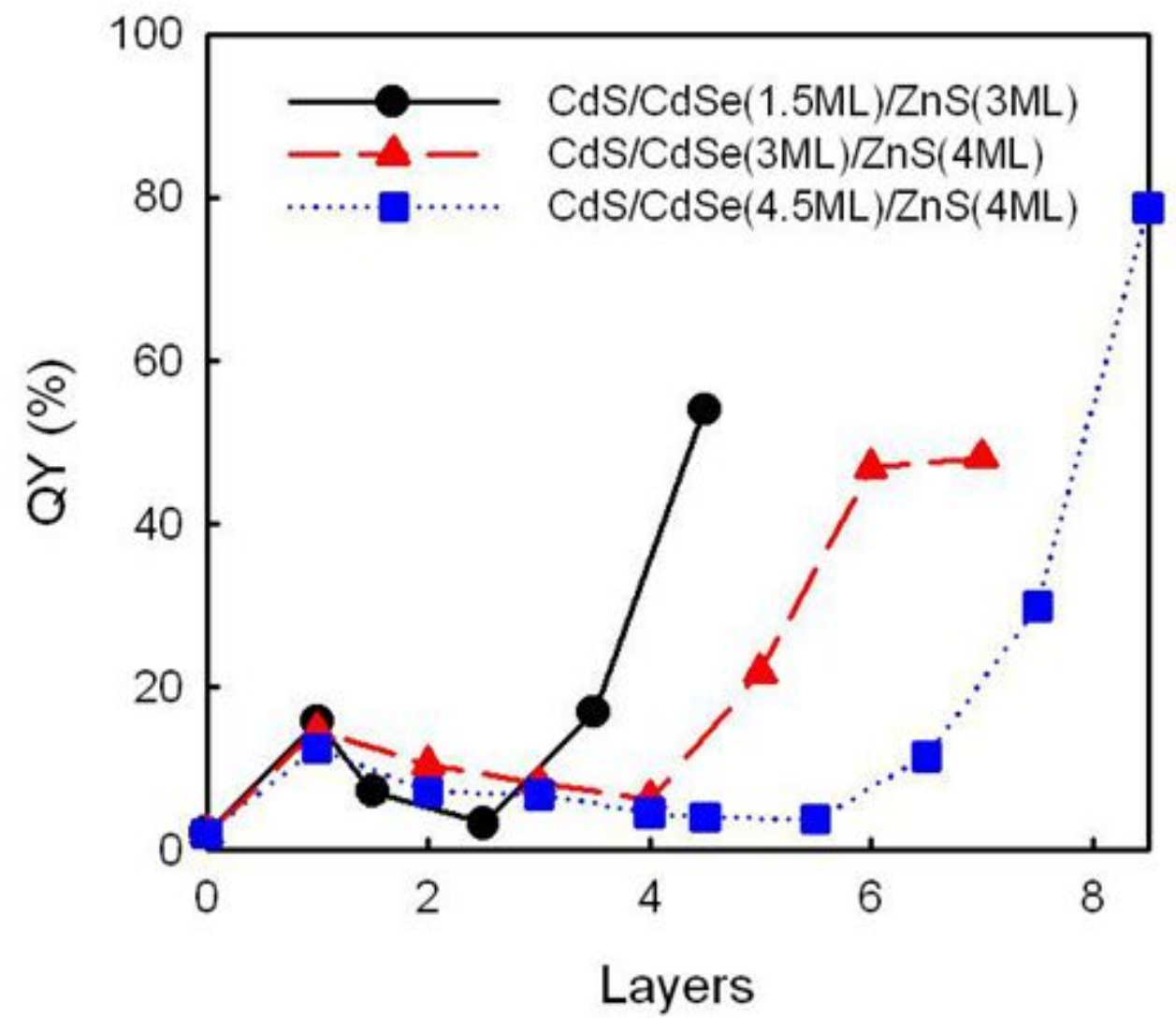

Fig. 3.7 Evolution of the PL QY based on different QW thickness

The crystallinity of the CSS QDs was examined by powder X-ray diffraction (XRD) on a Bruker D8 Discover X-Ray Diffractometer using $\mathrm{Cu}$ a radiation ( $\mathrm{nm}$ ). Figure 3.8 shows the representative XRD pattern of CdS/CdSe (3 MLs)/ZnS (2 MLs) QDQW. Three distinct diffraction peaks were observed at $2 \mathrm{~h}$ values of 26.8, 44.2, and 51.2 degree, corresponding to the (111), (220), and (311) crystalline planes of 
zinc-blende CdS, respectively. The broadening of the diffraction peaks is expected due to the nanocrystalline nature of the particles.

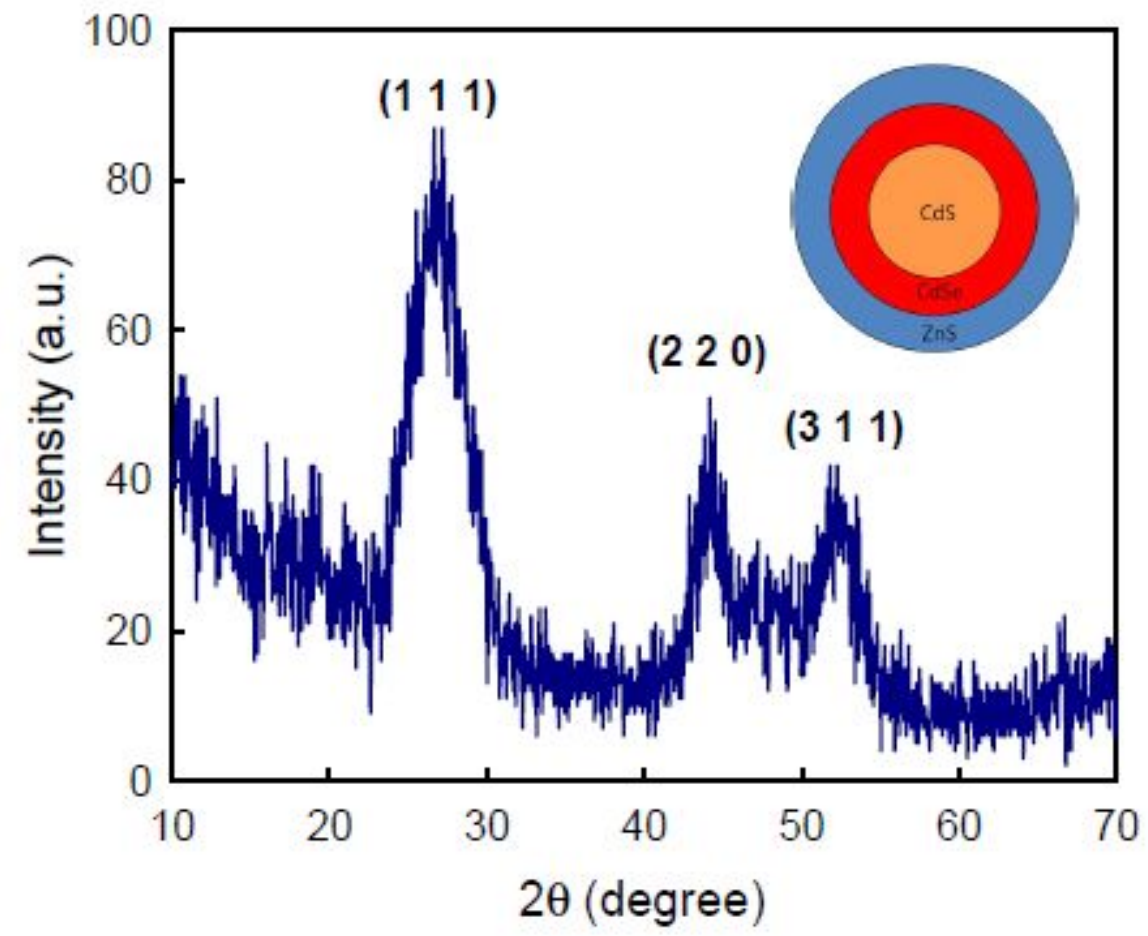

Fig. 3.8 Powder XRD of CdS/CdSe (3 MLs)/ZnS (2 MLs) QDQWs.

\subsection{Demonstration of Blue-Emitting QDQW-LEDs}

To achieve blue EL from QDQWs, the CdS/CdSe (3 MLs)/ZnS (3 MLs) QDQW NCs synthesized above were further incorporated into a LED structure as the light-emitting layer (EML). The QDQW-LEDs were fabricated on glass substrates with pre-patterned indium-tin-oxide (ITO) (sheet resistance $\sim 15 \Omega / \square$ ). The device structure consisted of a $40 \mathrm{~nm}$ PEDOT:PSS HIL, a $40 \mathrm{~nm}$ poly-TPD HTL, a QDQW EML, and a $40 \mathrm{~nm}$ TPBi ETL. The PEDOT:PSS, Poly-TPD, and QDQW layers were prepared using spin-coating. As shown in Figure. 3.9 (a), the QDQW EML was deposited either by spin coating from a $3 \mathrm{mg} / \mathrm{ml} \mathrm{QD} / \mathrm{h}$ exane solution, which resulted in $\sim 3$ MLs of closely-packed QDQWs, or by spin coating of a mixture of QDQWs (3 $\mathrm{mg} / \mathrm{ml}$ ) and poly(N-vinylcarbazole) (PVK) $(3 \mathrm{mg} / \mathrm{ml}$ in chloroform), forming a $\sim 30$ nm-thick hybrid layer with QDQWs embedded in a PVK matrix. Figure 3.9 (b) display the energy diagram of the QDQW-LEDs. Finally, LiF/Al $(0.5 / 120 \mathrm{~nm})$ was 
deposited on top of the TPBi layer as the cathode, whose overlap with the ITO anode defines an emitting area of $0.1 \mathrm{~cm}^{2}$.

(a)
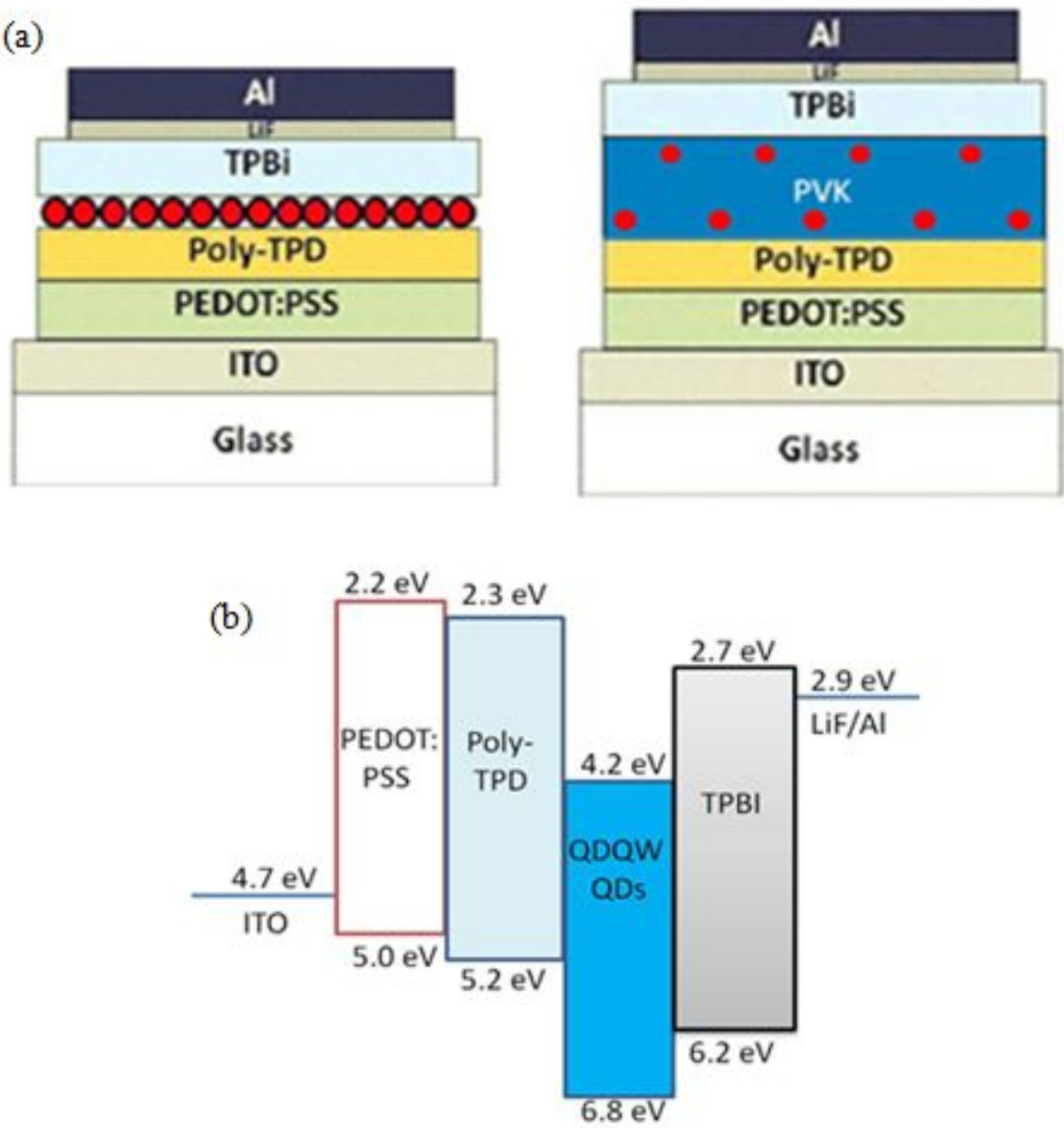

Fig. 3.9 (a) Layer structure of two different structures of QDQW-LED: closed packed QDQW (left) and QDQW embedded in a PVK matrix (right) (b) The energy diagram of the QDQW-LED

The QD-LEDs were encapsulated then with glass lids in $\mathrm{N}_{2}$ and characterized in air at room temperature. The electrical characteristics were measured using an Agilent 4156C semiconductor analyzer. The EL spectra were recorded as a function of injection current density in the range of $1-50 \mathrm{~mA} / \mathrm{cm}^{2}$ using a silicon photodiode-array fiber-optic spectrometer. To calculate EQE, the QD-LEDs were placed directly on the surface of a calibrated silicon photodetector and all emitted photons from the glass side were captured.

The current-voltage (I-V) characteristics of the QDQW-LEDs with different EMLs are shown in Figure 3.10. Both devices turn on at $\sim 9 \mathrm{~V}$ and have similar series 
resistances. Their operational voltages at $20 \mathrm{~mA} / \mathrm{cm}^{2}$ are $13.2 \mathrm{~V}$ and $13.4 \mathrm{~V}$, respectively. It is believed that the voltage largely drops at the interfaces between the QDQW EML and charge transport layers, where, injected electrons and holes face large energy barriers due to large energy band offsets. The resulting poor charge injection into the QDQWs may be a major factor limiting the performance of the LEDs.

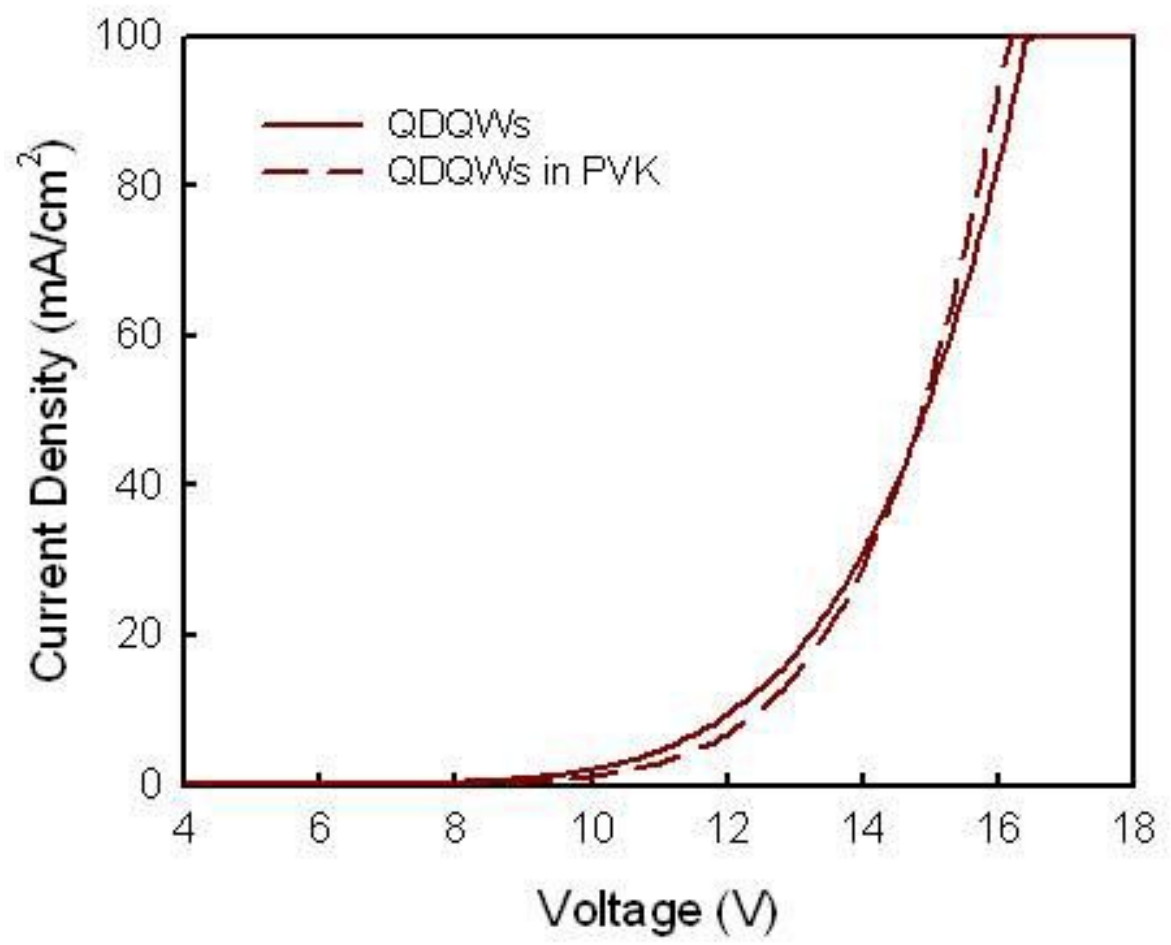

Fig. 3.10 I-V characteristics of LEDs with an EML comprising closely-packed CdS/CdSe/ZnS QDQWs or QDQWs embedded in a PVK host matrix. 

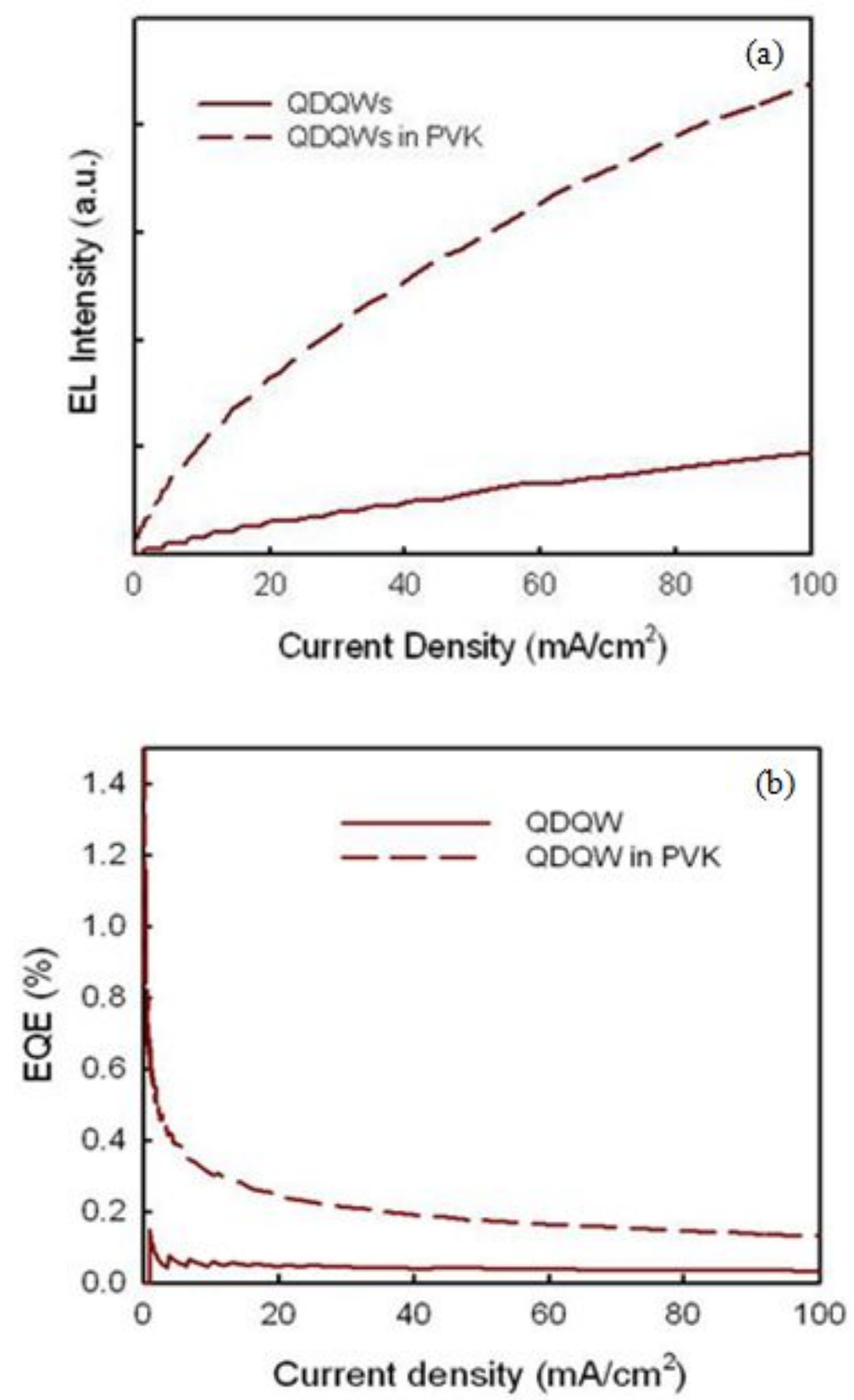

Fig. 3.11 (a)

characteristics of QDQW-LEDs (b) EQE of QDQW-LEDs with an EML comprising closely-packed CdS/ CdSe/ZnS QDQWs or QDQWs embedded in a PVK host matrix.

Figure 3.11 (a) shows the luminance-current (L-I) characteristics of the two QDQW-LEDs. The LED with QDQWs in PVK is five times brighter than the LED with closely-packed QDQWs. This can been attributed to more efficient charge injection and energy transfer from the PVK host surrounding the QDQWs in the former device. However, both LEDs are not efficient under current injection. Figure 3.11 (b) shows that the EQE of the two LEDs. At $20 \mathrm{~mA} / \mathrm{cm}^{2}$, their EQEs are only 
$0.24 \%$ and $0.05 \%$. These values are much lower than the PL QYs of the QDQWs measured in solution. This discrepancy can mainly be attributed to (i) detachment of ligands from the surface of the $\mathrm{ZnS}$ cladding layer during device processing, leading to degraded nanocrystal quality, and (ii) poor charge injection into the QDQWs, limiting the exciton generation rate.

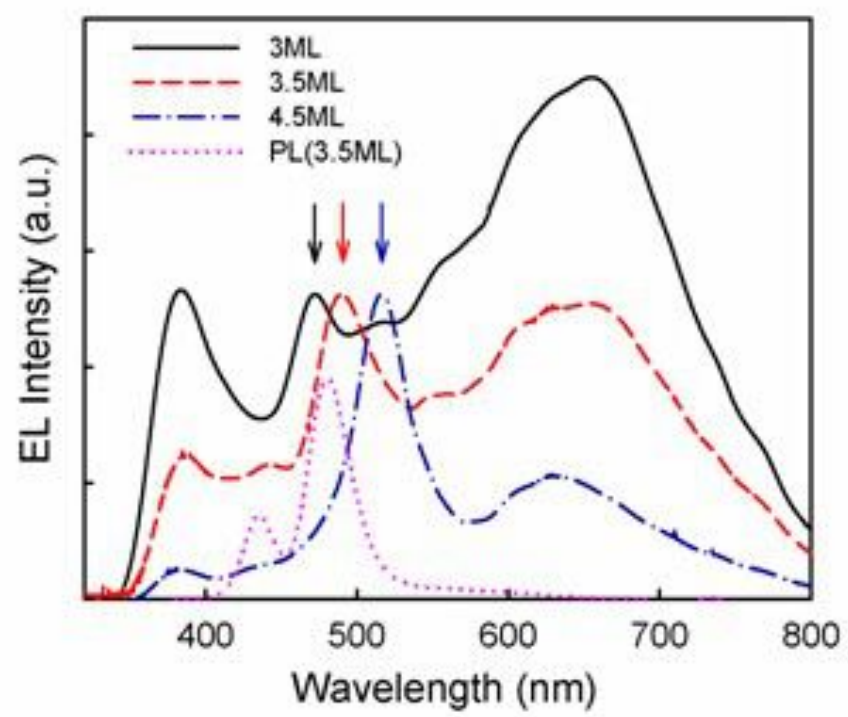

Fig. 3.12 EL spectra of QDQW-LEDs with an EML comprising CdS/CdSe(3, 3.5, 4.5 MLs)/ZnS(3 MLs) QDQWs measured at $10 \mathrm{~mA} / \mathrm{cm}^{2}$. The arrows indicate the QW emission peaks whose intensities were normalized. The PL spectrum of the CdS/CdSe(3.5 MLs)/ZnS(3 MLs)QDQWs measured in hexane is also shown.

Figure 3.12 illustrates the EL spectra of QDQW-LEDs with a $3 \mathrm{ML}, 3.5 \mathrm{ML}$ or 4.5 ML CdSe QW recorded at $10 \mathrm{~mA} / \mathrm{cm}^{2}$. The respective QW emission peaks are located at $472 \mathrm{~nm}, 489 \mathrm{~nm}$ and $516 \mathrm{~nm}$, and their intensity ratio is 1:2.1:2.9. These peaks exhibit a small red shift with respect to their respective PL peaks measured in solution, as seen from the example of 3.5 ML sample. The EL spectra of all the LEDs also include a small UV peak at $\sim 385 \mathrm{~nm}$ originating from TPBi and a long-wavelength broadband extending beyond $800 \mathrm{~nm}$, whereas the emission from the CdS core at $\sim 442 \mathrm{~nm}$ can barely be seen. The presence of the organic peak suggests that, despite the large HOMO of TPBi $(\sim 6.2 \mathrm{eV})$, some excitons can still form on its 
molecules due to hole overinjection. The broadband emission mainly arises from carrier recombination via defects at the $\mathrm{ZnS}$ shell surface and the $\mathrm{CdS} / \mathrm{CdSe}$ interface. Some of these defects are also responsible for the long-wavelength PL band (Figure 3.6), but is less noticeable there due to much more efficient luminescence from the $\mathrm{CdS}$ core. In contrast to dominant QW emission under optical excitation, the pronounced defect emission under electrical excitation confirms the above conclusion that the EL efficiency is largely limited by poor charge injection into the QDQWs. As the QW width decreases, the bandgap increases and the QW emission shifts toward the shorter wavelength. It can be expected that charge injection faces an increasingly large barrier. Indeed, the blue QDQW-LED has the lowest EL intensity among the three LEDs, and as seen from Figure 3.12, its EL spectrum is dominated by defect emission to the largest degree. Meanwhile, its EL also has the strongest organic fluorescence as more holes are overinjected and recombined with electrons in the TPBi layer. Further work will focus on surface modification of the QDQW nanocrystals for energy barrier reduction so as to achieve more facile hole injection.

As compared with green LEDs based on $\mathrm{CdSe} / \mathrm{ZnS}$ core/shell QDs fabricated in our lab, the green QDQW-LED is one order of the magnitude less efficient. Hence, its poor performance can only in part be attributed to poor charge injection. Another possible factor playing a significant role is the quantum-confined Stark effect (QCSE). It is well known that the QCSE has a considerable impact on the optical properties of planar QWs, causing a red shift of optical transitions and a strong reduction in the oscillator strength. ${ }^{[62]}$ In 0D QDs, large field-induced Stark shifts of the energy levels of spatially confined excitons have been observed, but the effect is not accompanied by a dramatic decrease in the oscillator strength of optical transitions. ${ }^{[62,63]}$ It is believed that this behavior is a result of strong quantum and spatial confinement of electrons and holes in QDs. However, the QCSE in QDQWs is expected to have a much stronger impact due to two processes occurring under an electric field: (i) field-assisted tunneling of electrons and holes from the QW layer into the QD core or defect states, and (ii) field-driven spatial separation of electrons and holes in the QW layer. The latter should be facilitated by the circular geometry of nanocrystal QWs as 
compared to conventional planar QWs, pushing electrons and holes toward two sides of the QDQWs. So it is possible to argue that the unique QDQW heterostructure might lead to a stronger QCSE, weakening the oscillator strength of optical transitions.

\subsection{Conclusions}

In summary, colloidal CdS/CdSe/ZnS QDQW nanocrystals were synthesized and characterized. Blue and green luminescence has been achieved by tuning the CdSe QW width in the range of 3-4.5 MLs $(\sim 1.05-1.58 \mathrm{~nm})$, and enhanced through bandgap and strain engineering. The PL of CdS/CdSe (3 MLs)/ZnS (3 MLs) QDQWs peaked at $\sim 467 \mathrm{~nm}$ and had a QY 35\%. The EL spectra of QDQW-LEDs with 3-4.5 ML CdSe consisted of QW emission ranging from 472-516 $\mathrm{nm}$ and a visible broadband emission from defect states. The overall EQE was only a few tenth percent, which was limited by poor and unbalanced charge injection into the QDQW nanocrystals. The LEDs may also suffer from a strong QCSE resulting from the unique circular geometry of the nanocrytsal QWs, which weakens the oscillator strength of optical transitions. 


\section{Chapter 4 Conclusions and Future Work}

\subsection{Conclusions}

This thesis work has focused on the synthesis and characterization of two types of $\mathrm{CdZnS}(\mathrm{Se})$-based colloidal nanocrystal heterostructures, i.e. strain-compensated core/multishell CdSe/ZnS/ZnCdS QDs and CdS/CdSe/ZnS QDQWs, and explored their application in LED devices as high-efficiency luminescence. The primary findings of the research are summarized as the following:

1. Synthesis of CdSe QDs with strain compensated shell for high-efficiency light emission (Chapter 2)

- $\mathrm{CdSe} / \mathrm{ZnCdS} / \mathrm{ZnS}$ and CdSe/ZnS/ZnCdS core/shell/shell (CSS) QDs were synthesized and characterized. The synthesis process included growth of the core QDs by the hot injection technique and growth of the multishell by the SILAR method.

- A ZnCdS strain-compensating outer shell has proven to be more effective than a $\mathrm{ZnCdS}$ strain-reducing intermediate shell in improving the PL QY of CdSe/ZnS QDs. The resulting CdSe/ZnS/ZnCdS CSS QDs were 40\% more efficient than the $\mathrm{CdSe} / \mathrm{ZnCdS} / \mathrm{ZnS}$ QDs, and have a more controllable peak wavelength.

- QD-LEDs based on the CSS QDs were designed and fabricated by integrating the QD emission layer into the ITO/PEDOT:PSS/poly-TPD/QDs/TPBI /LiF/Al structure. The LEDs with strain-compensated CSS QDs exhibited 28\% higher brightness and purer EL spectra. These results suggest that nanocrystal QDs of high crystalline and optical quality can be obtained through strain-engineering in their nanoshell structures.

2. Synthesis of CdSe Quantum-Dot Quantum-Well nanocrystals for blue light emission (Chapter 3) 
- High-quality colloidal CdS QDs with sharp excitonic absorption peaks were synthesized by the hot-injection method. Their PL spectra, however, are dominated by long-wavelngth surface state emission.

- $\quad \mathrm{CdS} / \mathrm{CdSe} / \mathrm{ZnS}$ QDQW nanocrystals were synthesized by growing a $\mathrm{CdSe} / \mathrm{ZnS}$ shell over the CdS core using the SILAR method. Efficient blue PL emission peaking at $\sim 467 \mathrm{~nm}$ was obtained from a $3 \mathrm{MLs}$ CdSe nanocrystal QW ( 1.05 nm) with a QY $\sim 35 \%$.

- QDQW-LEDs with a closely-packed QDQW emitting layer or an emitting layer comprising QDQWs embedded in a PVK host were successfully demonstrated. The latter structure benefited from excitonic energy transfer from the host and showed 5x more efficient EL.

- Blue and green (472-516 nm) EL was obtained from QDQW-LEDs with 3-4.5 ML CdSe QWs. It was found that as the QW peak blue shifted, the overall EL was increasingly dominated by defect state emission due to poor hole injection into the QDQWs.

\subsection{Future Work}

Since direct injection of carriers into QDs is very difficult due to their low valance band energies, exciton energy transfer plays an important role in the EL of QDQW-LEDs. Moreover, under current injection, only a small number of excitons are generated in the QDQWs due to poor charge injection. Also, a large portion of those excitons maybe trapped at the defect states at the $\mathrm{CdS} / \mathrm{CdSe}$ interface, contributing to broadband emission, and thus, degrade the EQE.

In order to realize high efficiency color-saturated blue nanocrystal QDQW-LEDs, further work toward performance enhancement should be focused on the following two aspects:

(1) The QDQW synthesis needs to be further optimized to eliminate CdS core emission and surface/interface state emission. It is expected that the core emission can be removed by using a smaller CdS core or inserting a wide bandgap interlayer as the 
exciton blocking layer. Defect emission can be suppressed by improving the quality and optimizing the thickness of the outer shell.

(2) The QDQW-LEDs layer structure used in this study was not optimized to realize the maximal exciton generation and energy transfer. . An ideal structure would enable efficient excitonic energy transfer from the organic charge transport layers to the QDQW emitting layer. This would overcome the problem of poor charge injection into the QDQWs.

Finally, the QDQW-LEDs may also suffer from a strong quantum-confined Stark effect (QCSE) resulting from the unique circular geometry of the nanocrytsal QWs, which weakens the oscillator strength of optical transitions. This hypothesis needs to be verified via a proper design of testing experiments. 


\section{Appendix}

\section{A.1 Synthesis of CdSe/ZnS Core/Shell Quantum Dots with 6 MLs of ZnS Shell}

A.1.1 Preparation of the Precursor Solution for the SILAR Method

For shell growth using the SILAR method, ${ }^{[18]}$ we used precursor solutions containing only metal oxides $(\mathrm{ZnO})$, and elemental sulfur. The typical zinc precursor solution $(0.05 \mathrm{M})$ was prepared by dissolving $\mathrm{ZnO}(81.0 \mathrm{mg})$ in $\mathrm{OA}(2.54 \mathrm{~mL})$ and ODE $(17.46 \mathrm{~mL})$ at $310^{\circ} \mathrm{C}$. The sulfur precursor solution $(0.05 \mathrm{M})$ was prepared in the similar way by dissolving sulfur $(32.0 \mathrm{mg})$ in $\mathrm{ODE}(20.0 \mathrm{~mL})$ at $180{ }^{\circ} \mathrm{C}$. All of the solutions were freshly prepared under $\mathrm{N}_{2}$ atmosphere. The Zn-precursor solutions were kept at about $100{ }^{\circ} \mathrm{C}$, while the sulfur injection solution was allowed to cool to room temperature. For each shell growth, a calculated amount of a given precursor solution was injected with a syringe using standard air-free procedures.

\section{A.1.2 Calculations of the Amount of Precursor Solutions for Shell Growth}

The growth of CdSe/ZnS core/shell QDs using the SILAR method is based on alternating injections of the $\mathrm{Zn}$ - and S-precursors into the solution containing the CdSe core QDs. The concentration of CdSe core QDs can be estimated on the basis of the absorption spectra. ${ }^{[53]}$ The amount of the injection solution for each monolayer can be deduced from a calculation of the number of surface atoms:
The size of CdSe core:
$\mathrm{D}_{0}=2.64 \mathrm{~nm}$
$\mathrm{R}_{0}=1.32 \mathrm{~nm}$

The molar quantity of CdSe core QDs: $\mathrm{n}_{1}=$

The average thickness of one monolayer of $\mathrm{ZnS}(\mathbf{d}): \quad d=0.31 \mathrm{~nm}$

The amount of $\mathrm{Zn}$ and $\mathrm{S}$ precursor of the first layer $\mathrm{ZnS}$ (1 ML):

The volume of the first $\mathrm{ZnS}$ monolayer: $\mathrm{V}_{1}=4 \pi\left(\mathrm{R}_{1}{ }^{3}-\mathrm{R}_{0}{ }^{3}\right) / 3$

$$
\mathrm{R}_{0}=1.32 \mathrm{~nm} \quad \mathrm{R}_{1}=1.32+0.31=1.63 \mathrm{~nm}
$$




$$
\mathrm{V}_{1}=4 \times 3.14 \times\left(1.63^{3}-1.32^{3}\right) / 3=8.502 \mathrm{~nm}^{3}=8.502 \times 10^{-27} \mathrm{~m}^{3}
$$

The density of $\mathrm{ZnS}$ material: $\quad \mathrm{D}=4.11 \times 10^{3} \mathrm{~kg} / \mathrm{m}^{3}$

The mass of the first ML ZnS shell for a single core/shell QD:

$$
\begin{gathered}
\mathrm{m}=\text { Volume } \times \text { Density } \\
\mathrm{m}=8.502 \times 10^{-27} \mathrm{~m}^{3} \times 4.11 \times 10^{3} \mathrm{~kg} / \mathrm{m}^{3}=3.494 \times 10^{-23} \mathrm{~kg}
\end{gathered}
$$

The molar quantity of $\mathrm{ZnS}$ for a single core/shell QD: $n=m / M . W$.

$$
\begin{gathered}
\text { M.W. }(\text { Molecular Weight })=9.746 \times 10^{-2} \mathrm{~kg} / \mathrm{mol} \\
\mathrm{n}_{2}=\mathrm{m} / \mathrm{M} . \mathrm{W} .=3.494 \times 10^{-23} \mathrm{~kg} / 9.746 \times 10^{-2} \mathrm{~kg} / \mathrm{mol}=3.585 \times 10^{-22} \mathrm{~mol} / \mathrm{QD}
\end{gathered}
$$

The molar quantity of $\mathrm{Zn}-/ \mathrm{S}-$ precursor for the first ML ZnS growth:

$$
\begin{gathered}
\mathrm{A}_{1}=\mathrm{n}_{1} \times \mathrm{N} \times \mathrm{n}_{2} \\
\mathrm{~A}_{1}=1 \times 10^{-6} \mathrm{~mol} \times\left(6.02 \times 10^{23} \mathrm{QD} / \mathrm{mol}\right) \times 3.585 \times 10^{-22} \mathrm{~mol} / \mathrm{QD}=2.158 \times 10^{-4} \mathrm{~mol} \\
=\mathbf{0 . 2 1 6} \mathbf{~ m m o l}
\end{gathered}
$$

2ML ZnS: $\mathrm{A}_{2}=\mathrm{A}_{1} \times \mathrm{V}_{2} / \mathrm{V}_{1}=0.163 \times\left(1.94^{3}-1.63^{3}\right) /\left(1.63^{3}-1.32^{3}\right)=\mathbf{0 . 3 0 8} \mathbf{~ m m o l}$

3ML ZnS: $\mathrm{A}_{3}=\mathrm{A}_{1} \times \mathrm{V}_{3} / \mathrm{V}_{1}=0.163 \times\left(2.25^{3}-1.94^{3}\right) /\left(1.63^{3}-1.32^{3}\right)=\mathbf{0 . 4 2 4} \mathbf{~ m m o l}$

4ML ZnS: $\mathrm{A}_{4}=\mathrm{A}_{1} \times \mathrm{V}_{4} / \mathrm{V}_{1}=0.163 \times\left(2.56^{3}-2.25^{3}\right) /\left(1.63^{3}-1.32^{3}\right)=\mathbf{0 . 5 5 8} \mathbf{~ m m o l}$

5ML ZnS: $\mathrm{A}_{5}=\mathrm{A}_{1} \times \mathrm{V}_{5} / \mathrm{V}_{1}=0.163 \times\left(2.87^{3}-2.56^{3}\right) /\left(1.63^{3}-1.32^{3}\right)=\mathbf{0 . 7 1 1} \mathbf{~ m m o l}$

5ML ZnS: $\mathrm{A}_{6}=\mathrm{A}_{1} \times \mathrm{V}_{6} / \mathrm{V}_{1}=0.163 \times\left(3.18^{3}-2.87^{3}\right) /\left(1.63^{3}-1.32^{3}\right)=\mathbf{0 . 8 8 2} \mathbf{~ m m o l}$

For the actual CdSe/ZnS core/shell QDs growth, we assumed that the surface of the CdSe core consists equally of Se- and Cd- atoms, and therefore used only $50 \%$ of the molar quantity of calculated $\mathrm{Zn}$-/S- precursor for a complete monolayer. ${ }^{[20]}$

\section{A.1.3 Synthesis of CdSe/ZnS Core/Shell QDs Using the SILAR Method}

A typical SILAR synthesis was performed as follows: $18 \mathrm{~mL}$ of ODE and $6 \mathrm{~g}$ of ODA were loaded into in a three-necked flask, heated to $100{ }^{\circ} \mathrm{C}$ under vacuum for $30 \mathrm{~min}$, and cooled to room temperature. The CdSe core QDs in hexane (2.64 $\mathrm{nm}$ in diameter, ) were added, and the system was kept at $100{ }^{\circ} \mathrm{C}$ under vacuum for $30 \mathrm{~min}$ to remove the hexane and other undesired materials of low vapor pressure. Subsequently, the solution was heated to $240{ }^{\circ} \mathrm{C}$ under $\mathrm{N}_{2}$ atmosphere where the shell growth was performed. As calculated in section A.1.2, for the "first injection", we 
used only $1.1 \mathrm{~mL}$ instead of $2.2 \mathrm{~mL}$ of the $\mathrm{Zn}$-precursor solution. As schematically shown in Figure 2.11 (a), this is followed by alternating addition of S-precursors and Zn-precursors, respectively, the amount of which was calculated from the respective volumes of concentric spherical shells with a thickness of one hypothetical monolayer. A period of 10 min between each addition was sufficient for the reaction to be completed. The complete coverage procedure of CdSe cores with $6 \mathrm{MLs}$ of $\mathrm{ZnS}$ took about $2 \mathrm{~h}$ in total. After this time, the solution was cooled to room temperature. For purification, hexane was added and the unreacted compounds and byproducts were removed by successive methanol extraction ${ }^{[53]}$ (at least two times) until the methanol phase was clear.

\section{A.2 Characterization of Quantum Yields of Colloidal}

\section{Quantum Dots}

The photoluminescence (PL) quantum yield (QY) is defined as follows:

$\mathrm{QY}=[$ number of PL photons emitted $] /[$ number of incident photons absorbed $]$

Absolute measurements of QY are difficult, but it is straightforward to find the ratio of the QY for two substances, denoted as 1 and 2:

$$
\mathrm{QY}_{2} / \mathrm{QY}_{1}=\left[\mathrm{F}_{2} / \mathrm{A}_{2}\right] /\left[\mathrm{F}_{1} / \mathrm{A}_{1}\right]=\left[\mathrm{F}_{2} / \mathrm{F}_{1}\right] \times\left[\mathrm{A}_{1} / \mathrm{A}_{2}\right]
$$

Where $\mathrm{F}_{1}=$ Integrated intensity of PL spectrum of the substance 1;

$\mathrm{F}_{2}=$ Integrated intensity of PL spectrum of the substance 2;

$\mathrm{A}_{1}=$ Absorbance at wavelength $\lambda_{\mathrm{a}}$ of absorption spectrum of substance 1;

$\mathrm{A}_{2}=$ Absorbance at wavelength $\lambda_{\mathrm{a}}$ of absorption spectrum of substance 2;

The fluorescence spectrophotometer excites PL of the sample with incident light of a known wavelength $\lambda_{\mathrm{a}}$. The total number of PL photons emitted is proportional to the area under the PL intensity vs. wavelength of the PL spectrum. This integral area is the quantity F. The total number of incident photons that are absorbed during the PL process is proportional to the absorbance A at wavelength $\lambda_{\mathrm{a}}$, which can be measured by the UV-Vis absorption spectrometer. For best results in determining QYs, the two substances should have absorption peaks at approximately the same wavelengths, and their PL spectra should overlap as much as possible. 


\section{Reference:}

[1] C. B. Murray, D. J. Norris, M. G. Bawendi , J. Am. Chem. Soc. 1993, 115 , 8706 .

[2] M. G. Bawendi, M. L. Steigerwald, L. E. Brus, Annu. Rev. Phys. Chem. 1990, 41, 477.

[3] A. P. Alivisatos, A. L. Harris, N. J. Levinos, M. L. Steigerwald, L. E. Brus, J.

Chem. Phys. 1988, 89, 4001.

[4] W. Luan, H. Yang, N. Fan, S. Tu, Nanoscale Res Lett. 2008, 3(4), 134-139

[5] L. Esaki, R. Tsu, IBM Journal of Research and Development 1970, 14, 61-65.

[6] D. Bera, L. Qian, T. K. Tseng and P. H. Holloway, Materials 2010, 3, 2260-2345.

[7] V. Klimov, Los Alamos Science 2003, 28, 214-220.

[8] M. Bruchez, M. Moronne, P. Gin, S. Weiss, A. P. Alivisatos, Science 1998, 281, 2013.

[9] A. L. Rogach, N. Gaponik, J. M. Lupton, C. Bertoni, D. E. Gallardo, S. Dunn, N. Li Pira, M. Paderi, P. Repetto, S. G. Romanov, Angewandte Chemie International Edition 2008, 47, 6538-6549.

[10] D. V. Talapin, J. S. Lee, M. V. Kovalenko, E. V. Shevchenko, Chemical reviews 2010, 110, 389-458.

[11] P. Reiss, M. Protie're, and L. Li, Small 2009, 5, No. 2, 154-168

[12] D. Dorfs, A. Eychmu“1ler, Z. Phys. Chem. 2006, 220, 1539-1552.

[13] J. Van Emden, J. Jasieniak, D. E. Gomez, P. Mulvaney, M. Giersig, Aust.J. Chem. 2007, 60, 457-471.

[14] M. A. Hines, P. Guyot-Sionnest, J. Phys. Chem.1996, 100, 468-471.

[15] B. O. Dabbousi, J. RodriguezViejo, F. V. Mikulec, J. R. Heine, H. Mattoussi, R.

Ober, K. F. Jensen, M. G. Bawendi, J. Phys. Chem.B 1997, 101, 9463-9475.

[16] X. G. Peng, M. C. Schlamp, A. V. Kadavanich, A. P. Alivisatos, J. Am. Chem. Soc. 1997, 119, 7019-7029.

[17] X. B. Chen, Y. B. Lou, A. C. Samia, C. Burda, Nano Lett. 2003, 3, 799-803.

[18] J. J. Li, Y. A. Wang, W. Z. Guo, J. C. Keay, T. D. Mishima, M. B. Johnson, X. G. Peng, J. Am. Chem. Soc. 2003, 125, 12567-12575.

[19] D. V. Talapin, I. Mekis, S. Gotzinger, A. Kornowski, O. Benson, H. Weller, J. 
Phys. Chem. B 2004, 108, 18826-18831.

[20] R. G. Xie, U. Kolb, J. X. Li, T. Basche', A. Mews, J. Am. Chem. Soc. 2005, 127, $7480-7488$.

[21] M. Braun, C. Burda, M. A. El-Sayed, J. Phys. Chem. A 2001, 105, 5548-5551.

[22] H. Borchert, D. Dorfs, C. McGinley, S. Adam, T. Moller, H. Weller, A.

Eychmu"ller, J. Phys. Chem. B 2003, 107, 7486-7491.

[23] R. B. Little, M. A. El-Sayed, G. W. Bryant, S. Burke, J. Chem. Phys. 2001, 114, $1813-1822$.

[24] D. Dorfs, H. Henschel, J. Kolny, and A. Eychmu1ller*, J. Phys. Chem. B 2004, $108,1578-1583$.

[25] D. Battaglia, B. Blackman, X. G. Peng, J. Am. Chem. Soc. 2005, 127, 10889-10897.

[26] S. Sapra, S. Mayilo, T. A. Klar, A. L. Rogach, J. Feldmann, Adv. Mater. 2007, 19, $569-571$.

[27] E. A. Dias, S. L. Sewall, P. Kambhampati, J. Phys. Chem. C 2007, 111, 708-713. [28] X. Wang, G. I. Koleilat, J. Tang, H. Liu, I. J. Kramer, R. Debnath, L. Brzozowski, D. A. R. Barkhouse, L. Levina, S. Hoogland, E. H. Sargent, Nat. Photonics 2011, 5, 480.

[29] J. M. Caruge, J. E. Halpert, V. Wood, V. Bulovic', M. G. Bawendi, Nat.

Photonics 2008, 2, 247.

[30] X. Gong, M. Tong, Y. Xia, W. Cai, J. S. Moon, Y. Cao, G. Yu, C-L Shieh, B.

Nilsson, A. J. Heeger, Science, 2009, 325, 1665-1667.

[31] M. V. Kovalenko, B. Spokoyny, J. S. Lee, M. Scheele, A. Weber, S. Perera, D.

Landry, D. V. Talapin, J. Am. Chem. Soc. 2010, 132, 6686.

[32] S. Coe-Sullivan, Nat. Photonics 2009, 3, 315-316.

[33] J. S. Steckel, S. Coe-Sullivan, V. Bulovic, M.G. Bawendi, Adv. Mater. 2003, 15, $1862-1866$.

[34] V. L. Colvin, M. C. Schlamp, A.P. Alivisatos, Nature 1994, 370: 354 7.

[35] M. Schlamp, X. Peng, A. Alivisatos, Journal of applied physics 1997, 82, 5837.

[36] S. Coe, W. Woo, M. G. Bawendi, V. Bulovic, Nature 2002, 420, 800-803. 
[37] V. Wood and V. Bulovic', Nano Rev. 2010, 8, 5202

[38] P. O. Anikeeva, J. E. Halpert, M. G. Bawendi, V. Bulovic', Nano Lett 2009, 9, 2532_6.

[39] L. A. Kim LA, P. O. Anikeeva, S. Coe-Sullivan, J. S. Steckel, M. G. Bawendi, V. Bulovic', Nano Lett 2008, 8, 4513_7.

[40] P. O. Anikeeva, J. E. Halpert, M. G. Bawendi, V. Bulovic', Nano Lett 2007, 7, 2196_200.

[41] T. H. Kim, K. S. Cho, E. K. Lee, S. J. Lee, J. Chae, J. W. Kim, D. H. Kim, J. Y. Kwon, G. Amaratunga, Nature Photonics 2011, 5(3), 176-182.

[42] B. S. Mashford, M. Stevenson, Z. Popovic, C. Hamilton, Z. Zhou, C. Breen, J. Steckel, V. Bulovic, M. Bawendi, S. Coe-Sullivan, P. T. Kazlas, Nat. Photonics 2013, 7, 407.

[43] A. Zaban, O. I. Mićić, B. A. Gregg, A. J. Nozik, Langmuir 1998, 14, 3153.

[44] A. G. Pattantyus-Abraham, I. J. Kramer, A. R.Barkhouse, X. Wang, G.

Konstantatos, R. Debnath, L. Levina, I. Raabe, M. K. Nazeeruddin, M. Grätzel, E. H. Sargent, ACS Nano 2010, 4, 3374.

[45] S. A. McDonald, G. Konstantatos, S. Zhang, P. W. Cyr, E. J. D. Klem, L. Levina, E. H. Sargent, Nat. Mater. 2005, 4, 138.

[46] J. Tang, H. Liu, D. Zhitomirsky, S. Hoogland, X. Wang, M. Furukawa, L. Levina, E. H. Sargent, Nano Lett. 2012, 12, 4889.

[47] J. M. Luther, M. Law, M. C. Beard, Q. Song, M. O. Reese, R. J. Ellingson, A. J. Nozik, Nano Lett. 2008, 8, 3488.

[48] W. Ma, S. L. Swisher, T. Ewers, J. Engel, V. E. Ferry, H. A. Atwater, A. P. Alivisatos, ACS Nano 2011, 5, 8140.

[49] J. Nelson, Imperial College Press: London, 2003.

[50] C. H. Henry, J. Appl. Phys. 1980, 51, 4494.

[51] I. J. Kramer and E. H. Sargent*, Chem. Rev. 2014, 114(1), 863-882.

[52] P. Maraghechi, A. J. Labelle, A. R. Kirmani, X. Lan, M. M. Adachi, S. M. Thon, S. Hoogland, A. Lee, Z. Ning, A. Fischer, A. Amassian, E. H. Sargent, ACS Nano 2013, 7, 6111 . 
[53] W. W. Yu, L. Qu, W. Guo, X. Peng, Chemistry of Materials 2003, 15, 2854-2860.

[54] P. Reiss, S. Carayon, J. Bleuse, A. Pron, Synthetic Metals 2003, 139 (3), 649-652.

[55] J. McBride, J. Treadway, L. C. Feldman, S. J. Pennycook, S. J. Rosenthal, Nano Lett. 2006, 6 (7), 1496-1501.

[56] Q. Sun, Y. A. Wang, L. S. Li, D. Wang, T. Zhu, J. Xu, C. Yang, Y. Li, Nature Photonics 2007, 1, 717-722.

[57] Y. Q. Zhang, X. A. Cao, Appl. Phys. Lett. 2010, 97, 253115.

[58] Y. Q. Zhang and X. A. Cao, Nanotech. 2012, 23, 275702.

[59] Z. Tan, F. Zhang, T. Zhu, J. Xu, A. Y. Wang, J. D. Dixon, L. Li, Q. Zhang, S. E. Mohney, and J. Ruzyllo, Nano Lett. 2007, 7(12), 3803-3807 .

[60] W. Bae, J. Kwak, J. Lim, D. Lee, M. Nam, C. Lee, S. Lee, Nanotech. 2009, 20 (7), 075202.

[61] A. W. Schill, C. S. Gaddis, W. Qian, M. A. El-Sayed Y. Cai, V. T. Milam, K. H. Sandhage, Nano Lett. 2006, 6(9), 1940-1949.

[62] S.A. Empedocles and M. G. Bawendi, Science 1997, 278, 2114-2117

[63] Z. B. Wang, H. C. Zhang, J. Y. Zhang, H. Su, Y. A. Wang, Chin. Phys. Lett. 2010, 27(12), 127803 